\title{
HYDROFORMYLATION OF GLYCALS
}

\author{
by \\ DEREK ABSON \\ B.Sc., University of Birmingham, 1952 \\ M.Sc.. University of Birmingham, 1961
}

A THESIS SUBMITTED IN PARTIAL FULFILMENT OF THE REQUIREMENTS FOR THE DEGREE OF

DOCTOR OF PHILOSOPHY

In the Department

of

Chemistry

We accept this thesis as conforming to the required standard

September, 1964 
In presenting this thesis in partial fulfilment of the requirements for an advanied degree at the University of British Columbia, I agree that the Library shall make it freely available for reference and study. I further agree that permission for extensive copying of this thesis for scholarly purposes may be granted by the Head of my Department or by his representatives. It is understood that copying or publication of this thesis for finarcial gain shall not be allowed. without my written permission.

Department of Chemistry

The University of British Coivmbia, Vancouver 8 , Canada.

Date 2 March 196:5 


\section{ACKNOWLEDGEMENTS}

I wish to express my gratitude to Dr. A. Rosenthal for his constant interest and encouragement during the course of this research. The assistance of Dr. I. D. Hall in the measurement and interpretation of n.m.r. spectra is gratefully acknowledged. Thanks are also due to Drs. E. von Rudloff and P. A. J. Gorin of the Pralrie Regional Laboratory, N.R.C., Saskatoon, for providing reference samples of certain compounds. 


\section{ABSTRACT}

The reaction of $3,4-d 1-0-a c e t y 1-$ D-xylal with 3 moles of synthesis gas $\left(\mathrm{CO}+2 \mathrm{H}_{2}\right)$ under oxo conditions gave predominantly two isomeric 2,3-di-0-acetyl-1,5-anhydro-4-deoxyhexitols, by addition of a hydroxymethyl group at C-I of the glycal. The structures of the two polyols, obtained by deacetylation of the reaction product and fractionation by paper partition chromatography, were completely established. Formation of a pair of enantiomeric triol ethers by perlodate cleavage and sodium borohydride reduction of each polyol showed that they were 1,5-anhydro-4-deoxyhexitols, having unbranched carbon skeletons, this also being shown by the proton resonance positions and intensities in the n.m.r. spectra of the polyols. One of the enantiomeric triol ethers, having the I-configuration, was prepared from a carbohydrate of known structure, 1,4-anhydro-5-deoxy-D-arabino-hex1tol, thereby establishing the configurations at $\mathrm{C}-5$ of the two isomeric 1,5anhydro-4-deoxy-hexitols. Assignments of the D-arabinoand I-xylo- configurations to the two isomers conflicted with results of Gorin ${ }^{82}$, who had previously assigned the D-arabino- configurations to a 1,5-anhydro-4-deoxy-hexito1 which did not resemble either of our compounds. That these were the D-arabino- and L-xylo- isomers of 1,5-anhydro-4- 
deoxy-hexitol was proved by their conversion into a pair of isomeric 1,5-anhydro-4,6-dideoxy-hexitols which were 1dentical with those obtained by the reaction of 3,4-di-0acetyl-2-deoxy-D-xylopyranosyl chloride with methyl magnesium bromide, both series of reactions allowing no possibility of configurational inversions. The polyol described by Gorin was subsequently shown to be the alternative trans isomer, 1,5-anhydro-4-deoxy-D-xylo-hexitol.

A concurrent study of the structures of the two anhydrodeoxyhexitols was made by nuclear magnetic resonance, and the stereochemistry of the L-xylo- isomer could be assigned from the multiplicities of the C-4 proton signals. The single $\mathrm{C}-4$ proton in the deuterated analogue of the L-xylo- isomer (prepared by reacting 3,4-di-o-acetyl-Dxylal with carbon monoxide and deuterlum) was shown to be equatorial by its resonance position, and its multiplicity on deuterium-hydrogen decoupling, this providing evidence for cis-addition to the double bond of the glycal on hydroformylation.

The oxo reaction of 3,4,6-tri-0-acetyl-D-galactal has been reinvestigated, and found to be entirely analogous to those of other glycals, giving, on deacetylation, a mixture of 2,6-anhydro-3-deoxy-D-galacto- and D-taloheptitols. These were isolated and characterised, and their 
stereochemistry established by correlation with the D-gluco1somer, whose structure has been proved by X-ray analysis.

The reaction of 3,4-di-0-acety1-D-xylal under hydroformylation conditions, leading to the formation of aldehydes rather than alcohols, has been investigated. From the reaction of the glycal with 2 moles of synthesis gas, two isomeric 4,5-di-o-acetyl-2,6-anhydro-3-deoxy-aldehydohexoses were isolated as their crystalline 2,4-dinttrophenylhydrazones. These were Identified by conversion of one of them, 4,5-d1-0-acety1-2,6-anhydro-3-deoxy-aldehydoD-1yxo-hexose, to 1,5-anhydro-4-deoxy-D-arabino-hexitol, whose structure had been established previously..: The two aldehydo-hexoses were also obtained when a mixture of 2,3di-0-acetyl-1,5-anhydro-4-deoxy-D-arabino- and I-xylohexitols were reacted with dimethylsulphoxide and $N_{,} N^{\prime}-$ dicyclohexylcarbodilmide. 
The Oxo Reaction 1

(1) Nature of the Catalyst 4

(11) Mechanism of the Oxo Reaction 5

(111) Effect of Olefin Structure on Hydroformylation

The Glycals

(1) Structure of the GIycals $\quad 16$

(11) Preparation of the Glycals 20

(111) Reactions of the GIFcals. $\quad 23$

The Oxo Reaction of Giycals 30

DISCUSSION

A. Hydroxymethylation of 3,4-d1-0-acetyl-D-xylaI

(1) Reactants and Reaction Conditions

(11) Fractionation and Characterisation of Reaction Products

(11i) Identification of Fractions $I$ and II

(1v) Configurations of Fractions I and II

52

(v) Identities of Polyols $X$ and $Y$

(v1) Proton Magnetic Resonance and Stereochemistry of Fractions I and. II 
v11

Page

B. Anhydrodeoxyheptitols from 3,4,6-tr1-0-acetylD-galactal

89

(1) Reaction Conditions 90

(11) Characterisation of Fractions $A$ and $B$ 93

(111) Structures of Fractions A and B 94

(1v) Stereochemistry of Fractions $A$ and $B$ 99

(v) Steric Aspects of Hydroxymethylation 117

C. Hydroformylation of 3,4-d1-0-acety1-D-xylal 120

(1) Reaction Conditions and Product Isolation 121 (1i) Reaction with 2,4-Dinitrophenylhydrazine 124 (111) Aldehydo-hexoses by Oxidation of Hexitols 132

EXPERIMENTAL

General Considerations 136

Experimental Section A 138

3,4-Di-o-acetyl-D-xylaI 138

Dicobalt Octacarbonyl

139

Hydroxymethylation of 3,4-di-0-acetyl-D-xylal

140

Characterisation of Fractions I and II 142

Consumption of Periodate Ion 144

Enantiomer1c 2-deoxy-3-0-(2-hydroxyethyl)-glycerotetritols

2-Deoxy-3-ㅁ-(2-hydroxyethy 1 ) - L-glycero-tetritol 
Page

Attempted Preparations of 2-deoxy-3,4-d1-0-acetylD-xylopyranosyl cyanide

2,3-D1-0-acetyl-1,5-anhydro-4,6-dideoxy-D-arabinoand $\underline{\underline{E}}-\mathrm{x \overline {y }} I 0$-hexitols

Identification of Polyol Y from hydrogenolys is of methyl $\propto$-D-glucopyranoside

160

Reaction of 3,4-di-0-acetyl-D-xylal with Carbon Monoxide and DeuterIum

161

Experimental Section B

164

3,4,6-Tr1-O-acetyI-D-ga lactaI

164

Reaction of 3,4,6-tri-0-acetyl-D-galactal with

Carbon Monoxide and Hydrogen

166

Characterisation of Fractions $A$ and $B$

167

2,6-Anhydro-3-deoxy-4,5-0-isopropyl1dene-D-talo-
heptitol.

2,6-Anhydro-3-deoxy-4,5-0-isopropylidene-1,7-di-

O-p-tolysulphonyl-D-talo-heptitol

170

Reaction with sodium lodide

170

Consumption of Periodate

171

Enantiomeric 2-deoxy-3---(1,3-dihydroxy-2-propyl)glycero-tetritols

Attempted syntheses of optically pure tetrol ethers 174 2-Deoxy-3-0-(1,3-dihydroxy-2-propy 1)-L-glycerotetritol from 2,6-anhydro-3-deoxy-D-giuco-heptitol 179

Experimental Section C

Hydroformylation of 3,4-di-0-acetyl-D-xylal

180

Reaction with 2,4-dinitrophenylhydrazine

181 


\section{Page}

Fraction $\mathrm{Y}$

182

${ }_{Y}^{1}, 5$-Anhydro-4-deoxy-D-arabino-hexitol from Fraction

185

D1-0-acetyl-anhydrodeoxy-aldehydo-hexoses by oxidation of di-0-acetyl-anhydrodeoxy-hexitols

Figure

1

2

3

4
Following page

82

86

97

113 


\section{GENERAL INTRODUCTION}

In order to provide a background to subsequent discussion of the application of the oxo reaction to glycals, a brief review will be made of the oxo reaction of olefins In general, and of the chemistry of the glycals, w1th part1cular emphasis in each case on stereochemical aspects.

\section{The Oxo Reaction}

The reaction of olefins with carbon monoxide and hydrogen in the presence of a cobalt catalyst is commonly referred to as the oxo reaction, as early experiments using ethylene as substrate led to the formation of an appreciable quantity of diethyl ketone. However, the reaction of ethylene is not typical, and in general aldehydes or alcohols are the major products. The stolchlometry of aldehyde formation is as shown in equation $1:$

$$
\mathrm{R} . \mathrm{CH}=\mathrm{CH} \cdot \mathrm{R}+\mathrm{H}_{2}+\mathrm{CO} \longrightarrow \mathrm{R}_{2} \mathrm{CH}_{2} \cdot \mathrm{CHR} \cdot \mathrm{CHO} \perp
$$

This reaction is often referred to as hydroformylation ${ }^{1}$, being formally equivalent to the addition of hydrogen and a formyl group at elther end of the double bond. Alcohol formation in the oxo reaction (equation 2) results from the further reduction of aldehydes formed by the hydroformylation reaction:

$$
\mathrm{R} \cdot \mathrm{CH}_{2} \cdot \mathrm{CHR} \cdot \mathrm{CHO}+\mathrm{H}_{2} \longrightarrow \mathrm{R} \cdot \mathrm{CH}_{2} \cdot \mathrm{CHR} \cdot \mathrm{CH}_{2} \mathrm{OH}
$$


As there is no term in general use to describe the overall conversion of olefins to alcohols (equation $\underline{1}+\underline{2}$ ) it is proposed, for the sake of convenience, to coin the expression "hydrohydroxymethylation" to describe the addition of hydrogen and a hydroxymethyl group to the double bond, and abbreviate this to "hydroxymethylation" in subsequent discussion. It Is noteworthy that this second stage (equation 2) from a mechanistic viewpoint is closely related to hydroformylation, and indeed requires the presence of an appreciable partial pressure of carbon monoxide ${ }^{2}$, evidence that the active catalyst is a cobalt carbonyl.

Historically, the oxo reaction developed from the well known process for hydrocarbon synthesis discovered by Fischer and Tropsch ${ }^{3}$, in which hydrogen and carbon monoxide were passed over an iron catalyst under conditions of high temperature $\left(400-450^{\circ}\right)$ and moderate pressure. It was observed that small amounts of oxygen-containing products were often formed, and in 1929 Smith, Hawk and Golden 4 obtained an increased yield of oxygenated materlal when ethylene was added to the carbon monoxide-hydrogen mixture before passing it over a cobalt catalyst under FischerTropsch conditions. Credit for the development of the oxo reaction as a commerclal process goes largely to Roelen and co-workers of Ruhrchemie A. G. In Germany before and during World War II ${ }^{5}$. By changing the conditions of the 
Fischer-Tropsch synthesis to give a higher pressure and a lower temperature, the reaction of ethylene with water gas (equal volumes of carbon monoxide and hydrogen) was made to yleid a product consisting of propionaldehyde together with some diethyl ketone, no hydrocarbons being formed. Following World War II the oxo gynthesis attracted wide interest, in particular because of its wide range of applicability in the conversion of olefins to aldehydes and alcohols, especially the latter. In general, temperatures between $75^{\circ}$ and $200^{\circ}$, and pressures of synthesis gas from 100 to 300 atmospheres are employed, higher temperatures being usual when alcohols rather than aldehydes are the desired products.

In step with the commerclal development of the oxo" process, much fundamental work has been carried out on this and related reactions catalysed by the metal carbonyls, and their chemistry has been well reviewed from time to time $6,7,8$. Desplte the accumulation of a considerable amount of knowledge of these reactions, many of the finer points of the complex mechanlsms are st1ll not known w1th certainty. In the following pages a brief survey will be made of current views regarding the nature of the catalyst, and the probable role which it plays in the conversion of olefins to aldehydes, and aldehydes to alcohols. 


\section{(1) Nature of the Catalyst}

In the early stages of development of the oxo synthesis In Germany the catalyst used was the conventional FischerTropsch surface catalyst, consisting of a mixture of metallic cobalt and Kleselguhr, together with small amounts of thorlum and magnesium oxides. It became evident to Roelen and coworkers that all the components of the Fischer-Tropsch catalyst with the exception of cobalt were superfluous, and that the active catalyst was probably a soluble carbonyl of cobalt formed by the in situ reaction of the metal with synthesis gas. This was confirmed by subsequent investigations of the reaction on a laboratory scale ${ }^{1,2}$. Adkins and Kresk ${ }^{9}$, who Introduced the use of preformed dicobalt octacarbonyl as catalyst, demonstrated that the hydroformylation reaction was insensitive to sulphur poisoning, further evidence for the homogeneous nature of the catalysis. The fact that the oxo reaction is catalysed by a cobalt carbonyl. contributes to the commercial importance of the process since the form In which cobalt is added is not too important, as would be the case with a conventional solid phase catalyst. In practice, crude organic salts such as the octanoate or naphthenate are often used. The divalent cobaltous ion is first reduced to the metal by hydrogen,

$$
\mathrm{H}_{2} \rightarrow 2 \mathrm{H}^{+}+2 \mathrm{e} ; \quad \mathrm{Co}^{++}+2 \mathrm{e} \rightarrow \mathrm{Co} \underline{3}
$$


and the metal then reacts with carbon monoxide to form dicobalt octacarbonyl
$2 \mathrm{CO}+$
$8 \mathrm{CO} \rightleftharpoons \mathrm{Co}_{2}(\mathrm{CO})_{8}$
$\underline{4}$

Hence, the presence of both carbon monoxide and hydrogen are required to convert cobalt salts to the carbonyl.

A considerable amount of evidence has accumulated which indicates that cobalt hydrotetracarbonyl, $\mathrm{HCO}(\mathrm{CO})_{4}$, rather than dicobalt octacarbonyl, is effective in initiating the oxo reaction. The hydrotetracarbonyl is formed by reaction of hydrogen with dicobalt octacarbonyl

$$
\mathrm{Co}_{2}(\mathrm{CO})_{8}+\mathrm{H}_{2}^{\prime} \rightleftharpoons 2 \mathrm{HCO}(\mathrm{CO})_{4} \quad \underline{5}
$$

Th1s step is thus of fundamental importance in that it involves the activation of molecular hydrogen ${ }^{10}$, which is transferred from the gas to the liquid phase. Orchin and coworkers $^{11}$ have shown that the hydrotetracarbonyl is present under oxo conditions in the absence of olefin; but when olefin is present no cobalt hydrotetracarbonyl is detectable (as the cobalt tetracarbonyl anion $\left[\mathrm{Co}(\mathrm{CO})_{4}\right]^{-}$) until hydroformylation of the olefin is complete, when an appreciable amount of the hydrotetracarbonyl again appears in the reaction mixture.

(11) Mechanism of the Oxo Reaction

Evidence for the mechanism of the oxo reaction as 
carried out under conditions of high temperature and pressure is largely speculative, and is based on the results of reactions between olefins and cobalt hydrotetracarbonyl at atmospheric pressure and temperature. Early mechanistic theories $1,12,13$ did not take into account the now well-proven Intermediacy of cobalt hydrotetracarbonyl, and no longer appear to be relevant. An early finding of importance ${ }^{12}$ was that the rate of hydroformylation varied inversely with increase in the partial pressure of carbon monoxide at constanthydrogen pressure. The stoichiometry of the reaction of olefins with cobalt hydrotetracarbonyl and carbon monoxide at room temperature was investigated by orchin and co-workers 14,15, and with a moderate excess of olefin ( 1 -pentene) under 1 atmosphere of carbon monoxide was found to be

$$
2 \mathrm{HCo}(\mathrm{CO})_{4}+\mathrm{CO}+\text { olefin } \longrightarrow \mathrm{Co}_{2}(\mathrm{CO})_{8}+\text { aldehyde } \underline{6}
$$

The relative rates of reaction of various olefins under these conditions ${ }^{15}$ was found to parallel their rates of hydroformylation under oxo conditions ${ }^{16}$. The reaction of olefins with cobalt hydrotetracarbonyl at room temperature. and below was further explored by Heck and Breslow 17,18 , on whose work current views regarding the mechanisms of the oxo reaction, separately discussed below for hydroformylation and hydrogenation, are largely based. 
(a) Hydroformylation

Subsequent to the generation of cobalt hydrotetracarbonyl by the hydrogenolysis of dicobalt octacarbonyl (equation 5 ), the conversion of olefins to aldehydes is regarded as proceeding in three distinct stages; 1. formation of a $\pi$-complex between olefin and cobalt hydrocarbonyl, which rearranges so that a carbon-metal sigma bond is formed; 2. Insertion of carbon monoxide between metal and carbon, and 3. hydrogenolysis of the resulting complex to give an aldehyde.

1. Heck and Breslow ${ }^{17}$ consider that this first stage Involves at least three distinct steps, as follows

$$
\begin{aligned}
& \mathrm{HCO}(\mathrm{CO})_{4} \rightleftharpoons \mathrm{HCO}(\mathrm{CO})_{3}+\mathrm{CO} \text { I } \\
& \mathrm{R} \cdot \mathrm{CH}=\mathrm{CH} \cdot \mathrm{R}+\mathrm{HCO}(\mathrm{CO})_{3} \longrightarrow\left[\begin{array}{c}
\mathrm{R} \cdot \mathrm{OH}=\mathrm{CHR} \\
\mathrm{HOO}_{\mathrm{O}}(\mathrm{CO})_{3}
\end{array}\right] \underset{\cdots}{\longrightarrow} \mathrm{RCH}_{2} \cdot \mathrm{CHR} \cdot \mathrm{Co}(\mathrm{CO})_{3} \\
& \underline{8} \\
& \mathrm{RCH}_{2} \cdot \mathrm{CHR} \cdot \mathrm{Co}(\mathrm{CO})_{3}+\mathrm{CO} \rightleftharpoons \mathrm{RCH}_{2} \cdot \mathrm{CHR} \cdot \mathrm{Co}(\mathrm{CO})_{4} 9
\end{aligned}
$$

Their view that cobalt hydrotricarbonyl, rather than the hydrotetracarbonyl, is the reactive species is based on evidence that the formation of alkylcobalt tetracarbonyls is inhibited by carbon monoxide; more fundamentaliy, initial complexing with olefin would presumably require the participation of a co-ordinately-unsaturated carbonyl. 
2. Heck and Breslow ${ }^{18}$ found that methylcobalt tetracarbonyl absorbed exactly one mole of carbon monoxide to give a product with a strong band in the infrared at 1728 $\mathrm{cm}^{-1}$, assigned to the acylcobalt Iinkage, R.CO.Co. The same infrared absorption at reduced intensity was also shown by solutions of alkylcobalt tetracarbonyls, indicating that these complexes were in equilibrium with acylcobalt tricarbonyls,

$\mathrm{RCH}_{2} \cdot \mathrm{CHR}_{\mathrm{p}} \mathrm{CO}(\mathrm{CO})_{4} \rightleftharpoons \mathrm{R} \cdot \mathrm{CH}_{2} \mathrm{CHR} \cdot \mathrm{CO} \cdot \mathrm{Co}(\mathrm{CO})_{3} \stackrel{\mathrm{CO}}{\rightleftharpoons} \mathrm{RCH}_{2} \cdot \mathrm{CHR} \cdot \mathrm{CO} \cdot \mathrm{Co}(\mathrm{CO})_{4}$

Evidence has been obtained with analogous complexes of manganese ${ }^{19}$, using $\mathrm{C}^{14}$-labelied carbon monoxide, which indicates that the inserted carbonyl was originally bonded to the metal. The insertion reaction is thus essentially the migration of an alkyl group from metal to the carbon atom of a carbonyl ligand.

3. The reaction of acetylcobalt tetracarbonyl with cobalt hydrotetracarbonyl under room temperature conditions afforded acetaldehyde and dicobalt octacarbonyl in good $\mathrm{y} 1 \mathrm{e} ! \mathrm{d}^{18}$

$$
\mathrm{CH}_{3} \mathrm{CO} . \mathrm{CO}(\mathrm{CO})_{4}+\mathrm{HCO}(\mathrm{CO})_{4} \longrightarrow \mathrm{CH}_{3} \mathrm{CHO}+\mathrm{CO}_{2}(\mathrm{CO})_{8} \quad \text { II }
$$

This reaction is not, however, considered to operate under oxo conditions as, despite the fact that acetylcobalt 
tetracarbonyl is also reduced to aldehyde by hydrogen under pressure, the reaction is completely inhibited by carbon monoxide. To account for the final stage of hydroformylation, Heck and Breslow suggest the intermediacy of coordinately-unsaturated acylcobalt tricarbonyls, which are reduced to aldinydes by hydrogen or converted to unreactive tetracarbonyls by carbon monoxide. The well-known ${ }^{12}$ adverse effect of carbon monoxide on the course of the oxo reaction can therefore be attributed to this competition.

$$
\begin{array}{ll}
\mathrm{RCH}_{2} \cdot \mathrm{CHR} \cdot \mathrm{CO} \cdot \mathrm{Co}(\mathrm{CO})_{3} & +\mathrm{H}_{2} \longrightarrow \mathrm{RCH}_{2} \cdot \mathrm{CHR} \cdot \mathrm{CHO}+\mathrm{HCO}(\mathrm{CO})_{3} \frac{12}{\mathrm{CO} \int} \\
\mathrm{RCH}_{2} \cdot \mathrm{CHR} \cdot \mathrm{CO} \cdot \mathrm{Co}(\mathrm{CO})_{4} & \mathrm{HCO}(\mathrm{CO})_{3}+\mathrm{CO} \rightleftharpoons \mathrm{HCO}(\mathrm{CO})_{4}
\end{array}
$$

\section{(b) Hydrogenation}

A scheme which is analogous to that described above for hydroformylation 17,18 has been proposed by Marko 20 for the subsequent hydrogenation of aldehydes to alcohols under oxo conditions (equation $\underline{2}$ ). Co-ordinately-unsaturated carbonyls are considered to be the reactive intermediates; thus, cobalt hydrotricarbonyl forms a $\pi$-complex with the aldehyde, which rearranges to an alkoxycobalt tricarbonyl (equation 13). Marko suggests that this is then hydrogenolysed by molecular hydrogen to give the alcohol, or is competed for by carbon monoxide, giving rise to an unreactive tetracarbony 1 


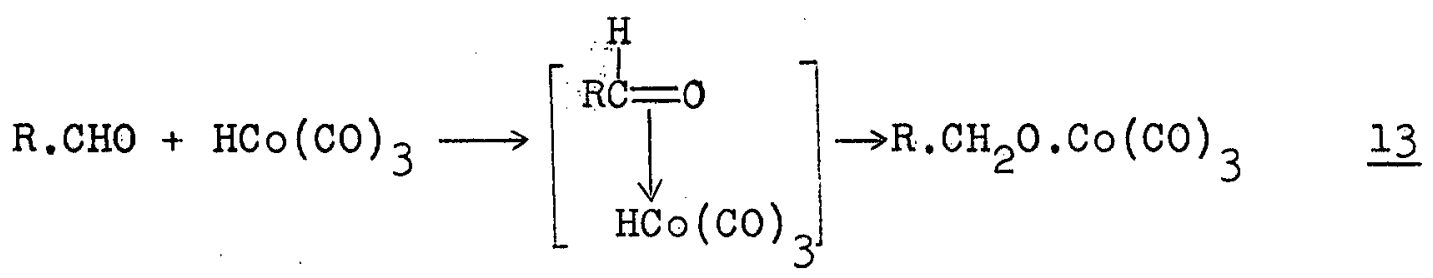

$$
\begin{aligned}
& \mathrm{R} . \mathrm{CH}_{2} \mathrm{O} . \mathrm{Co}(\mathrm{CO})_{3}+\mathrm{H}_{2} \longrightarrow \mathrm{R} \cdot \mathrm{CH}_{2} \mathrm{OH}+\mathrm{HCO}(\mathrm{CO})_{3} \ldots \\
& \text { col| } \\
& \mathrm{R} \cdot \mathrm{CH}_{2} \mathrm{O} \cdot \mathrm{Co}(\mathrm{CO})_{4}
\end{aligned}
$$

The views of Aldridge and Jonassen 21,22 differ from those of other workers in that they regard hydroformylation, and hydrogenation of aldehydes, to be heterogeneously catalysed reactions in that the in situ formation of cobalt hydrotetracarbonyl by the hydrogenolysis of dicobalt octacarbonyl (equation 5) is catalysed by cobalt metal, which they consider to be present in equilibrium with the soluble octacarbonyl (equation 4 ). Their concept of the subsequent stages is, however, fundamentally similar to those of Heck and Breslow 17,18 and Marko ${ }^{20}$.

\section{(iii) Effect of Olefin Structure on Hydroformylation}

(a) Effect on Rate of Reaction

All simple olefins have been found to undergo the oxo reaction, although the rate of reaction is observed to be highly dependent on the structure of the olefin. Workers at the U.S. Bureau of Mines ${ }^{16}$ have made a comprehensive study of the influence of structure on the rate of hydroformylation at $110^{\circ}$, using 26 olefinic hydrocarbons, and 
a 50-fold variation was observed between the fastest and slowest rates. The results in all cases appear to demonstrate a clear relationship between reaction rate and degree of steric hindrance about the double bond, the latter factor presumably reflecting the ease of formation of an intermediate complex with cobalt hydrotricarbonyl. Stratght chain terminal olefins were observed to react most readily; with little decrease in rate with increasing chain length. The rate for straight chain internal olefins was about one third that for the terminal olefins; however, the exact position of the double bond, providing it was internal, had little influence on rate. Branching of the carbon chain always resulted in a decrease in reaction rate, even for olefins having a single methyl group remote from the double bond, as in 4-methyl-1pentene. The presence of a methyl group at one of the carbon atoms of the double bond reduced the rate 10-fold, for example in going from 1-pentene to 2-methyl-1-pentene. The slowest rates were observed with branched internal olefins: thus the rate of hydroformylation of 2,3-dimethyl-2-qutene, in which the double bond is completely substituted by methyl groups, was approximately $1 / 50$ th that of an unbranched terminal olefin. The rates observed for cyclic olefins are of interest in that, whereas cyclopentene and cycloheptene reacted faster than the corresponding acyclic internal olefins, the reaction rate for cyclohexene was 
appreclably slower. This observation has been explained 6 on the basis that both cyclopentene and cycloheptene are In a more highly strained state (strain energies of 4.4 and $4.1 \mathrm{kcal} / \mathrm{mole}$ respectively relative to cyclohexene). Traynham ${ }^{23}$ has pointed out that in all known cases the more strained is a cyclic structure, the more reactive it is in electron donating roles. One might therefore expect the $\pi$ electrons of cyclohexene to be less avaliable for donation to the vacant $d$ orbitals of cobalt in complex formation.

(b) Effect on Mode of Addition

In the hydroformylation of unsymmetrical olefins, avallable evidence indicates that the formy group adds to the least hindered side of the double bond under normal conditions of high temperature and pressure, (although this is not necessar1ly the case for reactions with cobalt hydrotetracarbonyl at room temperature ${ }^{17}$ ); thus olefins having the structure $\mathrm{R}-\stackrel{\mathrm{CH}}{\mathrm{C}}=\mathrm{CH}_{2}$ give predominantly the terminal aldehyde. For example, the major product from the hydroformylation of isobutylene is isovaleraldehyde, together with a small proportion of trimethylacetaldehyde ${ }^{24}$.

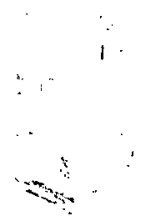<smiles>CC(C)=[C+][O-]</smiles><smiles>C1CCCCC1</smiles><smiles>CC(C)C=CC(C)[O+]=CCC(C)C</smiles> 
of particular interest is the distribution of products obtained from the application of the oxo reaction to cyclic vinylic ethers, as these compounds are structurally related. to the glycals. The hydroxymethylation of 2,3-dihydro-4Hpyran (1) and certain of 1 ts derivatives has been investigated recently by Falbe and Korte 25 . The reactions were performed under conditions $\left(190^{\circ}, 300\right.$ atmospheres of synthesis gas $(1: 1)$ ) leading to the complete conversion of aldehydes to alcohols, thereby facilitating the identification of reaction products. Reaction of 2,3-dihydro-4Hpyran (1) with carbon monoxide and hydrogen resulted predominantly in attachment of the hydroxymethyl group to the side of the double bond adjacent to the ring oxygen to give 2-hydroxymethyl-tetrahydropyran (2) in $78 \%$ yield; In addition; a small proportion of 3-hydroxymethyl 2 tetrahydropyran (3) was 1solated, together with a smaller amount of tetrahydropyran (4) resulting from hydrogenation of the double bond<smiles>C1=COCCC1</smiles>

(1)

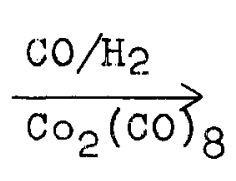

(2)

$78 \%$<smiles>OC[C@H]1CCCCO1</smiles><smiles>[GeH2]=[GeH2]</smiles>

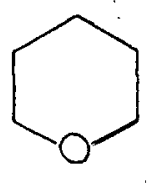

(4)

Under similar conditions 2-hydroxymethyl-2,3-dihydro4H-pyran (5) gave exclusively 2,6-b1s-hydroxymethyltetrahydropyran (6) 
<smiles>OCC1CCC=CO1</smiles>

(5)

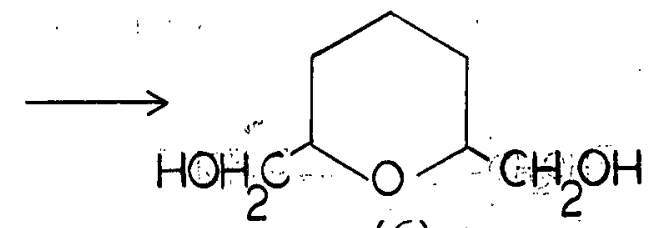

(6)
17

When the carbon atom of the double bond adjacent to the ring oxygen bore a methyl substituent, as in 2,6-dimethyl2,3-dihydro-4H-pyran (7), then the direction of addition was reversed and 3-hydroxymethyl-2,6-dimethyl-tetrahydropyran (8) was isolated, but in lower yield<smiles>CC1=CCC[C@@H](C)O1</smiles>
(7)<smiles>CC1CCC(CO)C(C)O1</smiles>

(8)

Comparable results have previously been obtained by the hydroxymethylation of furan (9). This compound reacted as a typical conjugated diene, in that one double bond was hydrogenated while the other underwent the normal reaction with carbon monoxide and hydrogen ${ }^{26}$; addition of the hydroxymethyl group occurred at $\mathrm{C}-2,2$-tetrahydrofurfuryl alcohol (10) being isolated in 35\% yield.<smiles>O=C[OH+]c1ccco1</smiles>

(9)

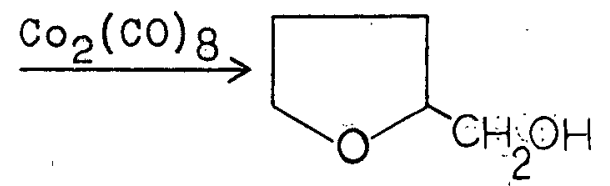

(10) 
However, when both positions adjacent to the ring oxygen were blocked, hydroxymethylation occurred at the alternative site. Thus, 2,5-dimethylfuran (11) gave 2,5-dimethyl-3tetrahydrofurfuryl alcohol (12) 27 .<smiles>Cc1ccc(C)o1</smiles>

(11)<smiles>C1CC1</smiles>

A reaction which closely resembles hydroformylation is the hydrocarboxylation of olefins in the presence of nickel carbonyl

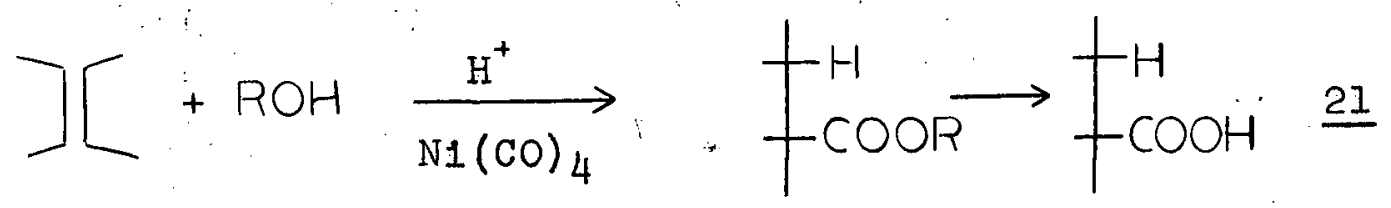

Bird and co-workers ${ }^{28}$ found recently that strained olefins such as norbornene (13) underwent hydrocarboxylation at atmospheric pressure and temperature. By the use of deuterated solvents (deuterium oxide, deuterioethanol and deuterioacetic acid) It was shown that (13) was converted into 3-exo-deuterio-b1cyc10-[2.2.1] -heptane-2-exo-carboxylic acid (14). These workers suggest that, in hydrocarboxylation,

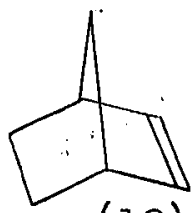

(13)

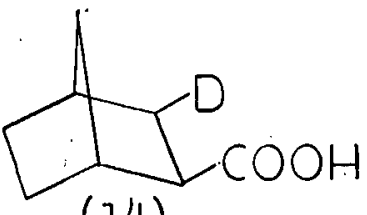

(14) 
c1s addition to olefins from the least hindered side is probably general.

Two groups of workers 29,30 have investigated the preparation of 6-methyl sterolds by the application of the oxo reaction to sterolds having a double bond in the $\triangle^{5}$-position (15). The product in each case was the $6-\alpha$-hydroxymethylallosteroid (16), and it was concluded that "the oxo reaction<smiles>[R]C1CCC2(C)C(=CCC(C)C2C)C1</smiles>

(15)

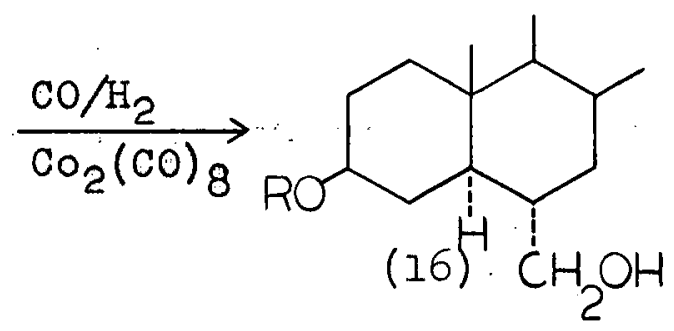

under hydroxymethylation conditions appears to take place by a cis addition" 29 .

The Glycals

(1) Structure of the Glycals

The glycals, discovered in 1913 by Fischer and Zach ${ }^{31}$; owe their name to Fischer's observation that the first (Impure) preparations of D-glucal gave characteristic aldehyde reactions. When 1 t was later realized that pure glycals are not aldehydic compounds, the name was flrmly established. A proposal to systematise glycal nomenclature, 
at present under official consideration (of reference 32 ), regards the glycals as 1,5-anhydro-derivatives of 1-enols; thus 3,4,6-tr1-0-acetyl-D-galactal (40) would be renamed 3,4,6-tr1-0-acetyl-1,5-anhydro-2-deoxy-D-1yxo-hexose-1-enol.

Glycals are cycl1c carbohydrates characterised by the presence of a $\mathrm{CH}=\mathrm{CH}$ IInkage between carbon atoms 1 and 2. They can be regarded formally as derlved from the corresponding aldose by removal of two hydroxyl groups from adfacent carbon atoms, (e.g. D-galactal (19) from D-galactose $(18))$, and In this respect differ from the other well known class of unsaturated carbohydrates, the glycoseens, which structurally are derived from the corresponding aldose by removal of a molecule of water. The latter group includes the so-called 2-hydroxyglucals (e.g. 2-hydroxy-D-galactal (17)), or 1,2-glycoseens, as well as the 5,6-glycoseens which have an exocyclic double bond between carbon atoms 5 and 6.

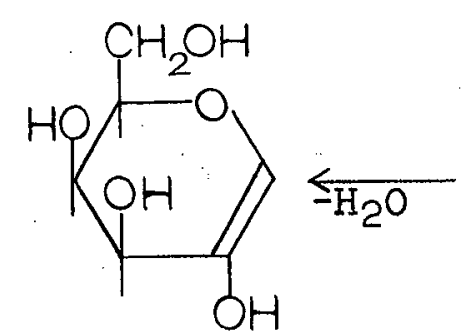

(17)

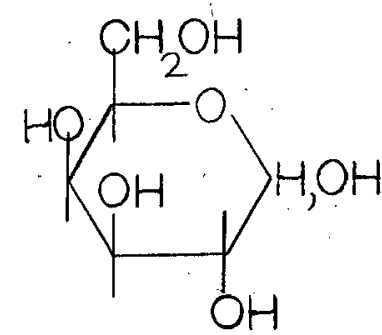

(18)

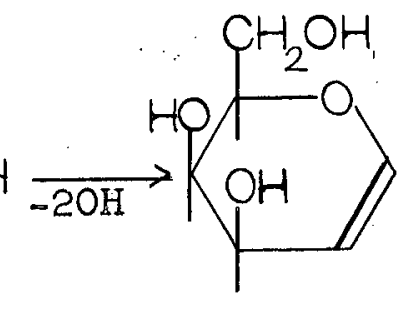

(19)

Aldoses which are eplmerle at $\mathrm{C}-2$, such as D-glucose and D-mannose, give the same glycal, as asymmetry at $\mathrm{C}-2$ 
(and C-I) is lost on formation of the double bond; the name of the resulting glycal is usually derived from that of the more common of the two parent aldoses. Thus the elght stereolsomeric hexoses of the D-series can give rise to four "hexals"; D-glucal, D-galactal, D-allal and D-gulal (no preparations of the latter two are known), and two "pentals", (D-xylal and $\underline{\underline{D}}$-arabinal) are possible from the four D-pentoses. With the exception of a novel "furanal" reported recently ${ }^{32}$, all the known glycals are six-membered ring structures derived from pyranose sugars.

The structure of the best known glycal, 3,4,6-triO-acetyl-D-glucal (20 a) was worked out at an early stage 33,34 . The presence of a double bond was demonstrated by the addition

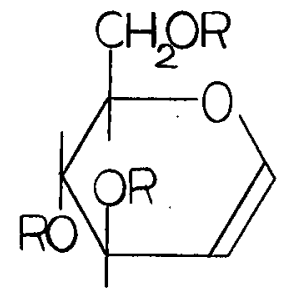

(20a) $R=A C$

(20b) $\quad \mathrm{R}=\mathrm{H}$

of two atoms of bromine or hydrogen; its position, between carbon atoms 1 and 2 , was shown by the fact that cleavage with ozone liberated D-arabinose, formation of the latter pentose also providing evidence that the configurations of carbon atoms 3,4 and 5 are unchanged from those of Dglucose. The structures of other glycals have been proved 
In a similar manner. The presence of the double bond in the $s 1 x$-membered ring of glycals results in carbon atoms 1,2 and 3 and the ring oxygen atom being constrained into the so-called half-chain conformation of the cyclohexene ring. The presence of substituents on the ring permits the possibility of two halpf-chair conformations or the glycals; thus, in the case of one conformation (21 a) for 3,4,6-tr1-0-acety1-D-glucal, substituents at $\mathrm{C}-3, \mathrm{C}-4$ and C-5 are in equatorial or pseudo-equatorial positions, whereas in the alternative conformation (2l b) these substituents are in the less stable axial or pseudo-axial orlentations. That 3,4,6-tri-0-acetyl-D-glucal adopts the

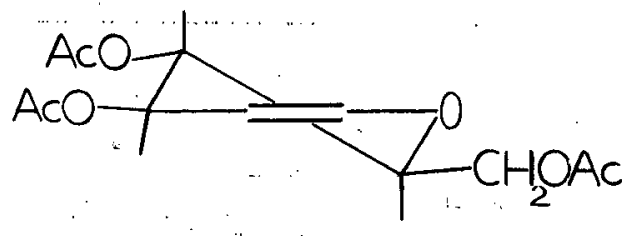

$(21 a)$

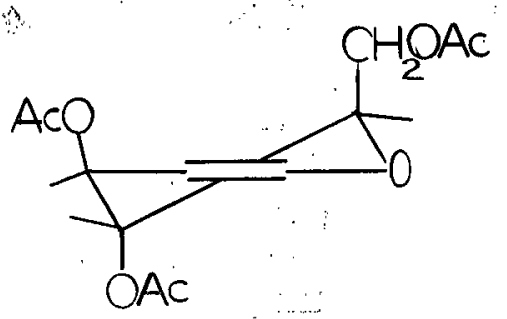

(2ib)

more stable half-chair conformation (2la) in solution was confirmed recentiy by Hall and Johnson ${ }^{35}$. Using proton magnetic resonance spectroscopy at high radio frequency (100 Mc/s) these workers were able to measure the chemical shift of each proton, and the coupling constants between adjacent protons, certain of their assignments being confirmed 
by double resonance experiments. It was then possible to relate the coupling constants of adjacent ring protons to their dihedral angle by application of Karplus' equation ${ }^{36}$, and thus arrive at the approximate geometry of the ring. The results showed that the dihedral angle between $\mathrm{C}-\mathrm{H}$ (3) and $\mathrm{C}-\mathrm{H}(4)$ is approximately $140^{\circ}$, and that between $\mathrm{C}-\mathrm{H}$ (4) and $\mathrm{C}-\mathrm{H}(5)$ is about $150^{\circ}$, and therefore confirmed that the glycal adopts the conformation (21 a) in solution. As the latter dihedral angle was less than $180^{\circ}$, some "flattening" of the ring was indicated. No comparable measurements have yet been made of the conformations of the other avallable glycals; certainly it is reasonable to predict that the half-cha1r conformation of 3,4-d1-0-acetyl-D-xylal (22) would be analogous to that found for 3,4,6-tri-0-acetylD-glucal.

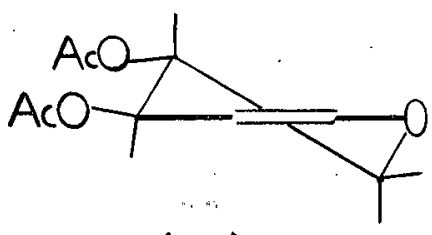

(22)

(11) Preparation of the Glycals

Despite the fact that over 50 years have elapsed since Fischer and $\mathrm{Zach}^{31}$ first synthesised 3,6,6-tri-0-acetyl-Dglucal, their procedure, with minor modiflcations designed 
to Improve yields, is still the only one of signifleance for the preparation of glycals. It appears probable 37 that Fischer's original intention on treating 2,3,4,6-tetra-0acetyl- $\alpha-D$-glucosyl bromide (23) with zinc and acetic acid was reductive dehalogenation to afford a derlvative of $1,5-$ anhydro-D-glucitol; in actual fact a good yield of 3,4,6tr1-0-acetyl-D-glucal (20 a) was obtained,
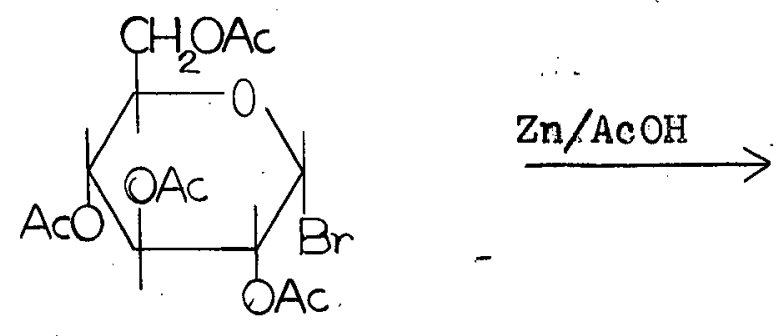

(20a)

(23)

A mechanism to explain glycal formation has been suggested by Prins ${ }^{38}$, and discussed by overend and stacey ${ }^{39}$. The carbonium ion intermediate (25) resulting from ionisation of the acetobromoaldose (24) can either react with solvent to give the acetylated aldose (26), or can acquire two electrons from the metal to furnish the carbanion (27), which affords the glycal by elimination of an acetoxy group from $\mathrm{C}-2$. It is also conceivable that the carbanion may acquire a proton to give the 1,5-anhydroalditol (29), although the latter do not appear to have been detected as products of this reaction. 


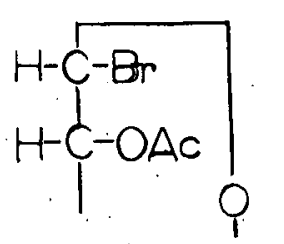

(24)<smiles>CC1CCCCC1OOC(=O)O</smiles>

(29)

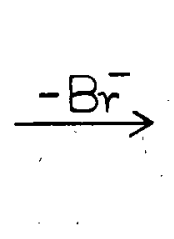

$\mathrm{H}-\mathrm{CH}$
$\mathrm{H}-\mathrm{C}-O A C$

$(25)$

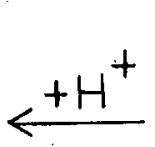

$\stackrel{-\Delta \bar{c}}{\longrightarrow}$

(27)

$\stackrel{+R}{\longrightarrow} \stackrel{H-C-R}{H-O A C}$

(26) $\mathrm{R}=\mathrm{OH}$ or $\mathrm{OAC}$

26

Because of the Importance of glycals as intermediates in carbohydrate chemistry, in particular in the preparation of 2-deoxy sugars 39 , various modifications to Fischer's original procedure have been introduced with the object of improving the overall yields of the sequence: aldose $\longrightarrow$ acetobromoaldose $\longrightarrow$ glycal $\longrightarrow$ 2-deoxy-aldose. A standard method for the preparation of acetobromoaldoses was passage of hydrogen bromide through a solution or suspension of the aldose in acetic anhydride. A significant improvement over the direct use of hydrogen bromide was Introduced by Barcza1-Martos, and Korösky 40 , who generated the gas 1n s1tu by adding phosphorus tribromide and water, or more simply phosphorus, barine and water.to torolution of the fully-acetylated aldose in acetic anhydride. Good yields of several crystalline acetobromoaldoses were obtained in this way, without intermediate isolation of the fully-acetylated precursors. 
Various modifications have been made to Fischer's original procedure for reduction of the acetobromo-sugar with zinc and acetic acid. Deriaz and co-workers ${ }^{41}$ introduced the use of chloroplatinic acid which, added at intervals to the reduction mixture, maintained a vigorous reaction and enabled lower temperatures to be employed. Significant improvements in the yields of 3,4-di-0-acetyl-D$\operatorname{arabina}^{4 I}$ and $3,4,6-\operatorname{trl}_{1}-0$-acetyl-D-galactal ${ }^{42}$ were attained in this way. Iselin and Relchstein 43 added sodium acetate to buffer the zinc-acetic acid mixture and to remove hydrogen bromide formed during the course of the reaction, and activated the zinc by addition of copper as the sulphate. The most convenient general procedure for glycal preparation is that of Helferich and co-workers 44,45 , who combined the advantages of the acetobromoaldose preparation of Barcza1-Martos and Korösky ${ }^{40}$, and the reduction procedure of Iselin and Reichstein ${ }^{43}$; the conversion of aldose to acetylated glycal is carried through in one stage without isolation of intermediates.

\section{(ii1) Reactions of the Glycals}

Practically all the reactions of the glycals have their counterpart in well known reactions of olefins in general, Involving addition across the double bond. In the majority of cases the direction of addition is influenced 
by the proximity of the ring oxygen atom. Glycals react as typical vinyl ethers, and frequently exhibit an interesting resemblance to the six-membered cyclic vinyl ether, 2,3-dihydro4H-pyran (1). Typical electrophilic addition to a vinyl ether results in addition of the electrophile to the carbon of the double bond $\beta$ to the ether oxygen, as the resulting cation is stabilised by the electron-rich oxygen. Acid-catalysed formation of the well known tetrahydropyranyl ethers (30) thus proceeds as follows 46

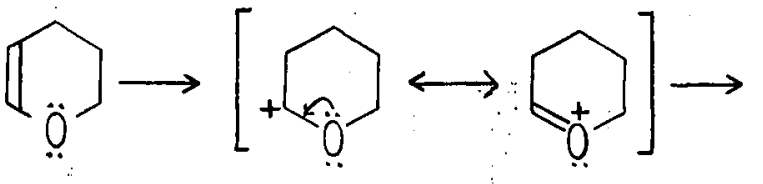

(1)<smiles>[R]C1CCCCO1</smiles>

(30)

The ring oxygen of glycals is seen to exert a similar directIng influence on the course of additions to the double bond; moreover, steric factors due to substituents at $c-3$ appear to have a marked effect in determining the distribution of isomeric products. Examples can be taken from a variety of addition reactions of glycals to illustrate these points.

Ionic additions of reagent of the general form HX (where $\mathrm{X}=\mathrm{OH}$, alkoxy, halogen) to the double bond of glycals are well known reactions for the preparation of 2-deoxy aldoses 
and their derivatives. The ac1d-catalysed addition of water to afford 2-deoxy sugars has been studied in deta1139;

typically the conversion is effected in cold, dilute sulphuric acid solution. It was considered by: Isbell and Pigman 47 that under these conditions 2-deoxy-D-galactose was formed from D-galactal (19) via an intermediate sulphate ester which was subsequently hydrolysed on heating with barium carbonate; however, this was disproved by overend and co-workers ${ }^{48}$. An alternative mechanisim involving proton-catalysed opening of the oxygen bridge has been suggested ${ }^{49}$. The accepted mechanlism of electrophilic addition to vinyl ethers ${ }^{46}$, exemplified by the formation of tetrahydropyranyl ethers (30) (equation 27) would appear to be applicable to this and other ionic additions of HX-type reagents to glycals.

Cis Additions to the Double Bond

The reaction of olefins with nitrcs'yl chloride, with the formation of nitroso-derivatives, has been considered 50 to proceed by an Ionic mechanism, with the nitroso group entering into combination as an electrophilic fragment $\mathrm{NO}^{+}$, and the chlorine as nucleophilic $\mathrm{Cl}^{-}$. Meinwald and coworkers ${ }^{51}$ have obtained experimental evidence from a study of the reactions of norbornene and norbornadiene with nitrosyl chloride which casts doubt on the ionic mechanism (apparent c1s addition, lack of incorporation of nucleophilic 
solvent). They suggest a four-centre mechanism of addition with little or no carbonium ion character developing in the transition state

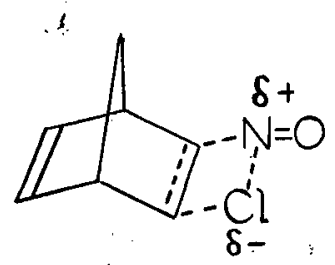

Serfontein and co-workers 52 have obtained highly crystaline adducts by the reaction of nitrosyl chloride with various acetylated glycals. Structural investigations by proton magnetic resonance spectroscopy confirmed that addition to the double bond was c1s. The fact that from 3,4,6-triO-acetyl-D-glucal (20 a), 3,4,6-tri-0-acetyl-2-deoxy-2nitroso- $\alpha$-D-glucopyranosyl chloride (31) was obtained, whereas the $\beta$-D-arabinopyranosyl chloride (33) resulted from the addition of nitrosyl chloride to 3,4-d1-0-acetylD-arabinal (32), would seem to indicate that the $\mathrm{C}-3$ acetoxy group influences the direction of approach of nitrosyl chlorlde to the double bond.

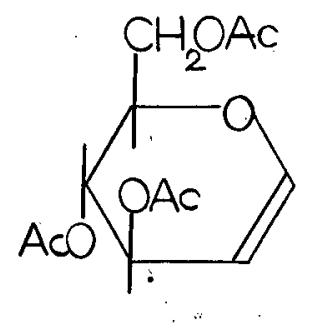

(20a)

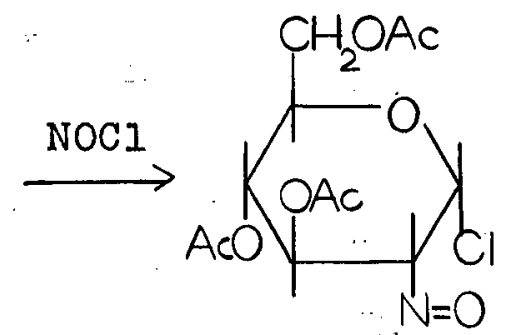

(31) 


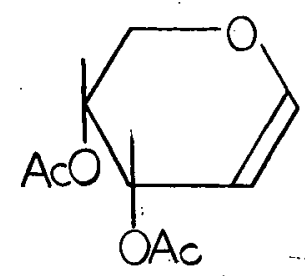

(32)

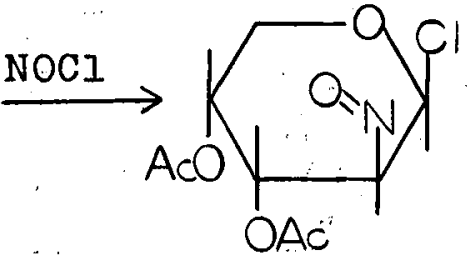

(33)

The light-catalysed reaction of olefins with phenanthraquinone to give 1,4-dioxane derivatives ${ }^{53}$ was applied to the glycals by Helferich ${ }^{4}$. When 3,4,6-tri-oacetyl-D-glaplol (20 a) was thus reacted with phenanthraquinone and the resulting adduct deacetylated, phenenthrenehyaroquinone-D-glucoside anhydride (34) was obtained, from which D-glucose (35) was liberated on ozonolysis. The fact that no D-mannose was obtained from these reactions indicates that addition of phenanthraquinone to the double bond of the glycal took place exclusively from the least hindered side

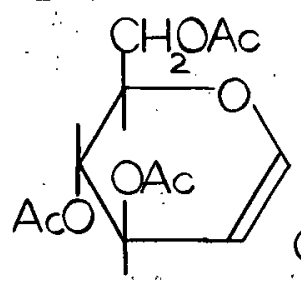

$(20 a)$

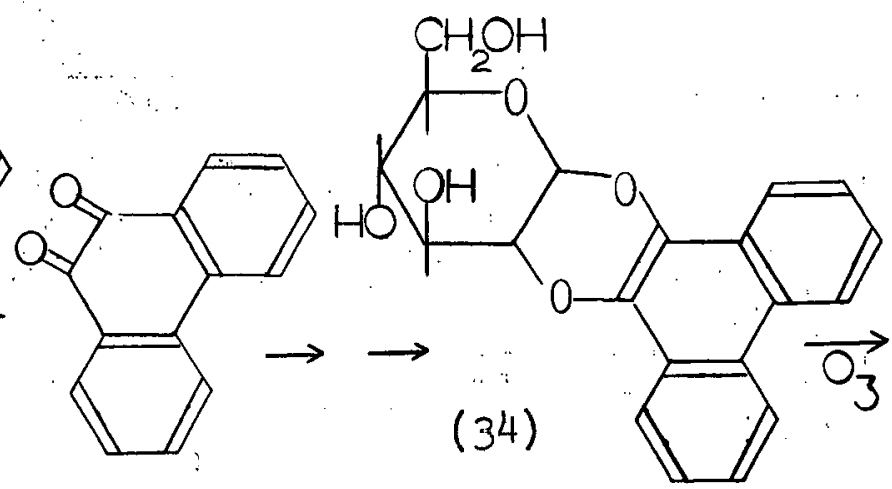

(34)

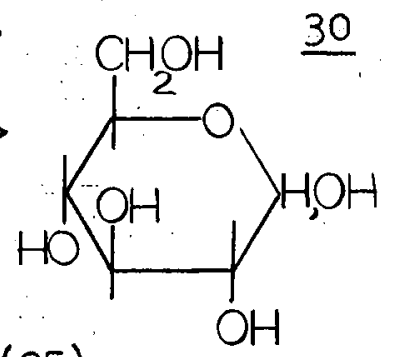

(35)

Similarly, c1s hydroxylation of the double bond of D-glucal (20 b) and 3,4,6-tri-0-acetyl-D-glucal (20 a) by 
osmium tetroxide, proceeding through the cyclic diester of osmic acid (equation 31 ) resulted in predominant formation of the cyclic intermediate at the least hindered side of the double bond, and in both cases more D-glucose than D-mannose was obtained on hydrolysis 55<smiles>CC(=O)C(C)=C(C)C</smiles>

(b) Trans Additions to the Double Bond

Although less relevant in considering possible steric effects likely to be operative in the hydroformylation of glycals, a number of reactions involving trans addition across the double bond of acetylated glycals are known in which a similar directing influence appears to be exerted by the C-3 acetoxy substituent. The reactions in question are considered to proceed through cyclic intermediates which open by rearward attack of a nucleophile to give a trans product. It is known that in cyclic systems the preferred steric course of trans addition is such as to favour the diaxial product 56 . Thus, in the case of the glycals, where nucleophilic addition is at $\mathrm{C}-1$, trans diaxial opening of a cyclic intermediate requires that the latter be in a 
$\beta$-orlentation (above the plane of the ring) (36). Conversely, a trans-diequatorial product must arise from a cyclic intermediate (37) which is oriented below the plane of the pyranoid ring

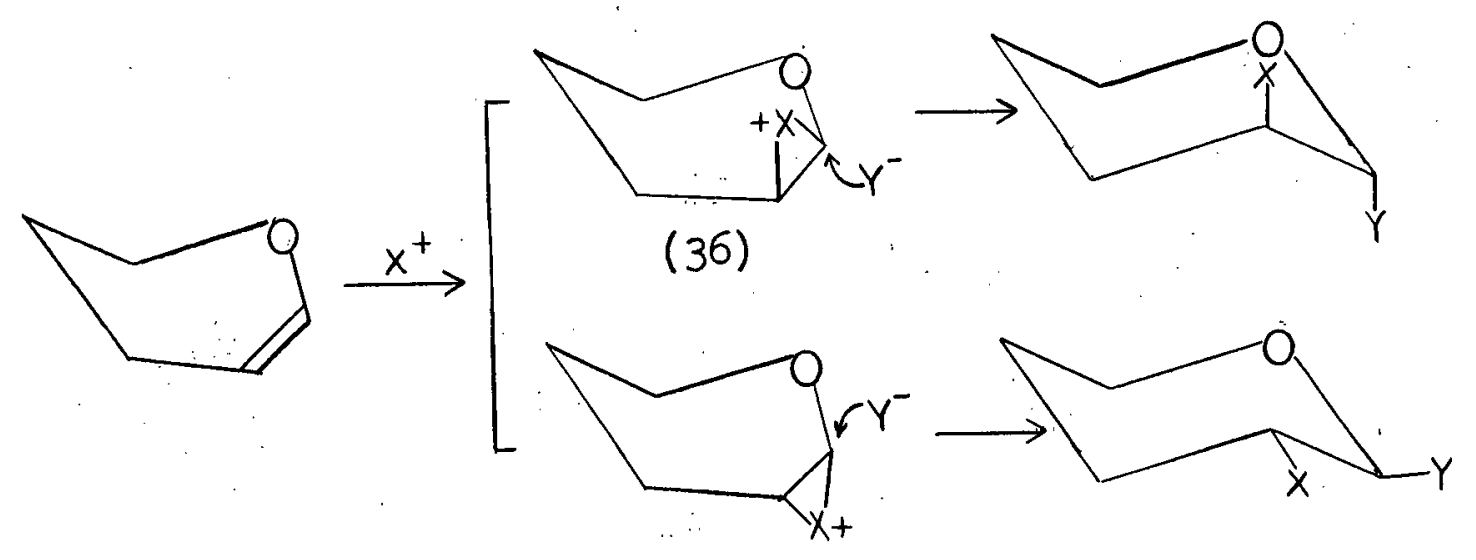

(37)

The addition of mercuric salts to olefins is usually considered to proceed via a mercurinium Ion (e.g. (38)), which reacts with nucleophilic solvent to give a trans product $^{57}$ : From 3,4,6-tri-0-acety1-D-glueal (20 a), Manolopoulos and co-workers ${ }^{58}$ obtained, on reaction with mercuric acetate in methanol, a crystalline compound identified as methyl 3,4,6-tri-0-acetyl-2-acetoxymercur1-2-deoxy$\beta$-D-glucopyranoside (39), the trans-diequatorial product

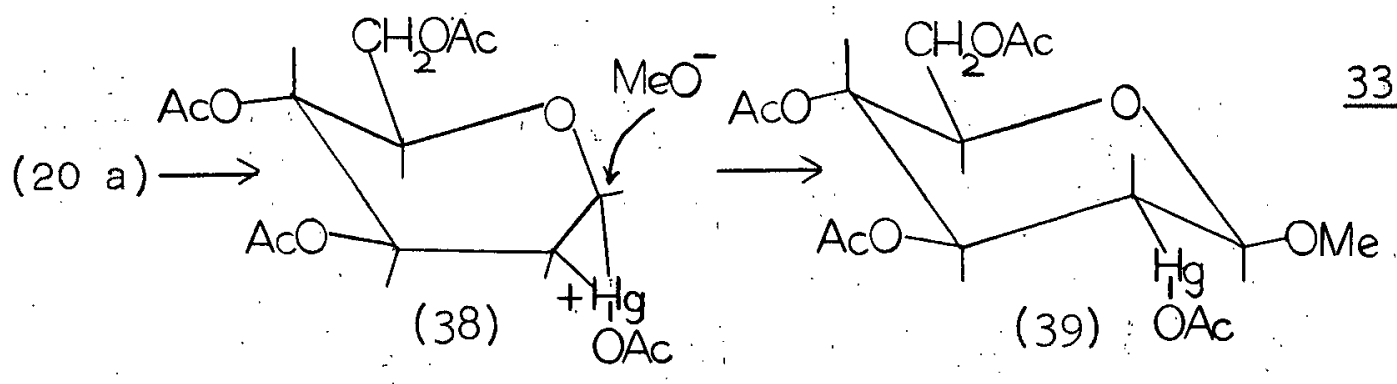


These workers believe that the bulky $\mathrm{C}-3$ acetoxy group tends to shield the double bond from attack at the upper $(\beta)$ side, and the $\alpha$-mercurinium ion (38) becomes important. Rearward approach of methanol to $\mathrm{C}-1$ then leads to (39).

$$
\text { Other reactions of 3,4,6-tri-o-acetyl-D-glucal }
$$
proceeding through analogous mechanism, in which the formation of an equal or major proportion of the kinetically less favoured product having the D-gluco-configuration (c-2 equatorial substituent) Indicates the importance of a cyclic intermediate at the least hindered $(\alpha)$ side of the ring, are with perbenzo1c ac1d (via 1,2-epoxide) 59 , with bromine (via bromonium $10 n)^{60}$, and with iodine and silver benzoate (v1a lodinium ion) ${ }^{61}$.

The Oxo Reaction of Glycals

The reaction of glycals with carbon monoxide and hydrogen under oxo conditions was first explored in 1956 by Rosenthal and co-workers $62,63,64$. Glycals studied were 3,4,6-tr1-0-acetyl-D-galactal (40) and 3,4,6-tr1-0-acetyID-glucal (20 a); In each case saturated seven carbon compounds were obtained and characterized.

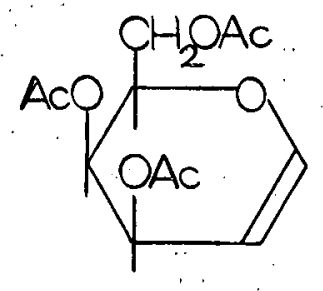


The oxo reaction of glycals therefore represented an additional method for lengthening the carbon chain of carbohydrates 62 . The products obtained from these reactions were acetylated alcohols, rather than aldehydes, and undoubtedly arose by the initial hydroformylation of the glycal double bond, followed by reduction of the formyl group to hydroxymethyl (equations 1 and 2 ). The structures of the products thus obtained were not established, but in the case of the reaction of 3,4,6-tri-o-acetyl-D-galactal it was thought that hydroxymethylation had occurred at $\mathrm{C}-2$ of the giycal, to give a branched-chain carbohydrate ${ }^{63}$; this glycal also appeared to be unique in that only one product was isolated from its reaction with carbon monoxide and hydrogen.

Tork discussed in this thesis extends these earlier investigations, to which further reference will be made in subsequent discussion, to a study of the oxo reaction of the pental, 3,4-d1-0-acetyl-D-xylal (22), and also describes a further investigation of the reaction of $3,4,6$ $\operatorname{tr1-0}$-acetyl-D-galactal (40). 
A. Hydroxymethylation of 3,4-Di-0-acetyl-D-xylal

Previous work 63,64 has demonstrated that the oxo reaction can be applied successfully to the acetylated hexals (20 a) and (40), the major products of the reactions being acetylated alcohols having one more carbon atom than the starting material; thus the reactions appeared to follow the expected course and a hydroxymethyl: group was added to one side of the double bond. It could be anticipated that application of the same reaction to the acetylated pentals would therefore result in the formation of d1-0-acetyl derivatives of six carbon compounds. The object of the work described in this section was to investigate the structures of products formed by the reaction of 3,4-di-0-acetyl-D-xylal (22) with carbon monoxide and hydrogen under hydroxymethylation conditions, whereby, as a result of the absorption of 3 moles of synthesis gas per mole of substrate, the anticipated products are alcohols, rather than aldehydes

$$
-\mathrm{CH}=\mathrm{CH}-+\frac{2 \mathrm{H}_{2}+\mathrm{CO}}{3 \text { moles }} \longrightarrow-\mathrm{CH}_{2}-\stackrel{\mathrm{CH}}{-\mathrm{CH}_{2} \mathrm{OH}} \quad \underline{34}
$$

In a later section (C) experiments w1Il be described in which it was attempted to terminate the reaction at the aldehyde stage (equation 1 ), at the point where 1 mole of 
glycal had absorbed 2 moles of synthesis gas.

\section{(1) Reactants and Reaction Conditions}

(a) 3,4-D1-0-acetyI-D-xyla1 (22)

3,4-D1-0_acetyl-D-xylal (22) was first prepared in 1929 by Levene and Mori 65 , as an intermediate in the synthesis of 2-deoxy- $\beta$-D-xylose, using an adaptation of the original procedure of Flscher and $\mathrm{Zach}^{31}$. Reduction of 2,3,4-tr1-0-acetyl- $\alpha$-D-xylopyranosyl bromide (43) with zinc and 50\% acetic ac1d gave (22) in $60 \%$ yield. Essentially the same procedure has recently been given as a standard

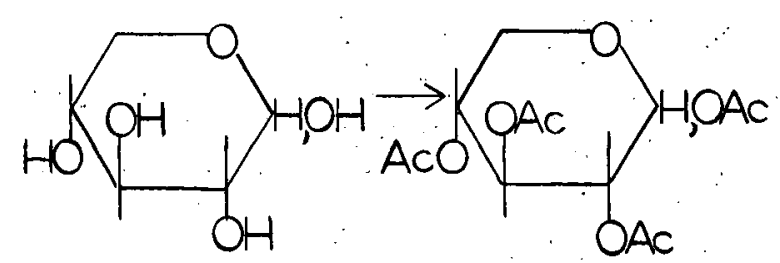

(41)

(42)

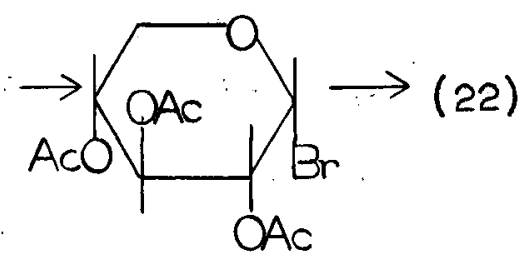

(43)

35

method for the preparation of this glycal $66^{\circ}$. Overend and co-workers 67 have pointed out the necessity of employing low temperatures $\left(-5\right.$ to $-10^{\circ}$ ) for the conversion of acetobromoaldoses to glycals, particularly in the pentose series, in order to minimise the simultaneous formation of saturated products $((25) \longrightarrow(26))$. In view of this, the yield of (22) reported by Gakhokidze ${ }^{68}$ ( $80 \%$ from (43)) was remarkably high as the reaction was performed at room temperature. 
Attempts to repeat this preparation, however, have resulted only in the isolation of 2,3,4-tri-0-acetyl-D-xylose in rather good yteld ${ }^{69}$.

In this work, the procedure of Helferich and coworkers ${ }^{45}$, in slightly modifled form, was used to prepare 3,4-di-0-acetyl-D-xylal. Conversion of (4I) to (22) was. thus carried through without isolation of the intermediate compounds (42) and (43). Consistently satisfactory results were obtained using this procedure providing the following precautions were observed:

(a) The entire preparation was carried through as quickly as possible, especially the final stage (43) $\longrightarrow(22)$. (b) The temperature of the zinc-acetic acid reduction mixture was not allowed to exceed $-10^{\circ}$; addition of solid carbon dioxide was effective in maintaining a low temperature, both during the course of the reaction and the subsequent filtration.

(c) Normaliy, removal of zinc by filtration is extremely slow, and as some rise in temperature is inevitable during this stage, undue delay is likely to lead to reduced ylelds. It was found that filtration could be greatly speeded by adding Celite to the reaction mixture, and spreading a layer of Celite on the fler paper before filtration. (c) The crude syrupy product obtained by chloroform extraction of the flltrate was distilled without delay. The 
product thus obtained crystallised spontaneously, and suffered no apparent decomposition when stored for several months in the refrigerator.

Typicaliy, by this procedure, 3,4-di-0-acetyl-Dxylal (22) was obtained from $\stackrel{D}{=}-x y l o s e ~(41)$ in an overall yleld of about $60 \%$. The purity of the product obtained by distillation was readily demonstrated by thin layer chromatography, acetylated glycals being considerably more moblle on silica gel than other components present in a crude preparation. The most characteristic region of infrared absorption of (22) is a sharp, falrly strong band at $1640 \mathrm{~cm}^{-1}$, assignable to the $\mathrm{C}=\mathrm{C}$ stretching vibration. The presence or absence of this band is therefore of use in following the course of reactions involving additions to the double bond.

(b) Reaction Conditions

Sultable conditions for the reaction of acetylated glycals with carbon monoxide, hydrogen and dicobalt octacarbonyl have been well established as a result of previous work ${ }^{64}$. The early experiments of Adkins and Kresk 9 sultably adapted the oxo reaction to a laboratory scale, and in general little deviation from these conditions is observed in the experiments of subsequent workers. However, in the case of the reaction of acetylated glycals, 
lower temperatures than are often customify have been used. Whereas at $130^{\circ}$ glycals are largely converted to alcohols by hydroxymethylation (equation $\underline{2}$ ), with the majority of olefins aldehydes are formed predominantly at this temperature, and appreclable alcohol formation is obtained only when the temperature is raised by $50^{\circ}$ or more. The most rapid reaction rate is obtained when the ratio of hydrogen to carbon monoxide is high ${ }^{12}$; the limiting factor would appear to be the requirement that the partial pressure of carbon monoxide is sufficientiy high to prevent decomposition of the catalyst ${ }^{2}$. In practice, 3,4,6-tri-0-acetyl-D-galactal (40) has been observed to react normally with carbon monoxide and hydrogen when the Initial partial pressures of the two gases were 200 and 1700 p.s.1. respectively 63 . For the hydroxymethylation of 3,4-d1-0-acetyl-D-xylal, a carbon monoxide to hydrogen ratio of $1: 5$ was employed, at a total Initial pressure of about 3000 p.s.1.

(c) Removal of Catalyst

Wender ${ }^{70}$ has described various methods for the removal of dicobalt octacarbonyl catalyst from oxo reaction products. When the isolation of aldehydes is not required, the preferred method is to replace unreacted synthes 1 gas with hydrogen under pressure, when, on heating, the octacarbonyl is decomposed to metallic cobalt. Alternatively, 
dicobalt octacarbonyl may be destroyed by heating the mixture on a steam bath, or shaking with dilute sulphurict ac1d solution, until carbon monoxide is no longer evolved. In working with the products derived from the acetylated glycals we have found the most conventent method for separating reaction products from catalyst is by filtration through Florisil (a synthetic magnesia-silica gel absorbent); catalyst is eluted with petroleum ether, and reaction products are subsequently eluted with a more polar solvent, such as a $9: 1(\mathrm{v} / \mathrm{v})$ mixture of benzene and ethanol.

(11) Fractionation and Characterisation of Reaction Products

(a) Chromatographic Separation of Products

Evidence that the anticipated hydroxymethylation of the double bond of 3,4-d1-0-acetyl-D-xylal had occurred was provided by the infrared spectrum of the catalyst-free product 1solated from the reaction: the strong absorption at $1640 \mathrm{~cm}^{-1}$ characteristic of the glycal double bond had disappeared, and a band of moderate intensity had appeared In the $3400 \mathrm{~cm}^{-1}$ region (OH stretching). Thillayer chromatography showed the presence of a mixture with two major components which were not well resolved, and indicated that little would be gained in attempting to fractionate the mixture as such. Attempted fractionation by gas-11quid partition chromatography ${ }^{71}$, following complete acetylation 
of free primary hydroxyl groups, gave one zone on a column of $20 \%$ Silicone G.E.S.F. 96 on firebrick at $190^{\circ}$, which was shown to be a mixture of two components by thin layer chromatography. Successful separation of the mixture of products from the oxo reaction was subsequently effected by deacetylation, and chromatography of the resulting mixture of polyols.

Following deacetylation by sodium methox'de in methanol 72 , and removal of sodium ions with Amberlite $\mathrm{IR}-120\left(\mathrm{H}^{+}\right)$ cation exchange resin, a syrupy product was obtained, whose infrared spectrum confirmed the complete removal of $\underline{0}$-acetyl groups, and whlch showed a strong, broad hydroxyl band. Preliminary examination by descending paper chromatography revealed that the deacetylated product comprised mainly two components, detectable on spraying with periodateSchiff reagent 73 with this spray reagent, compounds having an $\alpha$-glycol group show as purple spots on a white background, by virtue of their oxidation by periodate to a dialdehyde, which restores the colour of the Schiff reagent. After development for about 40 hours, using a solvent system of water-saturated 1-butanol contalning 5\% ethanol, the two major components of the mixture were sufflclently far apart to enable their separation to be effected on a preparative scale. A preparative separation of the two components of the polyol mixture was carried out by applying the material, in methanol solution, to several 
large sheets of Whatman's No. I paper prepared for descending chromatography. The progress of the separation was followed by detecting the positions of the zones on control chromatograms which were developed in the same tank, and was allowed to continue until the two components of the mixture were near the bottom edge of the sheets, thereby achieving maximum separation. Three narrow test strips were cut from each large sheet and sprayed with the zone-locating reagent 73 in order to ensure that the position of each zone was determined accurately; the material so lost represented from 5 to $10 \%$ of each component. The zones thus located were exhaustively extracted with aqueous methanol; the two fractions thus isolated, both inftially in the form of syrups, were found to be free from contamination by the other on rechromatography of a portion on paper.

Of the two chromatographically pure fractions resulting from the above separation, the faster moving component will be designated Fraction $I$, and the slower, Fraction II.

(b) Characterisation of Fractions I and II

From an amount of $400 \mathrm{mg}$ of the mixture obtained on deacetylation of the oxo product, $150 \mathrm{mg}$ of Fraction I and $180 \mathrm{mg}$ of Fraction II were pecovered from the chromatograms. Allowing for a loss of approximately $10 \%$ of the material 
Inftially applied (as a result of zone location); the combined fractions therefore represented about $90 \%$ of the $\mathrm{mix}-$ ture.

$\begin{array}{llcc} & \frac{R_{F}^{(a)}}{R^{(a)}}[\alpha]_{D}^{20^{(b)}} & \text { m.p. } \\ \text { Fraction I } & 0.47 & -13^{\circ} & 102^{\circ} \\ \text { Fraction II } & 0.41 & -44^{\circ} & -\end{array}$

(a) -in water-saturated 1-butanol + 5\% ethanol, at room temperature

(b) -1 water

Fraction I, after crystallisation to constant meltIng point, gave an elemental analysis corresponding to an empirical formula of $\mathrm{C}_{6} \mathrm{H}_{12} \mathrm{O}_{4}$. Acetylation of Fraction I with acetic anhydride-pyridine gave a syrup which could not be crystallised, although thin layer chromatography showed the homogeneity of the product. This fraction was characterised as the p-nitrobenzoyl derivative, m.p. $215^{\circ}$, $[\alpha]_{D}-50^{\circ}$. The elemental analysis of the crystaline derivative corresponded to the replacement of three hydrogens of Fraction I by three p-nitrobenzoyl groups.

Fraction II resisted attempts at crystalisation, but readily formed a crystalline derivative, m.p. $80-81^{\circ}$, $[\alpha]_{D}-41^{\circ}$, on acetylation with acetic anhydride-pyridine. This derivative gave an analysis corresponding to $\mathrm{C}_{12} \mathrm{H}_{18} \mathrm{O}_{7}$, 
the tri-o-acetyl derivative of Fraction II. In order to obtain Fraction II in a highly purified form, a portion of the crystalline acetate was deacetylated by the action of methanolic sodium methoxide and the product was carefully isolated in the usual way to give a syrup which did not crystallise, but which analysed satisfactorily for $\mathrm{C}_{6} \mathrm{H}_{12} \mathrm{O}_{4}$.

(iii) Identification of Fractions I and II

(a) Periodate Consumption

Information on the structure of a carbohydrate can be gained from a study of its reaction with periodate $10 n^{74}$. The chief analytical application of this reaction is in the determination of the number of adjacent hydroxyl groups in the molecule, as each $\alpha$-glycol group consumes one molecular proportion of perlodate, the resulting fragments being formaldehyde, formic acid or a substituted aldehyde according to the location of the particular group undergoing oxidation.
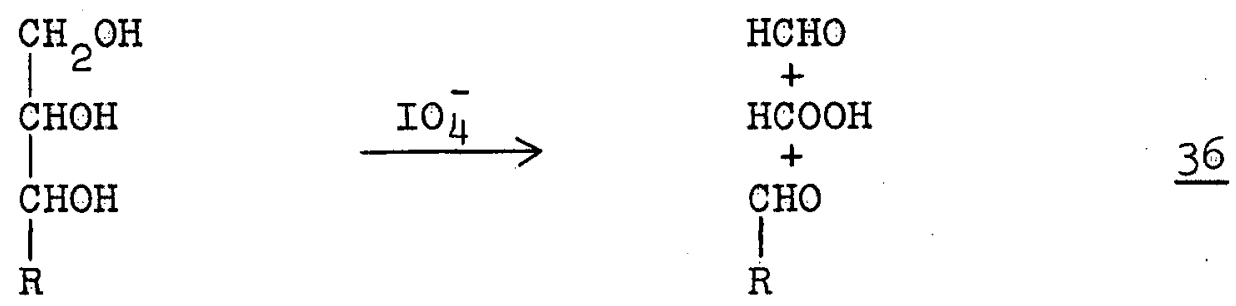

Other groups such as $\alpha$-hydroxyaldehydes, $\alpha$-hydroxyketones and $\alpha$-amino-alcohols are also cleaved by periodate. 
Varlous methods are in common use for following the reaction of vicinal hydroxyl groups with periodate 1on ${ }^{75}$, usually involving titrimetric procedures and requiring the destruction of appreclable amounts of material. The spectrophotometric method of $\mathrm{Dix}$ on and $\mathrm{Lipk1n^{76 }}{ }^{6}$, requiring only $10^{-8}$ to $10^{-6}$ mole of sample, appeared to be eminently suitable for determining the number of vicinal hydroxyl groups In Fractions I and II, limited amounts of which were available. These workers found that consumption of perlodate may be followed spectrophotometricaliy at $223 \mathrm{~m} \mu$, at which wavelength its absorption is at a maximum 77 . No inaccuracies were introduced by the relatively small absorption of lodate ion in this region, nor by absorption of unconjugated carbonyl compounds formed during the reaction.

Following this procedure, the decrease in absorbance at $223 \mathrm{~m} \mu$ of a solution of Fraction $I\left(0.439 \times 10^{-4} \mathrm{M}\right.$ on the basis of a molecular weight of 200) containing an excess of sodium periodate $\left(0.942 \times 10^{-4} \mathrm{M}\right)$ was measured over a period of several hours. Each reading (A) on the Beckman Model DU Spectrophotometer was accompanied by a reading (B) of a control solution $0.942 \times 10^{-4} \mathrm{M}$ with respect to periodate ion, and also of a solution containing $0.439 \times 10^{-4} \mathrm{M}$ of Fraction I (C). Thus $(B+C)-A$ was a measure of the decrease In absorbance due to consumption of periodate ion by Fraction I. Then $\frac{(B+C)-A}{C_{O}}$ was the fraction of the known amount of 
added periodate which was consumed by the carbohydrate at any time, where $C_{0}$ was the measured absorbance of the per10date solution at zero time (this changes slowly with time because of variations in $\mathrm{pH}$, temperature ${ }^{77}$ ). It was found that $A$ reached a constant value equivalent to the consumption of 0.90 mole of periodate ion per mole of Fraction $I$. A similar series of measurements with Fraction II gave a value of 0.95 moles of perlodate per mole of substrate. With due allowance for the micro scale of these analyses, these results therefore showed the presence of two vicinal hydroxyl groups in each compound.

\section{(b) Structures of Fractions I and II}

Thus for, the information obtained on Fractions I and II Indicated that both contained three hydroxyl groups; two of which were vicinally situated. On this evidence, and from a consideration of the likely mode of addition of carbon monoxide and hydrogen to 3,4-di-0-acetyl-D-xylal (22) It could be assumed that the reaction had followed the expected course, and a hydroxymethyl group had added to the double bond. On this basis, four isomers..(44) to (47) were possible, all of which contain two vicinal secondary

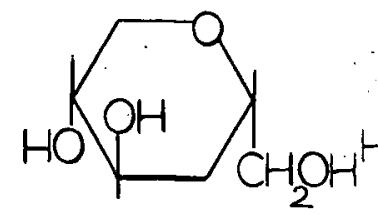

(44)

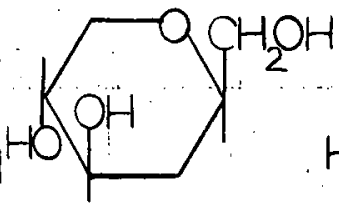

(45)

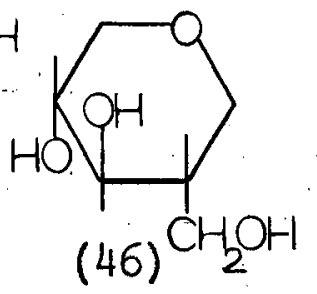

(46)

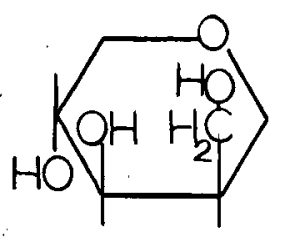

(47) 
hydroxyl groups in addition to one primary hydroxyl group, and therefore could not be distingulshed on the avallable evidence. Structures (44) and (45), resulting from the addition of a hydroxymethyl group at C-l of the giycal, are 1,5-anhydro-4-deoxy-hexitols, differing in the configuration of carbon 5 ; in accordance with accepted nomenclature ${ }^{78}$, (44) and (45) are preferably drawn in the following way

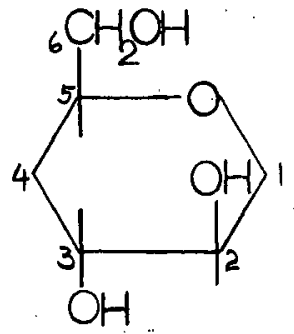

(44)

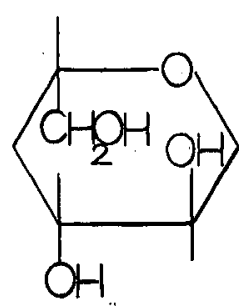

(45)

Hydroxymethylation at $c-2$ of the glycal would give either of the branched-chain 1somers (46) and (47), which are 1,5-anhydro-2-deoxy-2-hydroxymethyl-pentitols, differing in configuration of the side chain at $c-2$.

It was possible to distinguish between the straight chain and branched chain structures merely from a consideration of the resonance positions and relative intensities of the proton signals observed in the nuclear magnetic resonance spectra of Fractions I and II, measured in deuterlum oxide solution. In this solvent, hydroxylic protons are rapidiy exchanged and are resolved into one sharp H-O-D signal; the 
spectrum is thereby simplified. Consideration of structures (44) to (47) shows that in both straight chain and branched chain isomers are present (a) methylene protons $\alpha$ to an oxygen, $\mathrm{C}-\mathrm{CH}_{\mathrm{Q}}-\mathrm{O}_{-}$, and (b) a methine proton $\alpha$ to an oxygen function, ot-o-. In the straight chain 1somers (44) and (45), a methylene group is also present which is flanked by two carbon atoms; (c) $\mathrm{C}-\mathrm{CH}_{2}-\mathrm{C}$, whereas in the branched chain isomers (46) and (47) a tertiary hydrogen, rather than a methylene group, is present; (d) $\mathrm{C}_{-} \mathrm{CH}-\mathrm{C}$. It is fundamental to nuclear magnetic resonance spectroscopy that the resonance position, or chemical shift, of a proton depends upon its precise chemical environment. Ample evidence is avallable to indicate with certainty that protons of types (a) and (b) above will resonate at a lower magnetic field than will type (c) and (d) protons, because the inductive effect of the adjacent oxygen atom will reduce the electron density around these protons. Jackman 79 tabulates typlcal values for methylene and methine protons with an $\propto$-oxygen function as lying between 6.15 and $6.60 \tau(\delta=3.85-3.40$ ppm), whereas the corresponding protons in saturated hydrocarbons resonate around $8.5 \tau$ ( $1.5 \mathrm{ppm})$, with deshielding from $\beta$-substituents resulting in a comparatively minor shift to lower fleld.

Consequentiy, in $D_{2} 0$ solution, it was anticipated that the non-hydroxylic hydrogens of the straight chain isomers (44) and (45) would exhibit two types of signals: a group 
at lower fleld of relative intensity 7 , corresponding to type (a) and (b) protons, and a higher fleld group of relative intensity 2 , corresponding to the two methylene protons, (c) On the other hand, with the branched chain isomers (46) and (47) the lower field group would have a relative intensity of 8 , whereas the single tertiary hydrogen (d) would resonate at higher fleld with an intensity of 1. The observed n.m.r. spectra of Fractions I and II both showed the anticipated separation of signals into lower and higher field groups, (In addition to the single $H-O-D$ peak at $\delta=4.73 \mathrm{ppm}$ ). In both cases the area enclosed by the lower fleld group of signals $(\delta=2.9-4.2 \mathrm{ppm})$ was 3.5 times that of the group at higher field $(\delta=1.1-2.2 \mathrm{ppm})$. This clearly demonstrated that both Fractions I and II were isomeric 1,5-anhydro-4deoxy-hexitols (structures (44) and (45)). Additional information regarding the stereochemistry of these compounds can be derived from a consideration of the multiplicities of the higher fleld signals; this aspect is discussed in more detail later.

Further confirmation that Fractions I and II were isomeric 1,5-anhydro-4-deoxy-hexitols was based on the following argument. Periodate cleavage of the $\propto$-glycol group in all four structures (44) - (47) would furnish a dialdehyde which on reduction would give a structure having three primay hydroxyl groups and an $\epsilon$ ther Iinkage. However, whereas 
from (44) and (45) a pasr of enantioneric triol ethers (48) and (49) would be obtained, each having one centre of asymmetry (*), the same reactions applied to either branched chain isomers, (46) or (4\%), wolld result in the same optically-inactive triol ether (50) being formed.

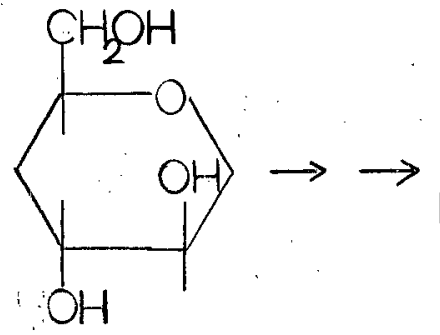

(44)

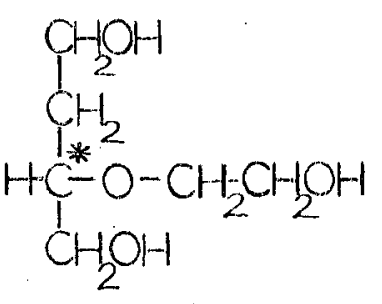

(48)

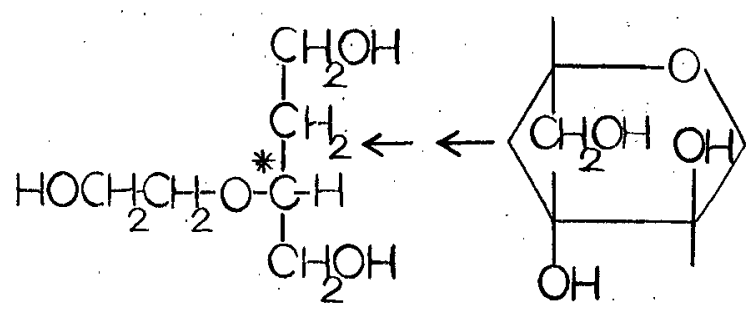

(49)

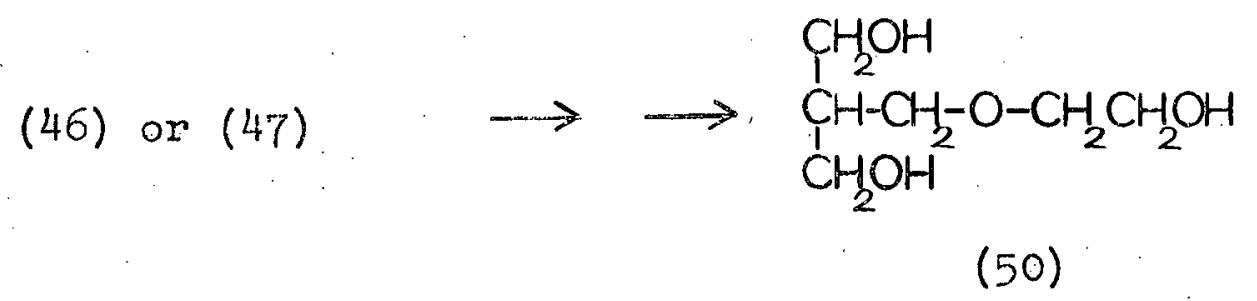

Both Fractions I and II were separately treated in this way, according to a procedure similar to that described by von Rudlofi and co-workers 80 . A sample of each polyol was oxidised with a $50 \%$ excess of perlodic acid until the optical rotations of the solutions were constant. After neutralising with barium earbonate the solutions were treated with an aqueous solution of sodium borohydride; cations were

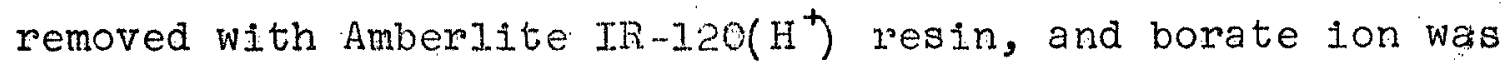


81

volatalised as the methyl ester ${ }^{81}$. The product in both cases was a syrup.

The triol ether obtained from Fraction I had $[\alpha]_{D}-19^{\circ}$, and that from Fraction II had $[\alpha]_{D}+17^{\circ}$. Both products had Identical n.m.r. spectra, measured in deuterium oxide solution, with a group of signals $\delta=3.5-3.9 \mathrm{ppm}$, and a higher field group at $\delta=1.5-2.0 \mathrm{ppm}$, the relative areas of the two groups being in the rat1o of $9: 2$. Hence the products resulting from the periodate oxidation and sodium borohydride reduction of the two polyols were clearly the enantiomeric $\underline{=}-(48)$ and $\underline{L}$ - (49) forms of 2-deoxy-3-0-(2-hydroxyethyl)glycero-tetritol.

It was noted that, in the course of an investigation of the products resulting from the hydrogenolysis of methyl $\alpha-D-g l u c o p y r a n o s i d e(51)$ under conditions of high temperature and pressure, and in the presence of a copper chromite catalyst, von Rudloff and co-workers 80 had isolated, in addition to several other products, a 2-hydroxymethy1-4,5-dihydroxytetrahydropyran (52); (considered as a carbohydrate, (52) is a 1,5-anhydro-4: deoxy-hexitol)

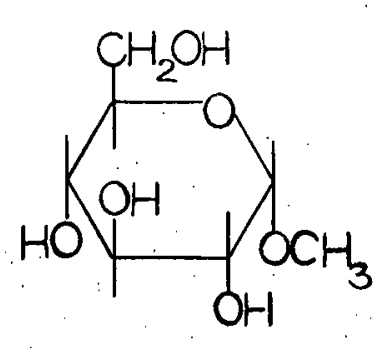

(51)
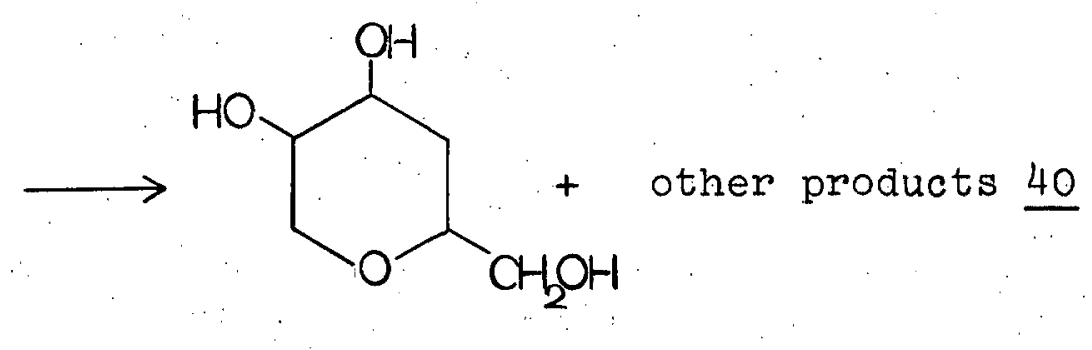
The molecular structure of (52) (which later proved to be a mixture of stereo1somers ${ }^{82}$ ) was demonstrated by perlodate oxidation to a dialdehyde which was then reduced with sodium borohydride to an open chain triol ether (53), characterised as the triacetate. The structuae of the triol ether was proved in two ways: (a) ethyl lodoacetate was condensed with diethyl I-malate in the presence of sodium, and the resulting triester (54) was reduced with I1thium aluminum hydride<smiles>CCOC(=O)CC(COC(=O)OCC)OC(CC(=O)OCC)C(=O)OCC</smiles>

(b) cleavage of the triol ether with boron trichloride 83 in acetic anhydride and subsequent deacetylation gave ethanediol and 1,2,4-butanetriol<smiles>OCCC(CO)OCCC(CCO)C(O)CO</smiles> 
The product (52) from the hydrogenolysis of (51) was further investigated by Gorin 82 , who fractionated the material by cellulose column chromatography into two 1someric 1,5-anhydro-4-deoxy-hexitols. Periodate oxidation and borohydride reduction of both components afforded the same triol ether, which demonstrated that the configuration of the hydroxymethyl group was the same in each case, and therefore the two compounds differed in the configuration of the vicinal hydroxyls on the ring. The triol ethers obtained by Gorin were levorotatory $\left([\alpha]_{D}-20^{\circ},-16^{\circ}\right)$, and were characterized as the tris-p-nitrobenzoates, melting points $103-104^{\circ}, 98-102^{\circ}$, specific rotations $-26^{\circ},-22^{\circ}$.

The enantiomeric triol ethers obtained from Fractions I and II, which for the sake of convenience will be designated as compounds III and IV respectively, were converted to p-nitrobenzoyl derivatives according to the procedure of Gorin 82 . The levorotatory triol ether III from Fraction I gave a crystalline derivative m.p. $102-103^{\circ},[\alpha]_{D}-28^{\circ}$, which did not depress the melting point of Gorin's tris-p-nitrobenzoate 84 , and the dextrorotatory triol ether IV, from Fretion II, furnished the enantiomeric derivative, m.p. 102-103 ${ }^{\circ}$, with an equal and opposite specific rotation. As the previous work of von Rudloff and co-workers ${ }^{80}$ had clearly demonstrated the positions of attachment of the hydroxymethyl group and the two secondary hydroxyl groups to the tetrahydropyran 
ring of (52), these results provided additionil and conclusive proof that Fractions I and II were 1someric 1,5anhydro-4-deoxy-hexitols.

One implication of the demonstrated relationship between the two isomeric anhydrodeoxyhexitols derived from methyl $\propto$-D-glucopyranoside (5I) and Fraction $I$ was that, assuming no inversion of configuration occurred at c-5 of . the glycopyranoside ring of (51) during the hydrogenolysis reaction, then Fraction I must have the $\stackrel{D}{=}$ configuration at C-5 (44), and consequentiy Fraction II must have the Iconfiguration at this centre (45). However, such an assumption was not considered sufficiently justifiable without - further confirmation, for two reasons:

1. The hydrogenolysis reaction of von Rudloff and co-workers 80 was carried out under extremely vigorous conditions, and in general it has been found that these reactions are accompanied by considerable configurational changes 85,86 .

2. Assuming the secondary hydroxyl groups of Fractions I and II were unchanged in configuration as a result of the oxo reaction, then on the basis of the evidence described above, of the two possible 1,5-anhydro-4-deoxy-hexitols, one of the two fractions must be 1,5-anhydro-4-deoxy-D-arabinohexitol (44), and the other must be the corresponding L-xy101somer (45). Both the anhydrodeoxyhexitols 1solated by for ${ }^{82}$ 

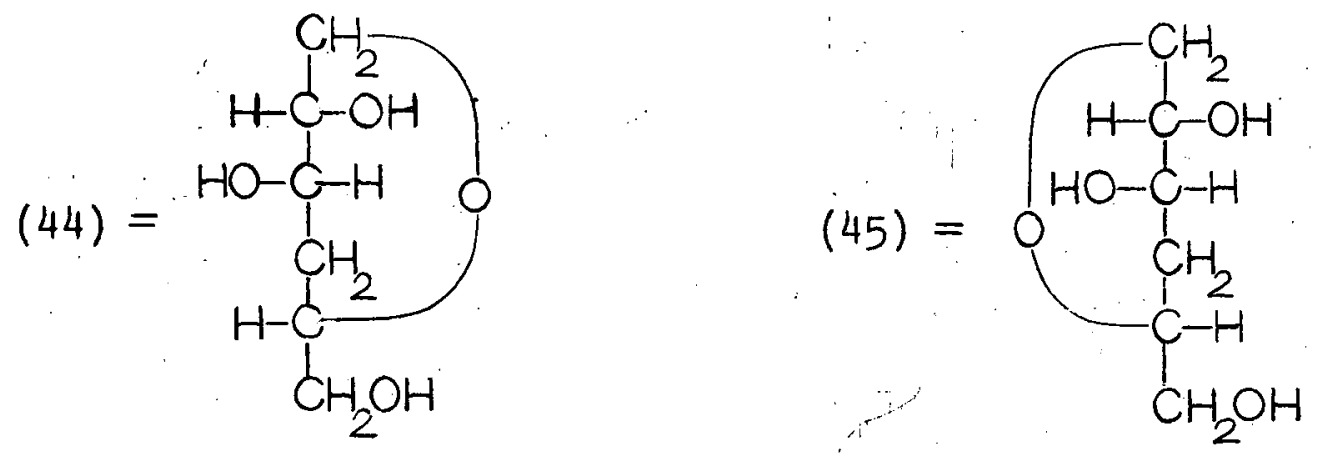

were assumed to be of the D-series as they were derived from a D-glucopyranoside (51), and on the basis of certain evidence, which w1ll be discussed in more detail below, wee assigned the D-arabino-(44) and D-Iyxo-(60) configurations. However, the properties of the isomer which was considered to have the D-arabino-configuration (44) did not resemble those of elther Fraction I or Fraction II.

(iv) Configurations of Fractions I and II

(a) Stereochemistry at C-5

The stereochemistry at $\mathrm{C}-5$ of the 1,5-anhydro-4deoxy-hexitols. I and II was established with certainty once 1t was known which of the enantiomeric triol ethers III and IV was 2-deoxy-3-0-(2-hydroxyethyl)-D-glycero-tetr1tol (48), and which was the L-1somer (49). Attempts were made to obtain one of the optically-pure enantiomers by an unequivocal route; this was realised by the preparation of the L-1somer 
(49) from a structupe of known stereochemistry, 1,4-anhydro5-deoxy-D-arabino-hexitol (59), obtained from the known compound 3,6-anhydro-2-deoxy-D-1yxo-hexose (58) on reduction with sodium borohydride. The anhydrodeoxy-sugar (58) was obtained by the procedure of Foster and co-workers 87 from methyl 2-deoxy- $\propto$-D-galactopyranoside (55), which in turn.

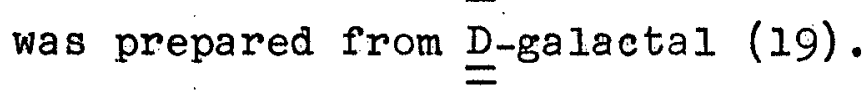

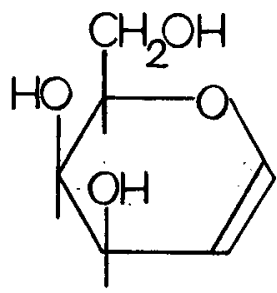

(19)

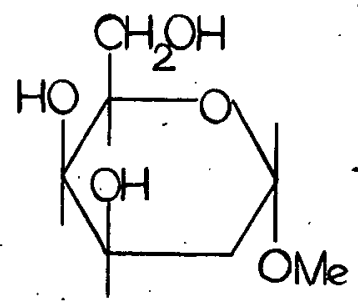

(55)

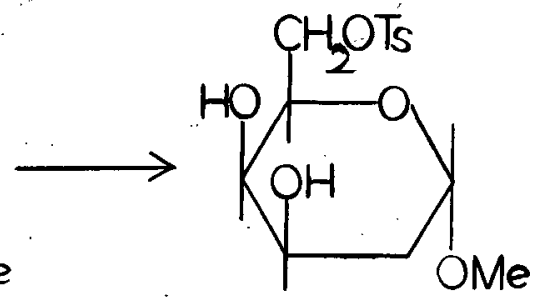

$(56)$

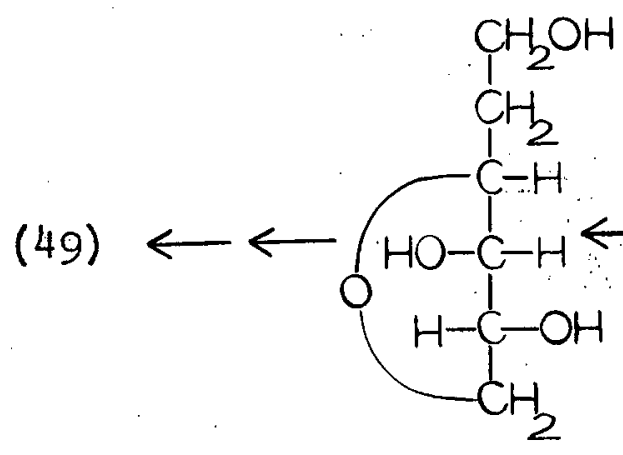

(59)

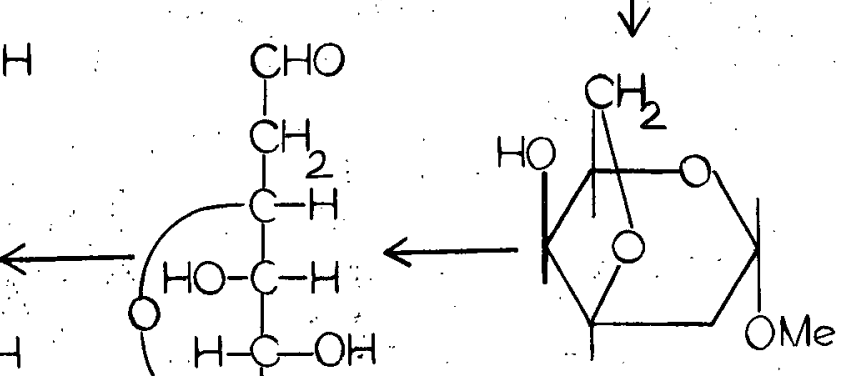

(57)

(58)

D-galacta1 (19) was obtained from 3,4,6-tri-0-acety1D-galactal (40) on deacetylation with methanolic sodium methoxide ${ }^{88}$; transesterification of $(40)$ (and other acetylated glycals ${ }^{89,66}$ ) under these conditions is unusually slow, taking 2 days for completion. D-galactal crystallised readily from 
the residue remalning after neutralisation and evaporation of solvent, upon extraction with ethyl acetate.

Preparations of methyl 2-deoxy-D-galactopyranoside (55) have been reported by Tamm and Reichstein ${ }^{90}$ and by Overend and co-workers ${ }^{42}$; in each case D-galactal (14) was first converted to 2-deoxy-D-galactose by the addition of water across the double bond in dilute sulphuric acid solution, and the sugar was then methylated in the presence of hydrogen chloride, the more stable $\propto$-glycoside (55) being isolated in crystalline form. The latter workers also observed the direct formation of (55) when D-galactal was treated with $3.3 \%$ methanollc hydrogen chloride and Foster, Overend and Stacey" ${ }^{91}$ obtalned the $\propto$-methyl glycoside on a qualitative scale when a solution of D-galactal in $0.4 \%$ methanolic hydrogen chloride was allowed to reach rotational equilibrium over 43 minutes. The one step conversion of D-galactal to methyl 2-deoxy- $\alpha$-D-galactopyranoside was used on a preparative scale in this work. The change in optical rotation of a solution of D-galactal (19) in methanol containing $0.3 \%$ hydrogen chloride was followed, and found to be constant after about 50 minutes. A syrup was 1solated from this reaction which crystalifiped readily on adding a small volume of acetone; the product was Identifled as (55) from 1ts melting point of $112-114^{\circ}$, (Overend and co-workers ${ }^{42}$ found $112-113^{\circ}$ ). 
The preparation of 3,6-anhydro-2-deoxy-D-1yxo-hexose (58) from (55) was carried out by the method of Foster and co-workers 87 ; these workers applied the known procedure of Haworth and co-workers 92 for the preparation of 3,6-anhydrosugars, Involving alkaline treatment of the 6-0-p-tolysulphonyl derivatives of the methyl glycosides, with formation of the corresponding methyl 3,6-anhydro-glycosides. Thus reaction of (55) with I molar equivalent of p-toluenesulphonyl chloride under conditions favourable for unimolar sulphonylation 93 gave the syrupy 6-0-p-tolylsulphonyl derivative (56), which on treatment with base was converted to methyl 3,6-anhydro-2-

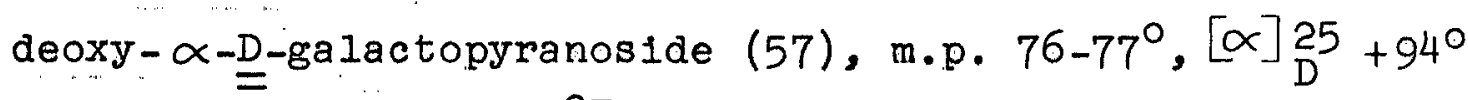
(Foster and co-workers 87 give m.p. $\left.80^{\circ},[\alpha]\right]_{D}^{15}+98^{\circ}$ ). As proof of the configuration of the triol ether (49) ultimately obtained from 1,4-anhydro-5-deoxy-D-arabino-hexitol (59) essentially depends upon a knowledge of the conflguration at $\mathrm{C}-3$ the anhydro-deoxy-galactoside (57), it is necessary to consider the evidence for the structure of this compound: (a) (57) was characterised as the crystalline 4-0-ptolysulphonyl derivative 87 .

(b) When the syrupy 6-0-p-tolylsulphonyl derivative (56), from which (57) was prepared, was heated with sodium lodide In acetone solution, an equivalent amount of sodium ptoluenesulphonate was obtained, evidence that the tosyloxy group of (56) is at the primary (c-6) position 94 .

(c) The mechanism of formation of anhydro-sugars in basic 
medium is well established, and has been reviewed by peat ${ }^{95}$. 3,6-Anhydro-sugars are of the hydrofuranol type, in which the anhydro-ring is 5-membered; both this type and the well known ethylene oxide ( 3 -membered) anhydrides are often. prepared by treatment of a tosyl derivative with base. The reaction involves an intramolecular exchange of anions at a carbonium ion, whereby a tosyloxy group is replaced by anionic oxygen, and is most clearly illustrated with reference to the ethylene oxide type of anhydride. Thus anhydride
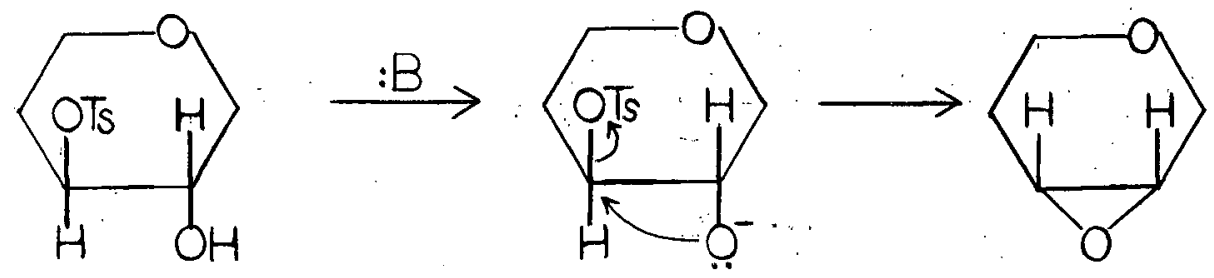

44

formation involves inversion of configuration at the carbon atóm bearing the potential leaving group (which may also be halogen or other mineral acid ester group), but no inversion of configuration occurs at the hydroxylic carbon. It follows that the displacing ion must have a trans arrangement with respect to the leaving group before an anhydride ring can form. The same principle holds in the formation of 3,6-anhydrides as a result of replacement of a tosyloxy group at $\mathrm{c}-6$ by nucleophilic oxygen at $\mathrm{c}-3$. As $\mathrm{c}-6$ is not asymmetric no inversion of configuration is apparent; however, 
the formation still requires a trans disposition of the entering and leaving groups. For steric reasons the free hydroxyl group at $\mathrm{C}-3$ must be on the same side of the sugar ring as the side chain, C-6. Clearly, therefore, formation of 3,6-anhydro-sugars under these conditions results in no

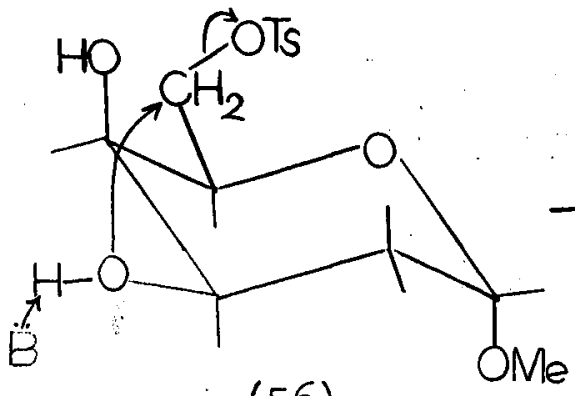

(56)

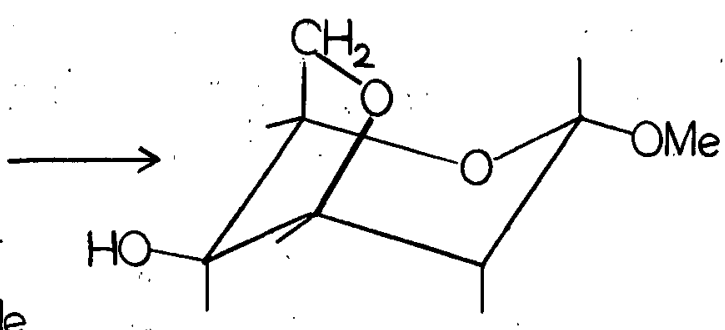

(57)

45

configurational inversion at $\mathrm{C}-3$, and (59), obtained from (58) on sodium borohydride reduction, must have the Lconfiguration at $\mathrm{C}-4$.

$$
\text { 3,6-Anhydro-2-deoxy-D-1yxo-hexose (58), was obtained, }
$$
on treatment of the anhydrodeoxygalactoside (57) with dilute acid at room temperature, as a syrup, $[x]_{D}+25^{\circ}$ (reported $\left.[\propto]_{D}+24^{87}\right)$. Foster and co-workers note that the anhydrosugar (58) exists in the aldehydo-form, readily restoring the colour to Schiff reagent, and a carbonyl band at about $1715 \mathrm{~cm}^{-1}$ was observed in the infrared spectrum of the product. In this respect (58) differs from the corresponding derivatives of D-glucose and D-mannose, which exist in the furanose form, an arrangement of two c1s-fused 5-membered 
rings being in a less-strained state than a 3,6-anhydride bridge across a pyranose ring. Such an arrangement is not possible in 3,6-anhydro-D-galactose and 1ts 2-deoxy-derivative (58), because of the orientation of the hydroxyl group at c-4.

Reduction of (58) with sodium borohydride gave 1,4anhydro-5-deoxy-D-arabino-hexitol (59), as evidenced by the disappearance of carbonyl absorption in the infrared. The anhydrodeoxyhexitol (59), which does not appear to have been reported previously, had $[\alpha]]_{\mathrm{D}}^{25}+21^{\circ}$, and was a syrup. It was characterised as the tris-p-nitrobenzoyl derivative, m.p. $159-160^{\circ},[\alpha]_{D}^{22}-96^{\circ}$. (59) was oxidised with excess 0.1 perlodic acid, under conditions similar to those employed for the cleavage of Fractions I and II. By comparison with these previous reactions, the oxidation of (59) was slow, as would be anticipated for a trans $\alpha-8 l y c o l$ group on a 5-membered ring . Sodium borohydride reduction of the resulting dialdehyde then gave 2-deoxy-3-0-(2-hydroxyethy 1)L-glycero-tetritol (49); this had $[\alpha]_{D}+17^{\circ}$, and formed a tris-p-nitrobenzoate, m.p. $101-102^{\circ}$, [ot $]_{D}+27^{\circ}$, whose melting point was... undepressed on admixture with the $\underline{p}$ nitrobenzoate of the triolether IV obtained from Fraction II.

Thus, Fraction II must have the L-configuration at $C-5$, and Fraction $I$, which on cleavage and reduction afforded 
the enantfomeric triol ether III, must have the D-configuration at $\mathrm{C}-5$. These results confirmed Gorin's assumption 82 that no inversion occurred at. C-5 of methyl $\alpha-D-g l u c o p y r a n o s i d e$ (51) during the course of the hydrogenolysis of this compound 80 , and therefore both polyols isolated from this reaction were of the D-series.

(b) Configurations of Secondary Hydroxyls of Fractions I and II

An element of disagreement with Gorin's results still prevented a completely unequivocal assignment of the Darabino-(44) and L-xylo-(45) configurations to the two anhydrodeoxyhexitols, Fractions I and II respectively. Gorin 82 found that his two polyols, which for the sake of convenience will be designated as $X$ and $Y$, consumed lead tetraacetate at distinctly different rates, $X$ being oxidised four times faster than $Y$ in the initial stages. As both polyols were otherwise structurally similar, this was taken as evidence 96 that $X$ contained a $\underline{c i s}$, and $Y$ a trans $\propto-$-glycol group. Assuming that the configuration at $C-5$ was $D$ in both cases (an assumption justified by our results), a total of four possible 1somers, two $\underline{\mathrm{c}} \mathrm{s}$ and two trans, was then considered.

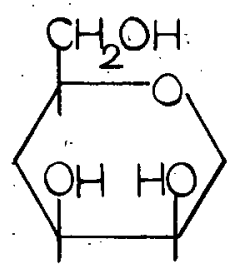

$\stackrel{D}{=}-\underline{y x o}$ (60)

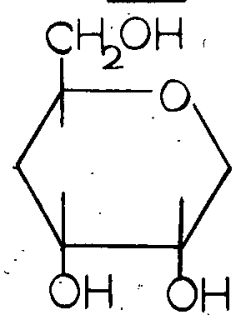

D-rivo

(61)

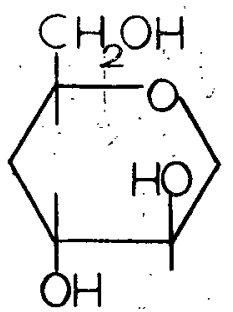

D-arabino

(44)

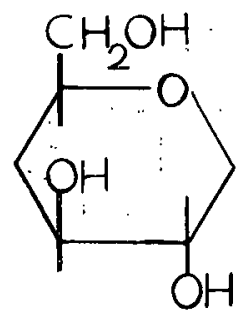

$D-x y 10$ (62) 
For polyols $X$ and $Y$, Gorin found specific rotations of $-50^{\circ}$ and $+19^{\circ}$ respectively. These values were compared with those calculated by application of the principle of optical superposition, first postulated by van't Hoff and later applied to carbohydrates by Hudson 97 in the form of his Isorotation Rules. For each of the four possible isomeric anhydrodeoxyhexitols $(44),(60),(61)$ and $(62)$, a value for the molecular rotation was derived by reference to a pair of structures of known molecular rotations, whose individual asymmetric centres "cancelled out" except for those which were also present in the anhydrodeoxyhexitol. This is best 1llustrated by two examples, selected from the six tabulated by Gorin, which pertain to the D-Iyxo-(60) and D-arabino-(44) 1somers.

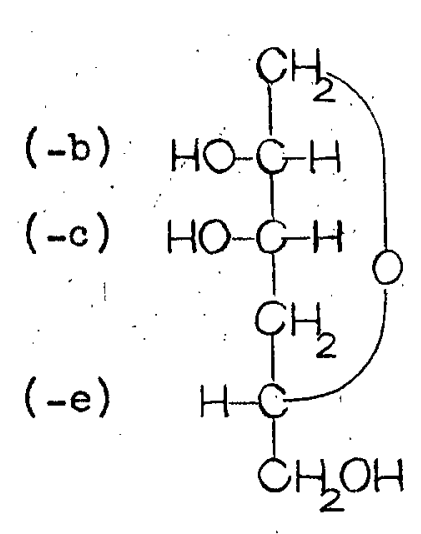

(60)

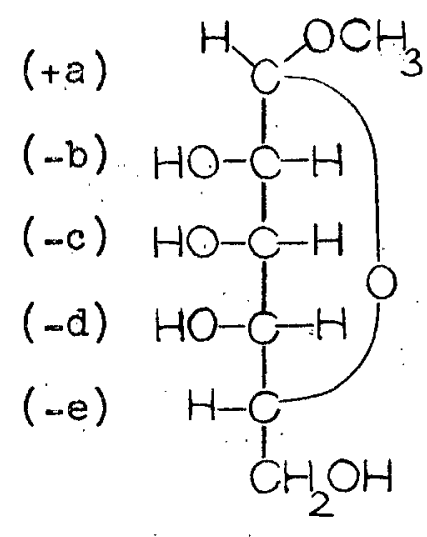

(63)

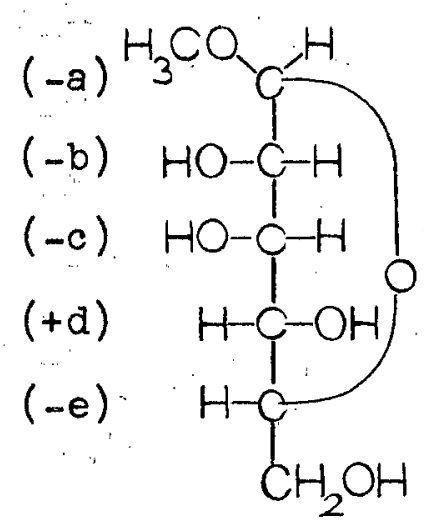

(64)

Let the three individual centres of asymmetry in 1,5-anhydro-4-deoxy-D-1yxo-hexitol (60) at carbons 2,3 and 
5, be assigned individual contributions to the total molecular rotation of $-b,-c$ and $-e$. The corresponding contributions of the five asymmetric centres of methyl $\alpha-D-$ tallopyranoside (63), whose molecular rotation, $[\mathrm{M}]_{\mathrm{T}}$, is $+4,070^{\circ}$, are $+a,-b,-c,-d$ and $-e$, and in methyl $\beta-D-$ mannopyranoside $(64),\left([\mathrm{M}]_{\mathrm{M}}=-13,390^{\circ}\right)$, are $-\mathrm{a},-\mathrm{b},-\mathrm{c}$, $+d$ and $-e$. Therefore $[\mathrm{M}]_{\mathrm{T}}+[\mathrm{M}]_{\mathrm{M}}=2(-\mathrm{b}-\mathrm{c}-\mathrm{e})$, so that $[\mathrm{M}]_{\ell}$, the calculated molecular rotation of the D-lyxo-isomer, is $[\mathrm{M}]_{\mathrm{T}}+[\mathrm{M}]_{\mathrm{M}} / 2=-4,660^{\circ}$, whence the calculated specific rotation, $[\mathrm{M}] / \mathrm{N}$, is $-31^{\circ}$.

In a similar manner the molecular rotation $[\mathrm{M}]_{a}$ of the D-arabino-isomer (44) was obtained by halving the sum of the molecular rotations of methy $\alpha$-D-altropyranoside (65) ( $\left.[\mathrm{M}]_{\mathrm{A}}=24,420^{\circ}\right)$ and of methyl $\beta-$ - $=$-1do pyranoside (66) $\left([\mathrm{M}]_{I}=-18,430^{\circ}\right)$. This gave a value of $+20^{\circ}$ for . the specific rotation of the Darabino-isomer (44).

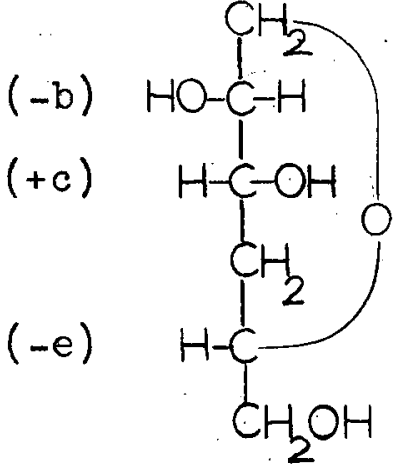

(44) $(+a)$

$(-b)$

$(+c)$

$(+d)$

(-e)

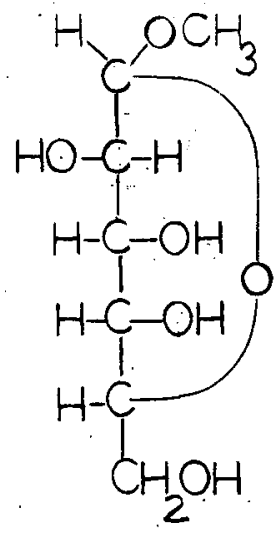

(65) $(-a)$

$(-b)$

$(+c)$

$(-d)$

(-e)

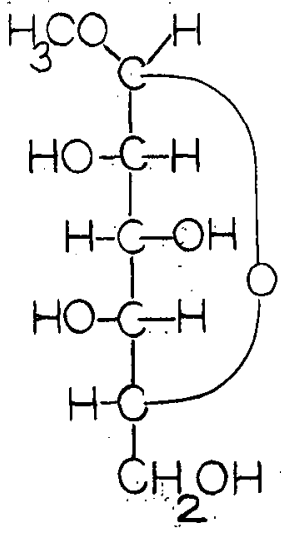

(66) 
Calculated values for the D-ribo-(61) and D-xylo-(62) 1somers, from the known molecular rotations of various other methyl hexopyranosides and 1,5-anhydrohexitols, were as tabulated below.

\begin{tabular}{|c|c|c|c|c|c|}
\hline \multicolumn{2}{|c|}{ Observed $[\alpha]_{D}$} & \multicolumn{4}{|c|}{$\begin{array}{c}\text { Calculated }[Q]_{D} \text { of 1someric } 1,5 \text {-anhydro- } \\
4 \text {-deoxy-D-hexitols }\end{array}$} \\
\hline $\mathrm{X}$ & $Y$ & D-r1bo $(61)$ & $\underline{D}-1 \mathrm{yx} \circ(60)$ & D-xyzo(62) & D-arabino (44) \\
\hline$-50^{\circ}$ & $19^{\circ}$ & $46^{\circ}$ & $-31^{\circ}$ & $106^{\circ}$ & \\
\hline & & & $-34^{\circ}$ & $67^{\circ}$ & \\
\hline
\end{tabular}

Comparisons of the observed rotations of polyols $X$ and $Y$ with those calculated for the four possible stereolsomers then led to the conclusion that polyol $x$ was probably 1,5anhydro-4-deoxy-D-1yxo-hexitol (60), and that $Y$ was probably the D-arabino-isomer (44). Th1s conclusion therefore disagreed with the assignment of the D-arabino-configuration to Fraction $I$, which had a specific rotation of $-13^{\circ}$. Furthermore, Gorin's polyol $\mathrm{Y}$ was characterised as the tris-p-nitrobenzoate, m.p. 115-119,$[\alpha]_{D} 59^{\circ}$, whereas Fraction I gave a p-nitrobenzoyl derivative with m.p. $215^{\circ}$ and $[\alpha]_{D}-50^{\circ}$.

It was then necessary to consider the possibility that configurational inversior had occurred during the course of the oxo reaction of 3,4-d1-0-acetyl-D-xylal. Certain circumstancial evidence can be mentioned which indicated that the D-threo-configuration of the six-membered ring was retalned during this reaction. Thus when 3,4-di-0-acetyl- 
D-xylal was reacted with only 2 moles of synthesis gas, a quantity of unchanged glycal was 1solated. The trans-arrangement of the $\alpha-g$-gcol system in Fractions I and II was indicated by the fact that neither compound formed a derivative with acetone, as it is known that a cis configuration is a prerequisite of isopropylidene acetal formation.

Some exploratory experiments were carried out with a view to synthesising the D-arabino-(44) and L-xylo-(45) isomers of 1,5-anhydro-4-deoxy-hexitol by an alternative route from 3,4-d1-0-acety1-D-xylal, whereby the possibility of ring inversions was absent. The recently reported preparation by Coxon and Fletcher 98 of a 2,6-anhydro-heptitol by reduction, followed by deamination of $2,3,4,6$-tetra-0acetyl- $\beta$-D-glucopyranosyl cyanide suggested the possibility of employing a similar sequence of reactions subsequent to the introduction of a cyano-group at C-I of the glycal. It is claimed 99 that hydrogen cyanide reacts with 2-alkoxy derivatives of 2,3-dihydro-4H-pyran (1), in the presence of a basic catalyst, with formation of 6-cyano-2-alkoxytetrahydropyrans. However no reaction was observed over several hours when 3,4-d1-0-acetyl-D-xylal (22) was dissolved in anhydrous hydrogen cyanide 100 in the presence of sodium cyanide; apparently the double bond of glycals is insufficiently activated for this addition to take place ${ }^{101}$. It was noted that 2-cyano-tetrahydropyran has been prepared 
by the addition of hydrogen chloride to the double bond of 2,3-dihydro-4H-pyran (1), followed by replacement of chloride by cyanide on refluxing with silver cyanide in ether ${ }^{102}$; lithium aluminum hydride reduction of the cyano-derivative gave 2-aminomethyl-tetrahydropyran. A similar preparation of the cyano-derivative from 2-bromo-tetrahydropyran has been effected by the action of silver or mercuric cyanide 103 .

When 3,4-d1-0-acetyl-D-xylal (22) in cold benzene solution was saturated with dry hydrogen chloride a syrupy product was obtained on removal of solvent which exhibited no absorption at $1640 \mathrm{~cm}^{-1}$, this indicating that quantitative addition of $\mathrm{HCl}$ had occurred across the double bond of the glycal. Reaction of the product with mercuric cyanide in nitromethane 98 gave a dark coloured product which could not be purifled by chromatography. Although no absorption was present in the $2000-2300 \mathrm{~cm}^{-1}$ region of the infrared spectrum ${ }^{104}$, analysis showed the presence of $2.7 \%$ nitrogen In the chromatographed product. Refluxing the HCl-addition product with silver cyanide in ether ${ }^{102}$ apparently resulted In no reaction other than partial regeneration of (22).

Though these experiments were unsuccessful, they demonstrated the fact that hydrogen chloride adds readily across the double bond of 3,4-d1-0-acetyl-D-xylal (22), with formation of 3,4-di-0-acety1-2-deoxy-D-xylopyranosyl chloride (67). Davoli $1^{105}$ has reported the similar addition of $\mathrm{HCl}$ 


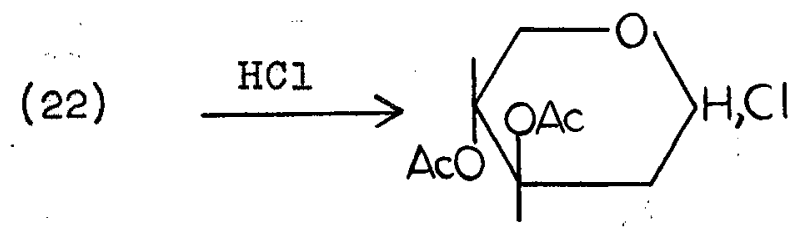

$\underline{46}$

(67)

(and $\mathrm{HBr}$ ) to $3,4-d i-0-a c e t y 1-D-a r a b i n a 1$ (32); the product was not stable, and was not characterised. It was observed that the addition product of hydrogen chloride to (22) also tended to decompose on standing at room temperature (in chloroform solution), and absorption at $1640 \mathrm{~cm}^{-1}$ reappeared in the infrared spectrum. In this respect the glycals resemble 2,3-dihydro-4H-pyran (1) which quantitatively adds hydrogen chloride and hydrogen bromide, but the readily dehydrohalogenated products have not been isolated, and are

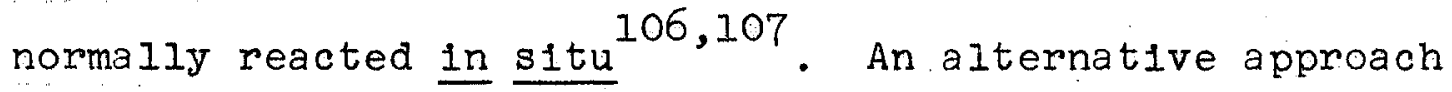
to the problem in hand was based on this observed addition: a methyl, rather than a hydroxymethyl group, was introduced at $C-1$ of the six-membered ring of 3,4-di-D-acetyl-D-xyla 1 (22), and the products thereby obtained were identified With those resulting from reduction of the terminal hydroxymethyl groups of the two 1,5-anhydro-4-deoxy-hexitols, Fractions I and II.

The action of aliphatic or aromatic Grignard reagents on specific functional groups of carbohydrates has been developed by Bonner and co-workers 108 as a versatile tool 
for the introduction of alkyl or aryl groups into various positions of the molecule. In general, two types of reaction are observed;

1. Normal Grignard addition involving carbonyl functions, such as lactones, esters and aldehydes,

2. Metathetical reactions.

of the second type, reactions of particular interest are those of Grignard reagents with polyacetyl-glycosyl halides. Structurally these compounds are hemiacetal halides and therefore resemble $\propto$-chloro-ethers, which have long been known to react with Grignard reagents in the following manner ${ }^{109}$.<smiles>[R]OC([R])[R]([R])CC</smiles>

47

Although the reactions of polyacetyl-glucosyl halldes with Grignard reagents has been a subject of interest since $1906^{110,111,112}$, it was not unt11 1945 that Hurd and Bonner 113 demonstrated metathesis involving the hemlacetal halide, according to equation 47 . The observations of previous workers of addition product formation ${ }^{111}$, or reaction only of the acetyl groups with Grignard reagent ${ }^{110}$ was accounted for by the fact that an insufficient amount of the reagent was used, or that the reaction had been attempted at too low a temperature. 
Bonner 114 showed that preferential addition of Grignard reagent occurs at the ester groups before the metathetical reaction takes place to any appreciable extent; as each ester function takes up 2 moles of Grignard reagent (equation 48), complete reaction of a tetra-0-acetyl-glycosyl halide (equation 49) therefore requires the presence of nine moles
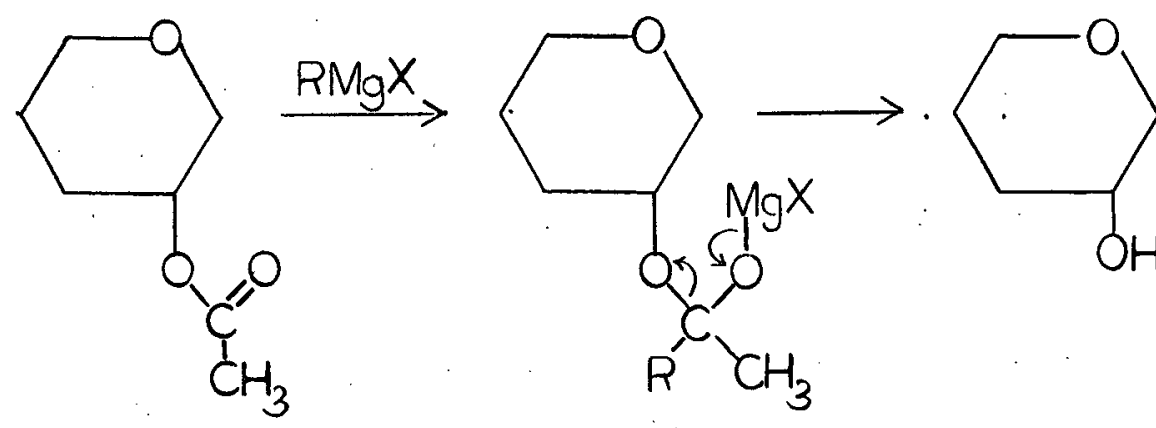<smiles>[R]C([R])(C)O</smiles>

of the reagent. Thus when 2,3,4,6-tetra-O-acetyl- $\alpha-D-$ glucopyranosyl chloride (68) was refluxed in ether with 12 moles of phenyl magnesium bromide, crystalline $(2,3,4,6$ tetra-o-acetyI- $\beta$-D-glucopyranosyl)-benzene (69), accompanied by the syrupy $\propto$-anomer, was isolated after reacetylation $^{113}$.

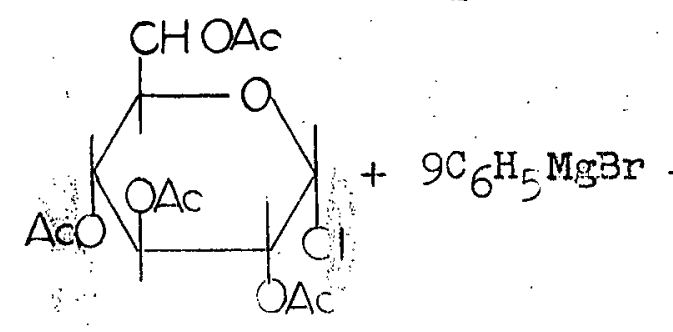

(68)

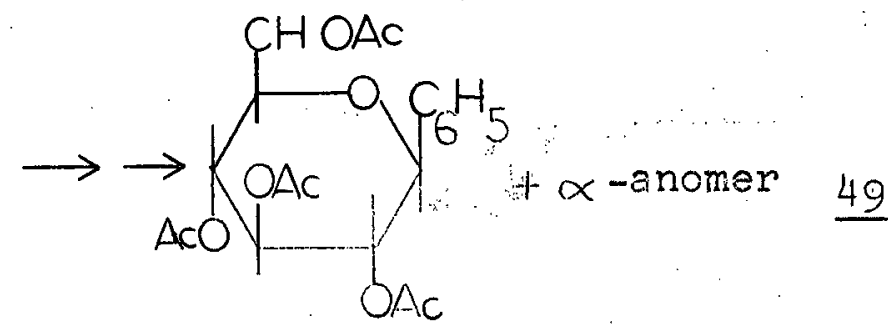

(69) 
Retention of configuration during the formation and subsequent decomposition of the Grignard adduct to the ester function (equation 48) formed the basis of Bonner's invest1gation of this reaction, which was undertaken to determine whether the formation of the same type of product by the aluminium chloride catalysed glycosylation of aromatic compounds ${ }^{115}$ was accompanied by intramolecular isomerisations or inversions. That configuration is retained on deacetylation by Grignard reagents is also a fundamental requirement of our experiment. This point is proved experimentally by the fact that D-glucose is obtalned when $1,2,3,4,6$ penta-O-acetyl- $\beta-$ D-glucose is reacted with Grignard reagents.

The metathetical reaction of Grignard reagents with the acetylated halides of D-glucose, D-xylose and lactose was found by Hurd and Bonner 113 to be an excellent general procedure for the preparation of aldopyranosyl derivatives of aromatic (benzene, toluene, naphthalene) and aliphatic (butane, isopropane) hydrocarbons. By the same procedure, Yoshimura and co-workers have prepared the analogous phenyl, benzyl, methyl, ethyl, propyl and butyl derivatives of 2amino-2-deoxy-D-glucose ${ }^{116}$. The reaction, described below, of a Grignard réagent with a 2-deoxy-glycosyl halide, derived from a glycal, is a hitherto unexplored aspect of this general synthesis. 
Hydrogen chloride was added across the double bond of 3,4-di-0-acetyl-D-xylal (22) (equation 46), and an ethereal solution of the syrupy product (67) was added to a previously prepared solution of methyl magnesium bromide containing a two-fold excess of the Grignard reagent (approximately 10 moles). Reaction and decomposition of the reaction mixture was carried out according to the procedure described by 108

Bonner, except that the product was isolated at this stage in the deacetylated form, as it was hoped to effect a separation by paper chromatography. However, chromatograms of the syrup which was isolated, on developing with a varety of different sovent systems, revealed only one compact zone on spraying with sodium periodate - Schiff reagent ${ }^{73}$. Separation of this component from any impurities not revealed by the spray reagent was effected by preparative paper chromatography, and the purffied material was then subjected to n.m.r. analysis. The spectrum obtalned in deuterium oxide solution clearly showed that the apparently homogeneous product was a mixtuse of two compounds, both containing a $\mathrm{C}-\mathrm{CH}_{3}$ grouping, (and therefore indicated that the attempted reaction (equation 50) had succeeded).

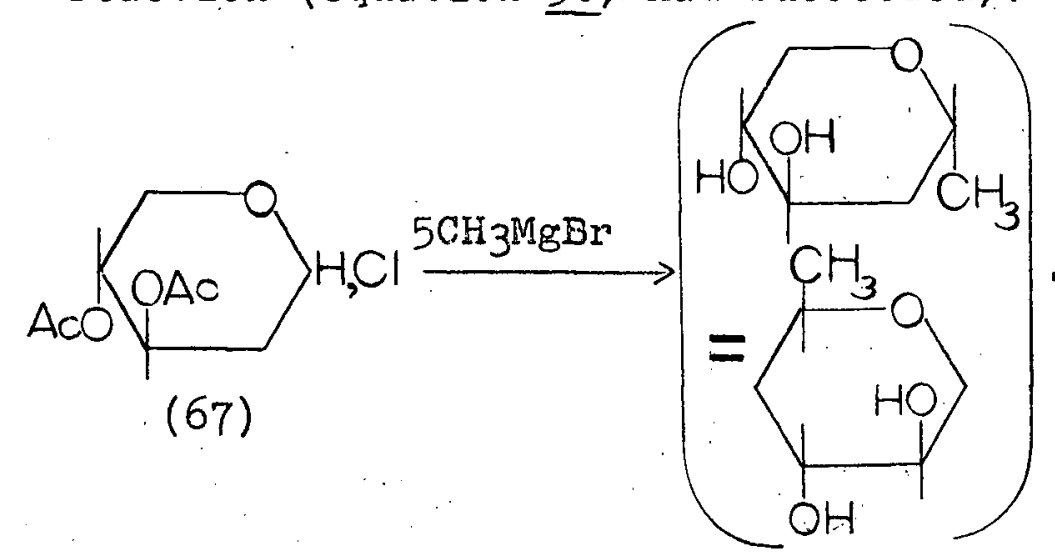

(70)

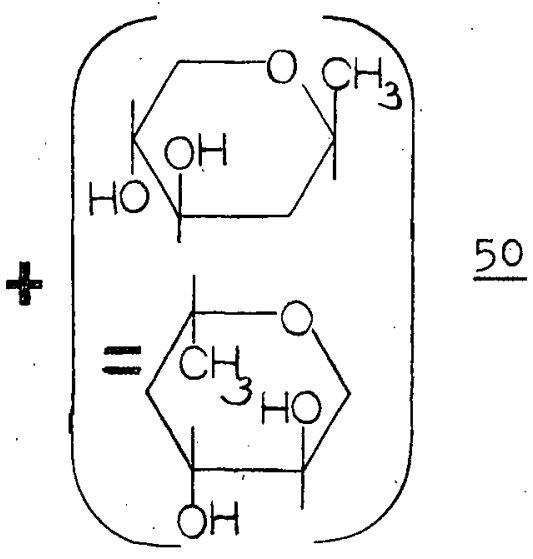

(71) 
This was apparent from the presence of a pair of overlapping doublets $(\mathrm{J}=6 \mathrm{c} / \mathrm{s})$ at high field, $\delta=1.14$ and $1.17 \mathrm{ppm}$. In addition to this absorption in the $\mathrm{C}$-methyl region of the spectrum, a multiplet around $\delta=1.8 \mathrm{ppm}$ was assignable to $\mathrm{C}-\mathrm{CH}_{\mathrm{c}}-\mathrm{C}$, and a complex multiplet between 3.0 and $4.2 \mathrm{ppm}$ to the remaining hydrogens of the rings; the entire spectrum was thus indicative of a mixture of the two anomeric forms of (2-deoxy-D-xylopyranosyl)-methane. Retaining the previous nomenclature, these were 1,5-anhydro-4,6-dideoxy-D-arabinohexitol (70), and 1,5-anhydro-4,6-dideoxy-L-xylo-hexitol (71). The relative intensities of the two methyl doublets showed that the two components were present in a ratio of about $3: 2$.

Resolution of this mixture presented a problem which was solved by gas-liquid partition chromatography (GLPC) of the acetylated anhydrodideoxyhexitols. Acetylation of a portion of the mixture with acetic aninydride-pyridine gave a syrupy product which on thin layer chromatography showed the presence of two barely-resolved components; this was further verification that the initial product isolated from the Grignard reaction was a mixture despite its apparent homogeniety on paper chronatography. After preliminary experiments to establish suitable conditions, it was possible to resolve the mixture of anhydrodideoxyhexitol acetates into two distinct zones by GLPC, using a column (10, $x^{1 / 4}$ ") 
of $20 \%$ Silcone GE-SF-96 on flrebrick at $180^{\circ}$, if the amount of mixture applied to the column in solution was sufficiently smal1. Resolution was less satisfactory when a preparative separation was attempted (approximately $5 \mathrm{mg}$ per injection), and it was necessary to collect fractions enriched in each component and rechromatograph these in order to obtain each component in a pure state, uncontaminated by the other isomer. Each pure component gave an elemental analysis in agreement with an empirical formula of $\mathrm{C}_{10} \mathrm{H}_{16} \mathrm{O}_{5}$. The fastermoving the two components (relative retention time 1.00), $[\alpha]_{D}-21^{\circ}$, was more dextrorotatory than the slower component (relative retention time 1.10), which had a specific rotation of $-82^{\circ}$. Groups of signals present in the n.m.r. spectrum of each fraction, measured in carbon tetrachloride solution, were readily assigned from their resonance positions and relative intensities (in parentheses) to $\mathrm{C}_{-} \mathrm{CH}_{3}$ (3), $\mathrm{C}_{-} \mathrm{CH}_{2}-\mathrm{C}$ (2), acetyl (6) and ring hydrogens with an $\propto$-oxygen (5), and confirmed the identities of the two components as isomeric 2,3-di-0-acetyl-1,5-anhydro-4,6-didecxy-hexitols. On the basis that no inversion of configuration was possible as a result of the reaction of (67) with the Grignard reagent, one of the separated isomers had the D-arabino- (72), and the other the L-xylo- (73) configuration. 


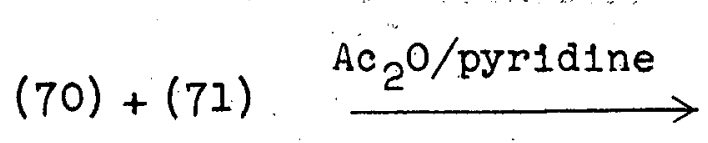

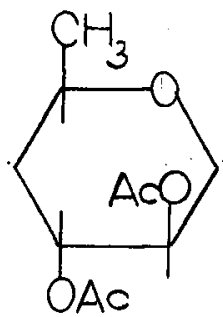

(72)

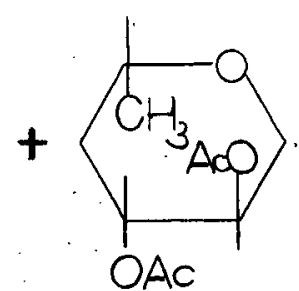

(73)

These same two anhydrodideoxyhexitol diacetates were then synthesised by an alternative route from the mixture of two isomeric 2,3-d1-0-acetyl-1,5-anhydro-4-deoxy-hexitols (74) and (75), which formed the major part of the reaction product from the hydroxymethylation of 3,4-d1-0-acetyl-Dxylal (22), and from which Fractions I and II were obtained on deacetylation. This synthesis involved only the free primary hydroxyl groups at $\mathrm{C}-6$, which were reduced to methyl groups by a series of reactions which have been applied previously to the preparation of 6-deoxy-sugars, such as 6-deoxy-D-glucose $\mathrm{D}^{117}$ and 6-deoxy-D-galactose ${ }^{118}$, from the parent aldoses.

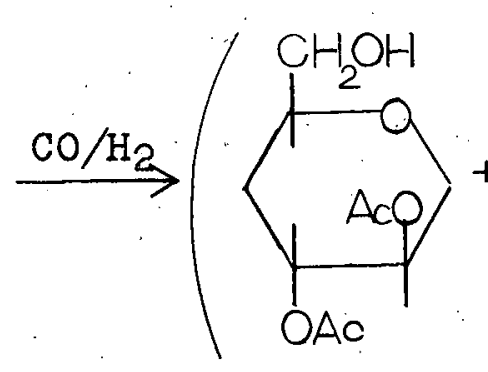

(74)

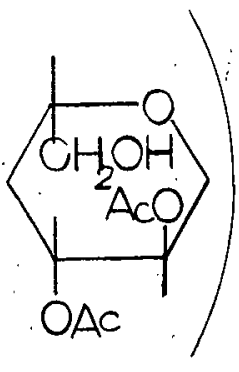

(75)

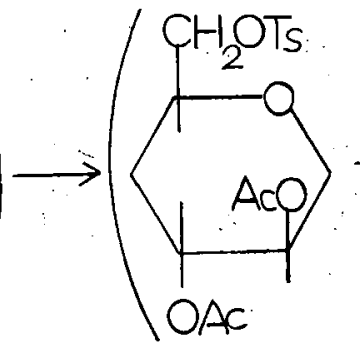

(76)

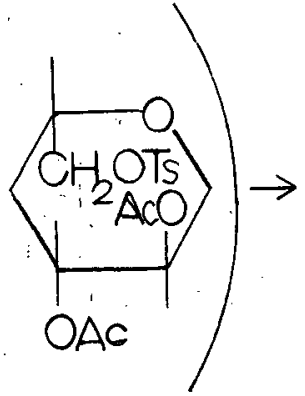

(77) 


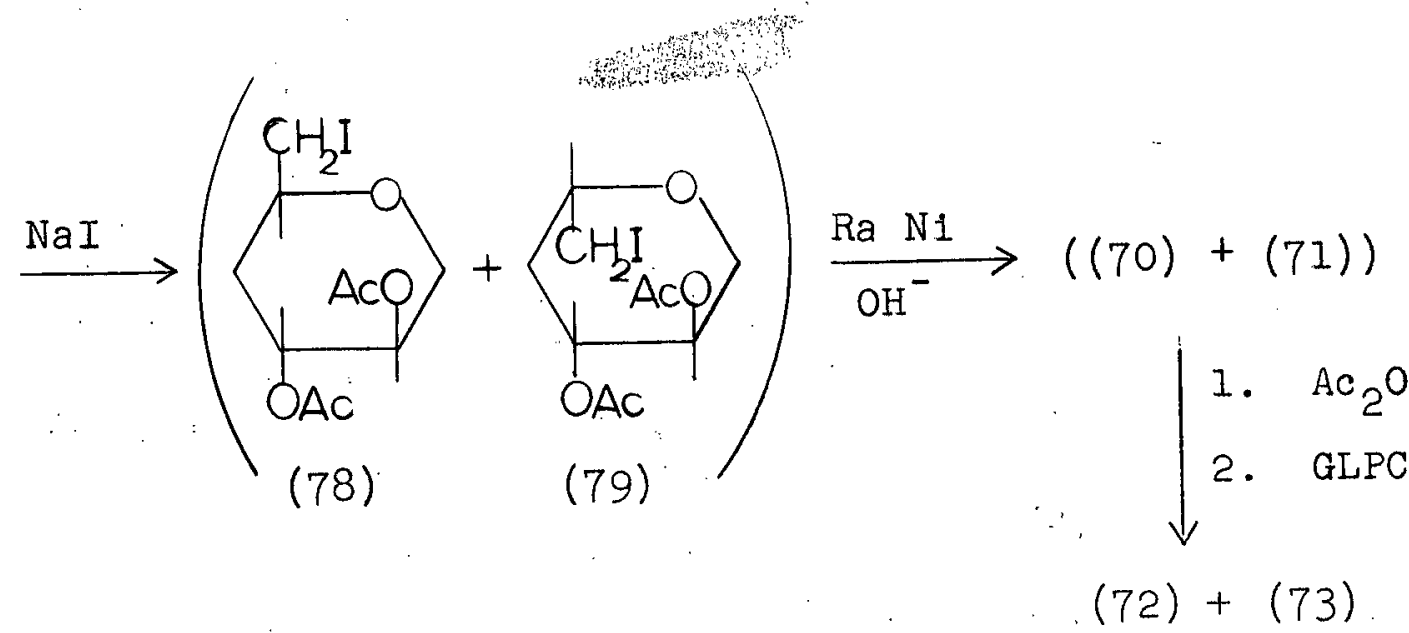

A portion of the syrupy mixture resulting from the oxo reaction of 3,4-di-0-acetyl-D-xylal (22) was treated with an excess of p-toleunesulphonyl chloride in pyridine under standard conditions, and on working up the reaction mixture a product was obtained which, from an examination of the intensities of the characteristic resonances in the n.m.r. spectrum associated with the p-tolylsulphonyl group, relative to those due to absorption by the acetyl groups, contained approximately $75 \%$ of the isomeric 6-0-ptolysulphonyl derivatives (76) and (77). The crude product, in acetone solution, was then heated in a sealed tube in the presence of sodium lodide, whereby the tosyloxy groups were replaced by lodide to give the corresponding mixture of 6-deoxy-6-iodo-derivatives (78) and (79), and sodium p-toluenesulphonate was precipitated. On cooling and filtration, the amount of sodium p-toluenesulphonate isolated 94 agreed closely with the original estimation, from n.m.r. 
data, of the content of isomeric 6-0-p-tolylsulphony derivatives (76) and (77) in the crude mixture. Isolation of the reaction products (78) and (79) from residual sodium lodide was achieved by evaporation of the flitrate to dryness and extraction with ether. When the resulting product, in slightly basic methanol solution, was hydrogenated at atmospheric pressure and temperature in the presence of Raney nickel, according to the procedure of Freudenberg and Raschig ${ }^{118}$, hydrogen was rapidly absorbed and the mixture was simultaneously deacetylated. From this reaction a syrup was 1solated which showed only one zone by paper chromatography, 1dentical in $R_{F}$ value to that of the product obtained from the reaction of 3,4-d1-0-acetyl-2-deoxy-Dxylopyranosyl chloride (67) with methyl magnesium bromide, and which, after purification by paper chromatography, had a n.m.r. spectrum which was essentially identical to that previously obtained. That this was a mixture of the same two D-arabino- (70) and L-xylo- (71) forms of 1,5-anhydro4,6-dideoxyhexitol was demonstrated by acetylation of the purified product and fractionation by GLPC into two components, $[\alpha]_{D}-23^{\circ}$ and $[\alpha]_{D}-81^{\circ}$, which had n.m.r. spectra Identical with those of the two isomeric 2,3-d1-0-acetyl-1,5anhydro-4,6-dideoxy-hexitols (72) and (73), previously described. 
Thus, the fact that the same two compounds were obtained by either route (equations $\underline{50}$ and $\underline{52}$ ) conclusively established that the secondary hydroxyl groups of Fractions I and II had retained the D-threo-configuration present in 3,4-d1-0-acetyl-D-xylal. It was then possible to state with certainty that Fraction I was 1,5-anhydro-4-deoxy-Darabino-hexitol (44), and that Fraction II was 1,5-anhydro-4deoxy-L-xylo-hexitol (45):

Though not an essential part of this proof, 1t was of interest to identify individually the two isomeric diacetates (72) and (73) which were separated by GLPC. It is known that primary hydroxyl groups react with p-toluenesulphonyl chloride at a much faster rate than do secondary hydroxyl groups ${ }^{119}$; it is therefore possible to preferentially sulphonylate a reactive primary position even when other vacant hydroxyls are present. The procedure of unimolar sulphonylation, employing an amount of the sulphonyl chloride in slight excess of that required for esterification of the one reactive site, was first introduced by ohle and Dickhäuser ${ }^{120}$, and later Improved by Levene and Raymond 93 . Generally the reaction rate is lowered by cooling, thereby further reducing the possibility of acylation of the secondary hydroxyls.

$$
\text { 1,5-Anhydro-4-deoxy-L-xylo-hexitol (45), (Fraction II }
$$
from the chromatographic separation of the deacetylated 
hydroxymethylation product from 3,4-d1-0-acetyl-D-xylal) was subjected to unimolar tosylation ( 1.1 molar equivalents of p-toluenesulphonyl chioride), with cooling in ice; after 18 hours acetic anhydride was then added in order to acetylate the secondary hydroxyl groups and facilitate isolation of the product. The resulting syrup, which showed absorption in the infrared spectrum characteristic of both acetyl. and p-tolylsulphonyl groups ( $\mathrm{S}=0$ stretching and aromatic $\mathrm{C}=\mathrm{C}$ stretching vibrations), but not of hydroxyl, was not further purified. It contained approximately $80 \%$ of 2,3-d1-0-acety11,5-anhydro-4-deoxy-6-0-(p-tolylsulphonyl)-L-xylo-hexitol (77), as judged by the amount of sodium p-toluenesuiphonate which was formed when the crude derivative was subsequently heated In a sealed tube with sodium iodide in acetone, during the conversion of (77) to the 6-deoxy-6-iodo-derivative (79). Reductive dehalogenation of (79) in the presence of Raney nickel ${ }^{121}$, and simultaneous deacetylation, then gave 1,5anhydro-4,6-dideoxy-L-xylo-hexitol (73). Following reacetylation, this was readily identified by GLPC with the fastermoving of the two components present in the mixture of anhydrodideoxyhexitol diacetates obtained by the two alternative routes described previously. Thus the more dextrorotatory of the two $\left([\alpha]_{D}-21^{\circ}\right)$ was 2,3-di-0-acetyl-1,5-anhydro-4,6dideoxy-L-xylo-hexitol (73), and the isomer having $[\alpha]_{D}-82^{\circ}$ was the corresponding D-arabino- isomer (72). 
(v) Identities of Polyols $\mathrm{X}$ and $\mathrm{Y}^{82}$

From the results discussed in the previous pages, it was clear that Gorin's assignment ${ }^{82}$ of the D-arabino- configuration to the dextrorotatory 1,5-anhydro-4-deoxyhexitol, (referred to previously as Polyol Y), isolated from the products of hydrogenolysis of methyl $\propto$-D-glucopyranoside $(51)^{80}$, was incorrect. From a consideration of the avaliable evidence, it was possible to deduce the identity of this compound. It was known to be of the D-series, and Gorin's study of the relative rates of lead tetracetate consumption Indicated a trans arrangement of the $\propto-$ glycol group of $Y$. Consequently, it was considered that polyol $Y$ was, in fact, the alternative trans-isomer, 1,5-anhydro-4-deoxy-D-xylohexitol (62), and therefore the enantiomer of Fraction II, the L-xylo-isomer (45).

Through the kind cooperation of Dr. Gorin in supplying a sample of the tris-p-nitrobenzoyl derivative of his polyol, it was possible to prove this point. Debenzoylation of the derlvative with refluxing methanolic sodlum methoxide gave the syrupy polyol, which was found to have a specific rotation of $+40^{\circ}$, equal and opposite to that of the L-isomer (45), and which had a n.m.r. spectrum in deuterium oxide solution 1dentical with that of (45). The syrupy compound formed at crystalline tri-0-acetyl derivative, m.p. 80-82,$[\propto]_{D}+40^{\circ}$, whose infrared spectrum was identical with :that of 2,3,6-tri- 
0-acetyl-1,5-anhydro-4-deoxy-I-xylo-hexitol, m.p. 80-81 ${ }^{\circ}$, $[\infty]_{D}-41^{\circ}$.

Comparison of a sample of the crystalline, levorotatory anhydrodeoxyhexitol. (Polyol $X$ ), also isolated 82 from the hydrogenolysis reaction of (51), with 1,5-anhydro-4deoxy-D-Iyxo-hexitol (60), one of the products obtained from the oxo reaction of 3,4-d1-0-acetyl-D-arabinal $(32)^{122}$, confirmed Gorin's assignment of the structure of this compound. It is noteworthy that the true specific rotation of each polyol ( $\mathrm{X}$ and $\mathrm{Y}$ ) was some $20-30^{\circ}$ lower than the values calculated from an application of the rules of optical superposition (page 62).

(v1) Proton Magnetic Resonance and Stereochemistry of Fractions I and II

It was previously noted (page 44) that the resonance positions and intensities of the high field signals attributed to the $c-4$ hydrogens of (44) and (45), provided evidence for their straight chain anhydrodeoxyhexitol structure, rather than the branched chain structures (46) and (47). In the case of one of the isomers, 1,5-anhydro-4-deoxy-I-xylohexitol (45), additional stereochemical information could be gained from an inspection of the multiplicities of the signals in this region, as well as from the resonance positions of the individual $\mathrm{C}-4$ protons, which was in complete 
agreement with the structure assigned on the basis of the chemical evidence described in the preceeding pages.

Present knowledge of the relation between configurations and conformations of carbohydrates and thelr nuclear magnetic resonance stems from the pioneering work of Lemieux, Kullnig, Bernstein and Schneider ${ }^{123}$. Two factors of importance are the angular dependence of spin-spin coupling constants, and the dependence of chemical shifts on molecular geometry. During their investigations of various acetylated pyranose sugars, Lemieux and co-workers observed that the splitting of the anomeric proton $\mathrm{H}_{1}$, which is readily discernable being at lowest field, was dependent on the relative orientation of $\mathrm{H}_{2}$, with which $1 t$ was coupled. When $\mathrm{H}_{1}$ and $\mathrm{H}_{2}$ were trans-diaxial, the spin-spin coupling constant $\mathrm{J}_{\mathrm{H}_{1}, \mathrm{H}_{2}}$ was two to three times larger than when the neighbouring hydrogens were in other orientations (axial-equatorial or equatorial-equatorial). Thus a large coupling constant was assoclated with a dihedral angle $(\phi)$ of $180^{\circ}$, and a smaller coupling constant with a dihedral angle of $60^{\circ}$. This experimentally observed angular dependence of coupling constants was later generalised by Karplus 124,36 .

In the course of the same investigation on acetylated pyranose sugars, Lemieux and co-workers ${ }^{123}$ observed a difference in resonance positions for chemically identical hydrogens which was related to their orientations in space. Thus 
in $1,2,3,4$-tetra-0-acetyl- $\beta-$ - $x y$ lose $(80)$, the equatorial hydrogen at $\mathrm{C}-5$ was at lower fleld than the $\mathrm{C}-5$ axial hydrogen; similarly, equatorlal anomeric hydrogens invariably resonated at lower magnetic fleld than their axial counterparts. The portion of the spectrum of (80) due to the methy-

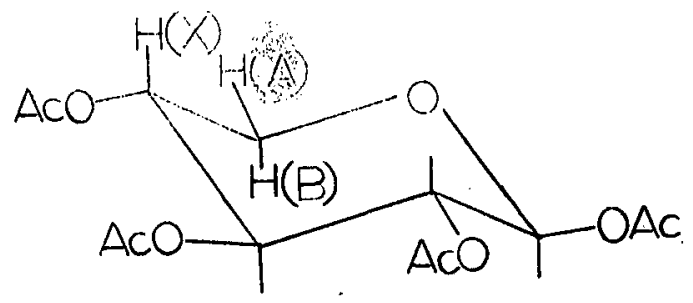

(80)

lene hydrogens at the $C-5$ position was of particular interest, as it was possible to derive parameters for the chemical shifts and spin coupling constants for the individual equatorial (A) and axial (B) hydrogens attached to the same carbon atom, when treated as an ABX system ${ }^{125}$. Values obtained were: $J_{A B}=12 \mathrm{c} / \mathrm{s} ; J_{\mathrm{BX}}=8 \mathrm{c} / \mathrm{s} ; J_{A X}=3.2 \mathrm{c} / \mathrm{s}$. More recently, Woo, Dion and Johnson 126 have made use of the relationships established by Lemieux and co-workers 123 in deducing the complete configurations of methyl chalcoside (81) and of chalcose (82), degradation products of the antiblotic chalcomycin. Their assignment of the configurations 


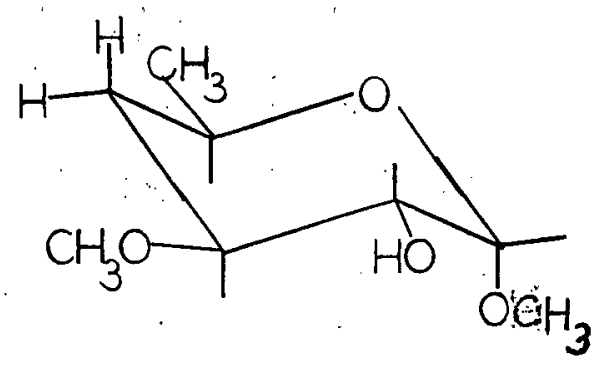

(81)

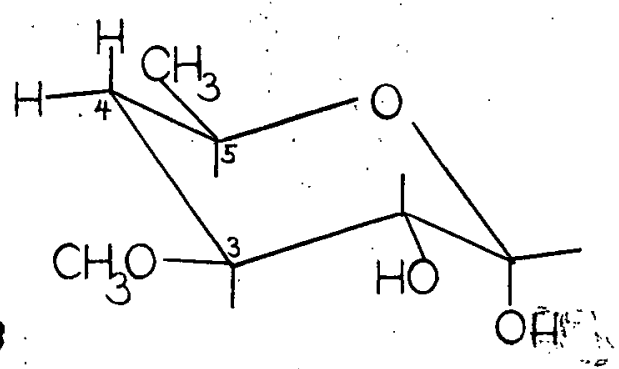

(82)

at $c-3$ and $c-5$ of (81), from the multiplicities of the C-4 proton signals, will be described, as the stereochemistry of the C-3 - C-4 - C-5 fragment of this molecule closely resembles the corresponding portion of 1,5-anhydro-4-deoxyI-xylo-hexitol (45), and therefore provides a model on which to base a discussion of the high field portion of the spectrum of the latter compound. With reference to Figure IA, which shows the signais of the $\mathrm{C}-4$ methylene group of methyl chalcoside measured in pyridine solution; the lower field group around $\delta=2.05 \mathrm{ppm}$ was assigned to the equatorial hydrogen $\left(\mathrm{H}_{4 \mathrm{e}}^{\prime}\right)$, and the broad group of signals at $\delta=1.00$ to 1.58 ppm, partially obscured by the C-methyl doublet, to the axial hydrogen $\left(\mathrm{H}_{4 a}\right)$. Each group of signals was considered as an $A B X$ system $^{125}$ resulting from coupling between $\mathrm{H}_{4 e}, \mathrm{H}_{4 \mathrm{a}}$ and one of the neighbouring protons on $\mathrm{C}-3$ or $\mathrm{C}-5$, the resulting lines then being further split by a fourth hydrogen (on $\mathrm{C}-5$ or $\mathrm{C}-3$ ). On the basis of the observations of Lemieux and co-workers ${ }^{123}$ the spin-spin coupling between the two $\mathrm{C}-4$ 
hydrogens would be expected to be large, of the order of $12 \mathrm{c} / \mathrm{s}$; additional coupling of each proton with neighbouring protons on $\mathrm{C}-3$ and $\mathrm{C}-5$ would also be large if a diaxial relationship existed, otherwise it would be small. The observed splitting of $\mathrm{H}_{4 \mathrm{e}}$ into two quartets indeed showed the anticlpated large coupling $(12.5 \mathrm{c} / \mathrm{s})$ with the geminal $\mathrm{H}_{4 \mathrm{a}}$, but gave no additional information on the relative orientation of $\mathrm{H}_{3}$ and $\mathrm{H}_{5}$. However these could be deduced from the width of the higher field $\mathrm{H}_{4 a}$ signal, $34.5 \mathrm{c} / \mathrm{s}$, this being practically equal to the sum of three coupling constants $\left(\mathrm{J}_{4 a}, 4 \mathrm{e} \mathrm{J}_{4 a, 3}\right.$ and $\left.\mathrm{J}_{4 a, 4 e}\right)$ with which the axial $\mathrm{C}-4$ hydrogen was coupled to the equatorial $\mathrm{C}-4$ hydrogen and to the two. neighbouring hydrogens on $\mathrm{C}-3$ and $\mathrm{C}-5$. Thus; as $\mathrm{J}_{4 a}, 4 \mathrm{e}$ was $12.5 \mathrm{c} / \mathrm{s}, \mathrm{J}_{4 \mathrm{a}, 3}+\mathrm{J}_{4 \mathrm{a}, 5}$ must be $22 \mathrm{c} / \mathrm{s}$; this large value could only be rationalised if $\mathrm{H}_{3}$ and $\mathrm{H}_{5}$ were both axial. Values of $11 \mathrm{c} / \mathrm{s}$ for both $\mathrm{J}_{4 a}, 5 a$ and $\mathrm{J}_{4 a}, 3 a$ satisfactorily accounted for the observed splitting pattern of $\mathrm{H}_{4 \mathrm{a}}$; the two equatorial-axial interactions of $\mathrm{H}_{4 \mathrm{e}}$ were assigned $\mathrm{J}$ values of 2.1 and $5.0 \mathrm{c} / \mathrm{s}$ to account for the observed multiplicity of the lower field group.

The C-4 portion of the n.m.r. spectrum of 1,5-anhydro4-deoxy-L-xylo-hexitol. (45), measured in deuterium oxide solution (Figure 1 B) is seen to bear a close resemblance to the corresponding portion of the spectrum of methyl chalcoside (81); the main point of difference is that the chemical 


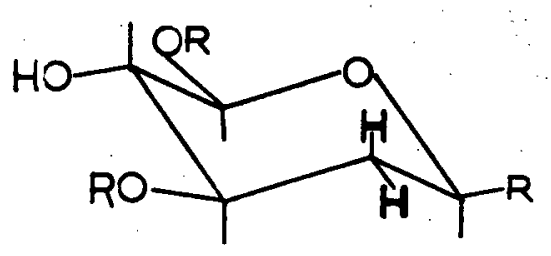

A

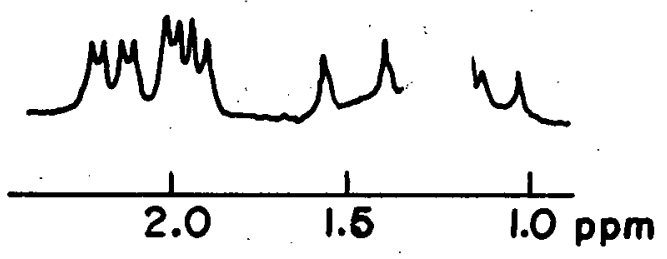

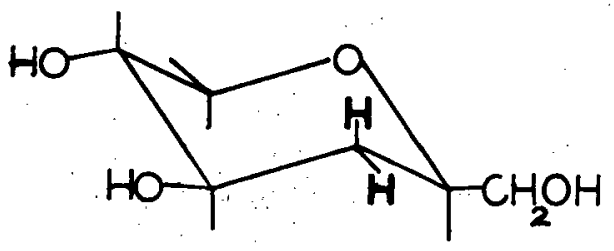

B

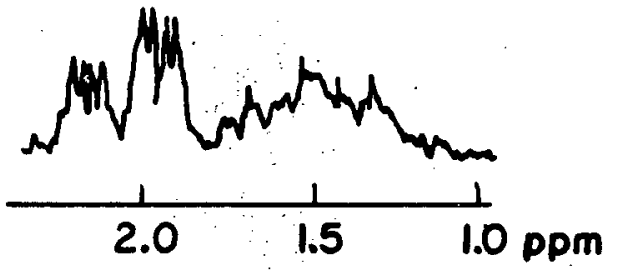

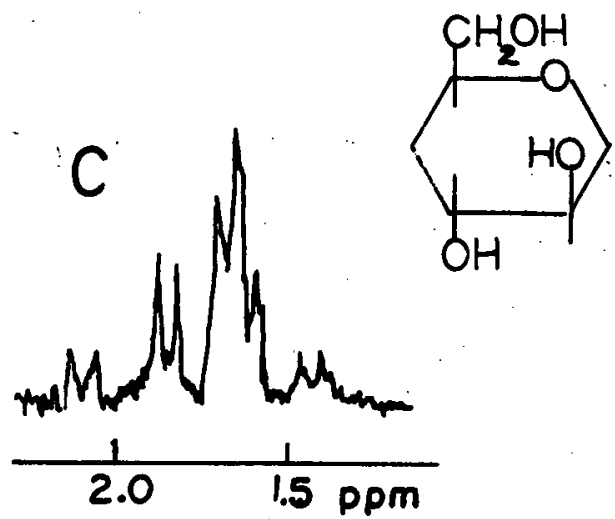

Nmr Spectro (C-4 protons) of : A'Methyl chalcoside

: B Fraction II

:C Fraction I

Figure I 
shift between axial and equatorial. hydrogens at c-4 of (45) is less than was the case with $\mathrm{H}_{4 a}$ and $\mathrm{H}_{4 e}$ of (81), consequently there is no separation between the lower field and higher fleld groups of signals. The splitting of the equatorial hydrogen signal around $\delta=2.0 \mathrm{ppm}$ clearly shows the large spin-spin coupling to the geminal hydrogen ( $\mathrm{J} 4 \mathrm{a}, 4 \mathrm{e}$ approximately $12.5 \mathrm{c} / \mathrm{s})$, and further small splittings by coupling to the adjacent hydrogens on $\mathrm{C}-3$ and $\mathrm{C}-5$ to give a total of 8 lines. The width of the signal of the axial hydrogen on $\mathrm{C}-4$ (about $35 \mathrm{c} / \mathrm{s}$ ) leaves no doubt that $\mathrm{H}_{3}$ and $\mathrm{H}_{5}$ are both axial, as the sum of thelr coupling constants with $\mathrm{H}_{4 a}$ is approximately $22 \mathrm{c} / \mathrm{s}$, as was the case with methyl cralcoside.

The corresponding portion of the spectrum of $1,5-$ anhydro-4-deoxy-D-arabino-hexitol (44) (Figure 1 C) was not amenable to similar analysis; a multiplet was observed between $\delta=1.38$ and 2.15 ppm which could not be separated into axial and equatorial signals.

Deuterated Analogues of (44) and (45)

Several methods are known for simplifying, or otherwise modifying, proton magnetic resonance spectra in order to facilitate their assignments, and a number of these experimental techniques have been discussed by Hall. ${ }^{127}$. As an example of a simple aid to spectral refinement may be 
mentioned the measurement of the spectra of polyols in deuterium oxide, whereby hydroxylic hydrogens are exchanged by deuterlum. A more versatile, though less readily available technique is the replacement of carbohydrate ring hydrogen atoms by deuterium. Although deuterium has a nuclear spin its coupling with adfacent ring hydrogens is so small that their signals are merely broadened and show no resolvable coupling with the deuterium. Consequently, although proton resonance spectra are on the one hand simplified by the substitution of deuterium for hydrogen in the molecule, there 18 at the same time a loss of resolution in the signals of remalning hydrogens which are adjacent to the deuterium atoms. This disadvantage can be overcome by the technique of double resonance, or spin decoupling ${ }^{127}$. Very few examples of the use of deuterated analogues as an ald to the assignment of carbohydrate spectra have as yet been reported $128,129,130$, and double resonance experiments have been confined to the removal of spin coupling between interacting protons 35,131 . The experiments discussed below, whereby specific deuteration was combined with hydrogen-deuterium decoupling, would therefore appear to be the first example of this potentially powerful technique for the simplification of n.m.r. spectra.

By the substitution of deuterium for hydrogen in the previously described oxo reaction of 3,4-di-0-acetyl-D-xylal. 
(22), It was possible to prepare the deuterated analogues ((83) and (84)) of the D-arabino- (44) and L-xylo- (45) 1somers of 1,5-anhydro-4-deoxy-hexitol, in which one of the hydrogens at $\mathrm{C}-4$ and both of the hydrogens attached at C-6. were replaced by deuterium. In order to reduce the amount of deuterium gas required the experiment was performed on a reduced scale, using about $2 \mathrm{~g}$ of the glycal, and the internal volume of the high pressure bomb was reduced to approximately $20 \mathrm{ml}$ by the use of a small glass Iiner, contained in a hollow metal insert which fitted closely inside the bomb. Otherwise, reaction conditions were similar to those described previously. After removal

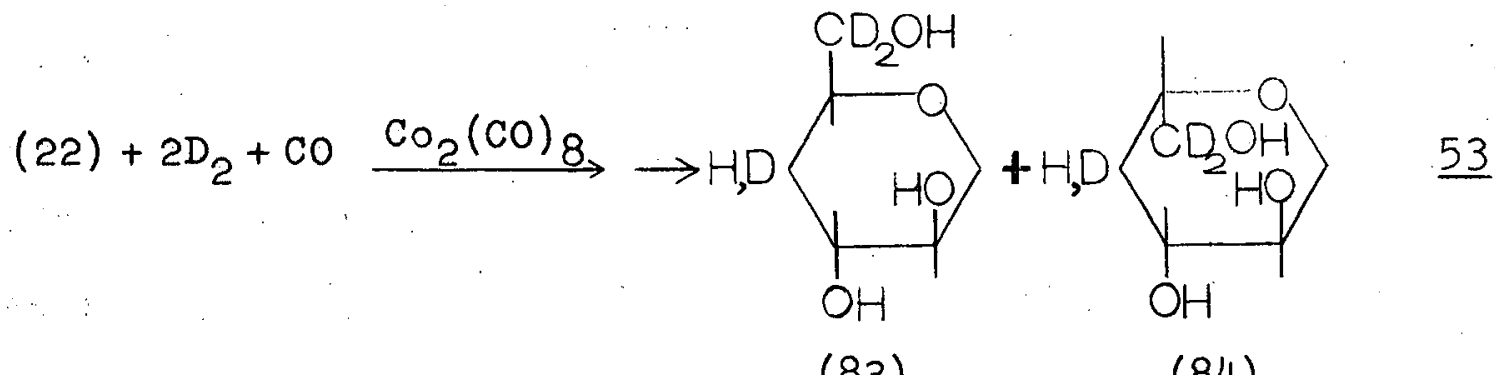

(83)

(84)

of catalyst, and deacetylation with methanolic sodium methoxide, a syrupy product was obtained whose infrared spectrum showed absorption in the region of $2200-2300 \mathrm{~cm}^{-1}$ (C-D stretching). Chromatography on paper revealed two main components with $R_{F}$ values corresponding to those of the normal anhydrodeoxyhexitols (44) and (45); these were separated on a 
preparative scale as described previously. The two chromatographically pure components which were obtained had specific rotations of $-11^{\circ}\left(R_{F}=0.46\right)$ and $-45^{\circ}\left(R_{F}=0.40\right)$. The slower-moving, more levorotatory of the two formed a crystalline acetate with melting point $\left(80-82^{\circ}\right)$ and specific rotation $\left(-42^{\circ}\right)$ very similar to those of the corresponding derlvative of (45). The two fractions were therefore 1,5anhydro-4-deoxy -D-arabino-hexitol-4,6,6- $\mathrm{H}_{3}^{2}$ (83) and 1,5anhydro-4-deoxy-L-xylo-hexitol-4,6,6- $\mathrm{H}_{3}^{2}(84)$.

The n.m.r. spectra of the deuterated 1somers, measured in deuterlum oxide solution at $60 \mathrm{Mc} / \mathrm{s}$, showed in both cases anticipated separation of the ring hydrogen signals into a low field multiplet of relative intensity 6 , and a signal at higher field corresponding to the one hydrogen attached at $\mathrm{C}-4$, thereby providing additional confirmation that the two components separated from the reaction were (83) and (84). The chemical shifts of the C-4 hydrogens (Figure 2) are of particular interest, in that they can be interpreted as providing information on the mode of addition of carbon monoxide and hydrogen to the double bond of 3,4-di-o-acetylD-xylal. It has already been shown, on the basis of the evidence obtained by Lemieux and co-workers ${ }^{123}$, and also by analogy with the fully assigned spectrum of methyl chalcoside ${ }^{126}$, that the equatorial hydrogen at $\mathrm{C}-4$ of 1,5 -anhydro-4-deoxy-Lxylo-hexitol (45) resonates around $\delta=2.0 \mathrm{ppm}$, whereas the 


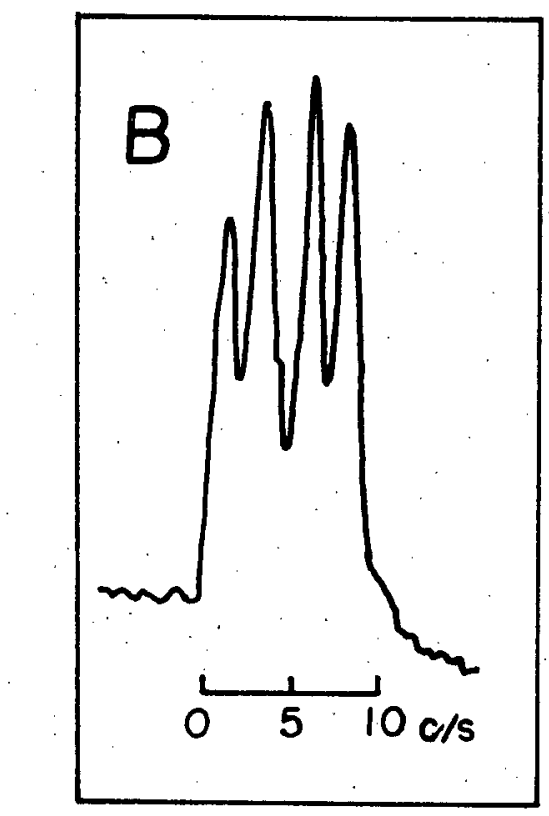

Decoupled

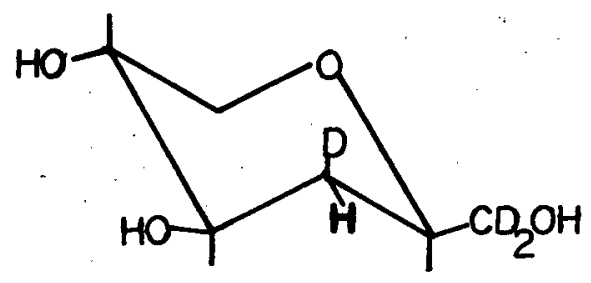

A

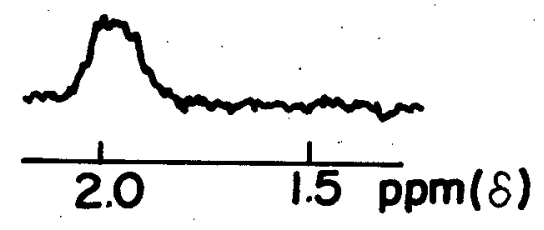

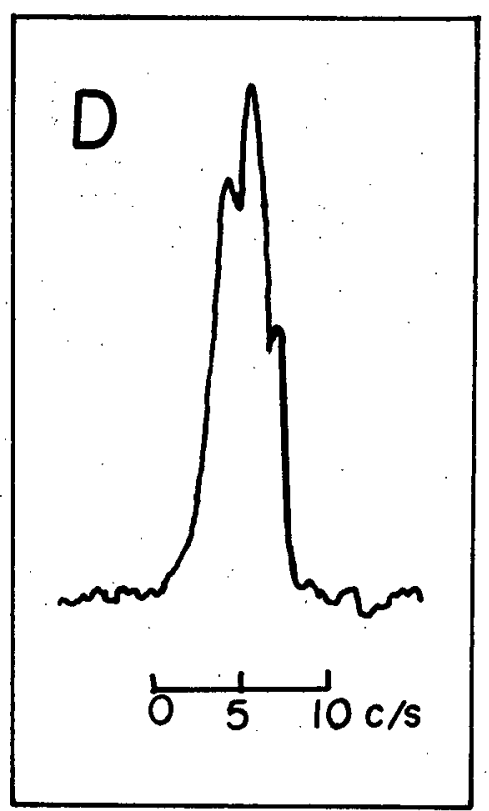

Decoupled

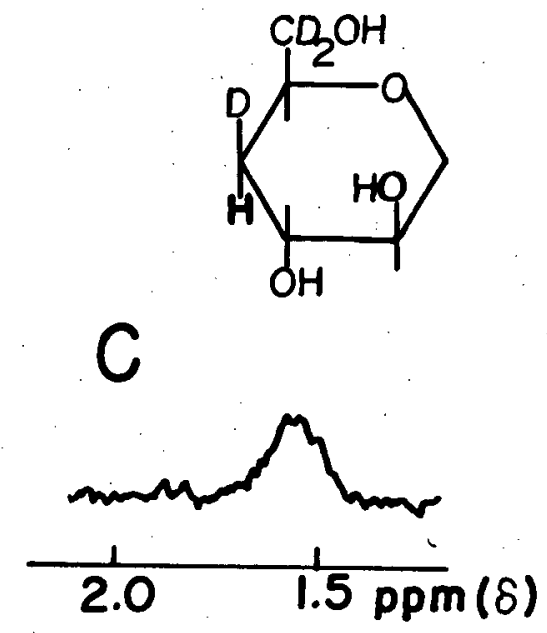

Nmr Spectra(H4) of Deuterated Hexitols

Figure 2 
chemical shift of the axlal hydrogen is about $1.5 \mathrm{ppm}$. Therefore it can be assumed that the single $c-4$ proton signal of (84), the deuterated analogue of (45), at about $\delta=2.0 \mathrm{ppm}$ (Figure 2A) is due to an equatorial hydrogen, and consequently the deuterium atom at $\mathrm{C}-4$ is in an axial orientation (85). This assumption is supported by the fact that the width of

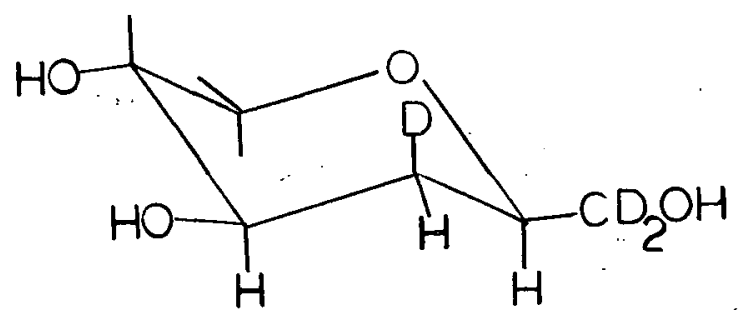

(85)

the signal at $\delta=2.0 \mathrm{ppm}$ is only about $9 \mathrm{c} / \mathrm{s}$; a single axial hydrogen at $\mathrm{C}-4$ would be coupled with the two axial hydrogens at $\mathrm{C}-3$ and $\mathrm{C}-5$, and would therefore have a band width of the order of $20 \mathrm{c} / \mathrm{s}$.

Most convincing evidence for the equatiorial orientation of $\mathrm{H}_{4}$ was provided by the deuterium-decoupled spectrum of (85), also measured in deuterium oxide solution. Whereas in the normal spectrum (Figure $2 \mathrm{~A}$ ), lines resulting from the coupling of $\mathrm{H}_{4}$ with $\mathrm{H}_{3}$ and $\mathrm{H}_{5}$ were broadened by additional coupling with the gem-deuterium atom, and an unresolved 'envelope' was observed, deuterium-hydrogen spin decoupling effectively resolved the signal into.a sharply-defined quartet 
(Figure 2B), resulting from the splitting of $\mathrm{H}_{4}$ (by $\mathrm{H}_{3 a}$ or $\mathrm{H}_{5 \mathrm{a}}$ ) into a doublet, which was further split by the other axial hydrogen. The two coupling constants of 2.3 and $5.1 \mathrm{c} / \mathrm{s}$ which fit this observed splitting pattern could only be accounted for by the fact that $\mathrm{H}_{4}$ was in a gauche relationship to the two axial hydrogens at $\mathrm{C}-3$ and $\mathrm{C}-5$. Hence the deuterium attached at $\mathrm{C}-4$ and the $\mathrm{CD}_{2} \mathrm{OH}$ group attached at $\mathrm{C}-5$ were $\mathrm{cis}$, and the deuterated anhydrodeoxyhexitol (85) must have been formed by a cis addition to the double bond of 3,4-di-0-acetyl-D-xylal. This evidence for cis addition in the oxo reaction of glycals therefore, supports previous experimental evidence obtalned with other unsaturated compounds 29,30 , and is compatible with currentiy acceptable theories of the mechanism of this reaction 17,18 which were discussed in the General Introduction.:

On this evidence it was supposed that the Isomeric deuterated anhydrodeoxyhexitol (83) must also have the deuterium atom at $\mathrm{C}-4$ and the $-\mathrm{CD}_{2} \mathrm{OH}$ group at $\mathrm{C}-5$ in $\mathrm{C}$ is relationship (86): indeed, the chemical shift of the single proton at $\mathrm{C}-4$, $\delta=1.55 \mathrm{ppm}$, could well be assigned to an axial hydrogen as in (87). However the high field portion

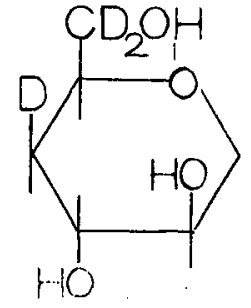

(85) of the spectrum of the normal D-arabinoisomer-(44), (Figure IC), did not permit the assignment of chemical shifts to the individial hydrogens at $\mathrm{C}-4$; furthermore, 
the width of the C-4 signal (Figure 2C) is much less than would be anticipated for an axial hydrogen coupled with an

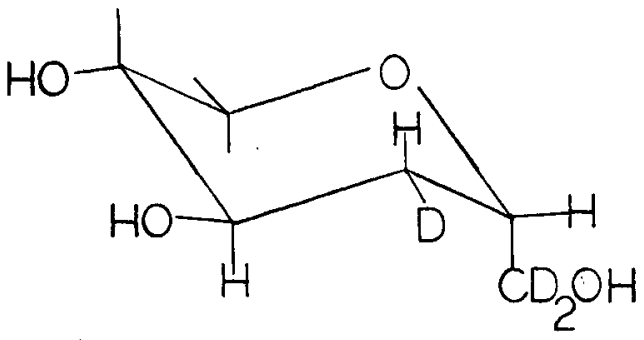

(87)

axial hydrogen at $\mathrm{C}-3$ and an equatorial hydrogen at $\mathrm{C}-5$, as in (87). The splitting pattern of the $\mathrm{C}-4$ hydrogen in (83) which was revealed on hydrogen-deuterlum decoupling (Figure 2D) showed a barely resolved triplet. Thus the coupling constants of $\mathrm{H}_{4}$ with, the two adjacent hydrogens at $\mathrm{C}-3$ and $\mathrm{C}-5$ must be very small, and on the basis of Karplus' parameters ${ }^{127}$ this indicated that the dihedral angles between $\mathrm{H}_{4}$ and $\mathrm{H}_{5}$, and $\mathrm{H}_{4}$ and $\mathrm{H}_{3}$ were both not far removed from $90^{\circ}$. These data were not consistent with a normal chair form (87) for (83).

B. Anhydrodeoxyheptitols from 3,4,6-Tr1-0-acetyl-D-galactal

In 1957 Rosentha: and Read ${ }^{63}$ described the reaction of 3,4,6-tri-0-acetyl-D-galactal (40) with carbon monoxide and hydrogen under oxo conditions. Following deacetylation of the catalyst-free reaction product, one compound, m.p. 158$159^{\circ},[\propto]_{D}+38^{\circ}$, was isolated by cellulose column chromatcgraphy 
which, from its empirical formula and those of its crystalline benzoyl and p-nitrobenzoyl derivatives, gave indication that a hydroxymethyl group had been added to the double bond of the glycal during the course of the oxo reaction. From a study of the oxidation and over-oxidation of this product by periodate 132 , resulting in the total consumption of 6 moles of oxidant and the liberation of 2 moles of formic acid and one of formaldehyde, it was concluded that the hydroxymethyl group had probably added at $\mathrm{C}-2$ of the glycal, giving rise to the branched chain carbohydrate (88) of unknown conflguration at $\mathrm{C}-2$. The work described in this

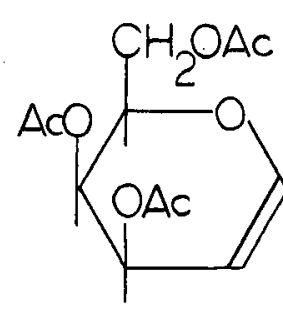

(40)
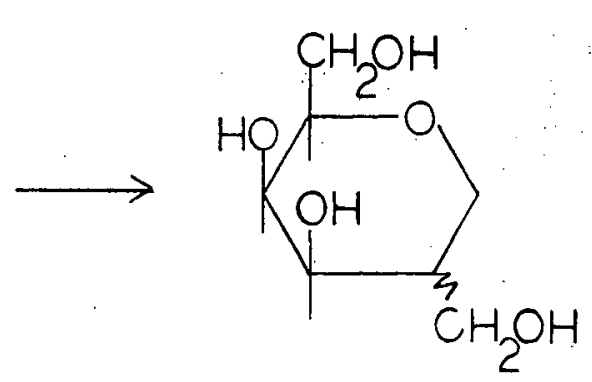

(88)

section comprises a further investigation of the oxo reaction of 3,4,6-tri-o-acetyl-D-galactal.

\section{(i) Reaction Conditions \\ (a) 3,4,6-Tri-0-acetyl-D-Galactal (40)}

The flrst recorded preparation of (40) by Levene and Tipson ${ }^{133}$ used the procedure of Fischer ${ }^{31}$; other modifications: 
have been described by Bates and co-workers ${ }^{134}$, and by Overend and co-workers 42 . In this work the convenient $\cdots$ procedure of Helferich and co-workers 45 was used for the preparation of 3,4,6-tri-o-acetyl-D-galactal, and on one occasion a good overall yield (76\% from D-galactose (18)) of pure glycal was obtained on distillation of the crude reaction product. However, other attempts at purification, following

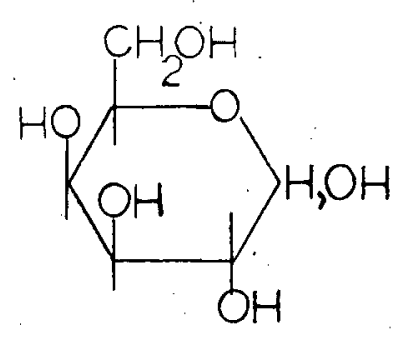

(18)

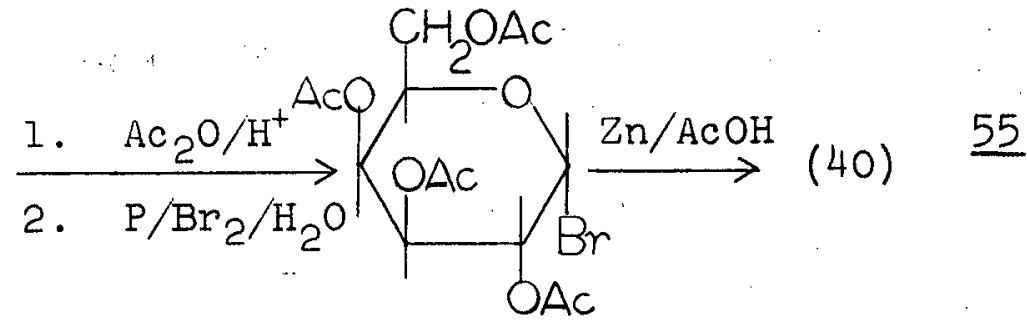

(89)

preparation by the same prociedure, resulted in rapid decomposition with evolution of acetic acid when the crude product, known to contain a high proportion of the glycal, was heated under vacuum. Little improvement was obtained when the procedure of Helferich 45 was modified by preliminary isolation and purification of the intermediate 2,3,4,6-tetraO-acetyl- $\propto$-D-galactosyl bromide (89). In cases where distillation resulted in decomposition, it was found possible to effect a purification of small amounts of the crude product by chromatography on a column of Florisil. 
(b) Reaction Conditions and Product Isolation

Conditions employed for the reaction of 3,4,6-triO-acetyl-D-galactal (40) with 3 moles of synthesis gas $\left(\mathrm{CO}+2 \mathrm{H}_{2}\right)$ were in general similar to those used by Rosenthal and Read ${ }^{63}$, and to those previously described in Section A for the corresponding reaction of 3,4-di-0acetyl-D-xylal (22). Previous work on the oxo reaction of glycals has indicated that the acetylated hexals are somewhat less reactive than the pental derivatives, therefore a slightly higher reaction temperature $\left(135^{\circ}\right)$ was used.

Reaction products were separated from catalyst and isolated as previously described in Section $A$, to give a syrupy product whose infrared spectrum showed that addition to the double bond was complete (absence of $\mathrm{C}=\mathrm{C}$ stretching. vibrations around $1640 \mathrm{~cm}^{-1}$ ), and which showed a hydroxyl band of moderate intensity at $3400 \mathrm{~cm}^{-1}$. The spectrum was therefore compatible with the presence of one or more sugar alcohols. No attempt was made to fractionate the product In the acetylated form, as thin layer chromatography showed a mixture of components which was not well resolved. The product was then deacetylated with methanolic sodium methoxide 72 to give a partially crystalline material which was examined by paper chromatography, using the previously described solvent system of water-saturated l-butanol containing 
$5 \%$ ethano1. When development of the chromatogram was interrupted at the point where the solvent front was near the lower edge, only one major zone of low mobility was apparent on spraying with periodate-Schiff reagent 73 . However on developing the chromatograms for increasingly longer periods the apparently homogeneous main zone became resolved into two distinct components, which after development for about 60 hours at room temperature were near the leading edge of the paper and sufficiently separated to justify an attempted fractionation on a preparative scale. Fractionation of a portion of the deacetylated product was carried out by essentially the same procedure used to separate the anhydrodeoxyhexitols derived from 3,4-di-O-acetyl-D-xylal (Section A). Recovery of the two fractions, which were present in approximately equal amounts, represented about $75 \%$ of the material applied to the chromatograms. For the purposes of subsequent discussion the faster-moving of the two will be referred to as Fraction $A$, and the slower as Fraction $B$.

\section{(i1) Characterisation of Fractions A and B}

$R_{F}$ values of the two fractions were measured on paper with the same solvent system used for their separation, confirming that each was chromatographically pure. Both fractions were obtained in crystalline form, and gave elemental analyses corresponding to the anticipated empirical formula 
$\left(\mathrm{C}_{7} \mathrm{H}_{14} \mathrm{O}_{5}\right)$ required by the addition of a hydroxymethyl group to the glycal double bond.

\begin{tabular}{lrll} 
& m.p. & $R_{F}^{(a)}$ & {$[\alpha]_{D}^{(b)}$} \\
\cline { 2 - 4 } Fraction A & $158-9^{\circ}$ & 0.24 & $24^{\circ}$ \\
Fraction B & $168^{\circ}$ & 0.21 & $68^{\circ}$
\end{tabular}
(a) in water-saturated I-butanol $+5 \%$ ethanol at room temperature

(b) In water at room temperature

Fractions $A$ and $B$ were both characterised as crystalline p-nitrobenzoyl derivatives, prepared by reaction with p-nitrobenzoyl chloride in pyridine 82 , whose analyses were compatible with the esterification of four hydroxyl groups in each case.

\section{(1ii) Structures of Fractions A and B}

It was possible to determine the molecular structures of Fractions $A$ and $B$ by a similar approach to that used in determining the structures of anhydrodeoxyhexitols I and II (Section A). However, the problem of determining the absolute stereochemistry of the two Fractions was not amenable to solution by classical methods.

In view of the earlier investigation carried out by Rosenthal and Read ${ }^{63}$ on the identity of the single polyol, 
1solated from the oxo reaction products of 3,4,6-tri-0-acetylD-galactal, which was thought to have a branched-chain structure (88), it was of particular interest to establish the configurations of Fractions $A$ and $B$ obtained from the same reaction. As with the hexitols (Fractions $I$ and II) derived from the oxo reaction of 3,4-d1-0-acetyl-D-xyla1,-it was again assumed that four isomeric compounds, (90) - (93), were theoreticai possible by the addition of a hydrogen atom and a hydroxymethyl.group at either end of the double bond of (40). Consideration of these four possible strucfures

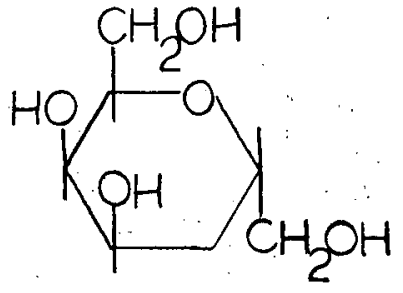

(90)

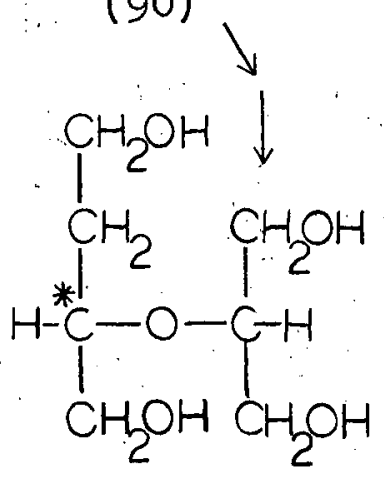

(94)

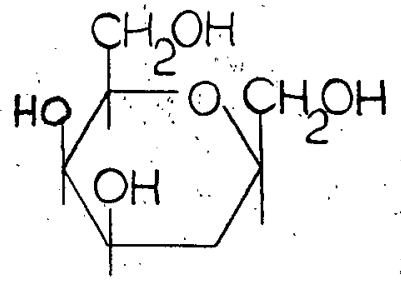

(91)

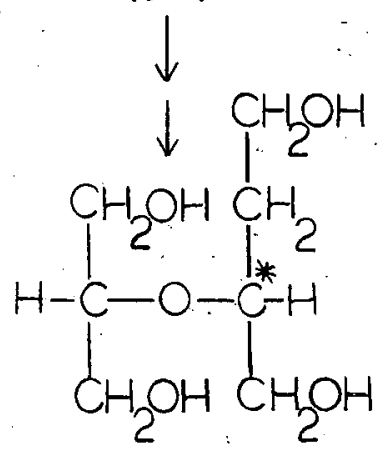

(95)

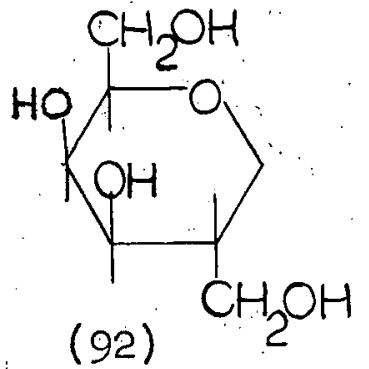

(92)

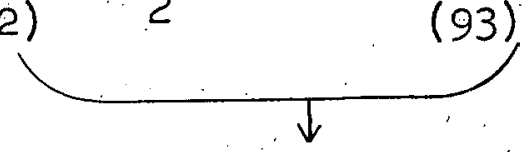

(93)

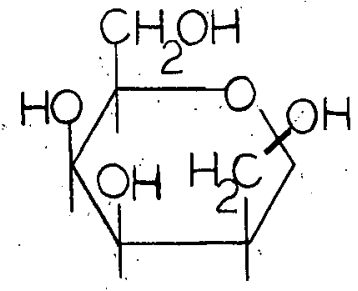<smiles>OCC(CO)CC(CO)CO</smiles>

(96)

shows that the argument previously applied in deciding between branched and straight-chain structures for Fractions I and II was equally valid inthis case. Periodate cleavage 
of the $\alpha-$-glycol group of any one of the four isomeric polyols (90) - (93) and reduction of the dialdehyde so formed would afford a tetrol ether which would be optlcally active, having one asymmetric carbon atom (*) if formed from one of the straight chain heptitols (90) or (91), but would be devoid of asymmetry and therefore optically inactive (96) If derived from elther of the branched isomers (92) and (93).

When Fractions $A$ and $B$ were separately oxidised with an excess of periodic acid, and the resulting dialdehydes, after neutralisation of the reaction medium, were reduced in aqueous solution with sodium borohydride, syrupy products were obtained after working up the reaction in the usual way which had equal and opposite rotations; the tetrol ether from Fraction $A$ was dextrorotatory $\left([\alpha]_{D}+21^{\circ}\right)$, and that from Fraction $B$ was Ievorotatory $\left([\alpha]_{D}-23^{\circ}\right)$. Confirmation of the structures of the tetrol ethers thus obtained was provided by their n.m.r. spectra, measured in deuterium oxide solution. These were identical and showed a multiplet at lower field ( $\delta=3.50-3.85 \mathrm{ppm})$ and a group at higher field $(\delta=1.47-1.02 \mathrm{ppm})$ which had the appearance of a quartet of lines. The relative intensities of the two groups of non-hydroxylic hydrogen signals were in the ratio of $10: 2$, consistent with the 2deoxy-3-0-(1,3-dihydroxy-2-propyl)-glycero-tetritol structure 
of tetrol ethers (94) and (95), but not with the structure of the tetrol ether (96), which would have been formed had either Fractions A or B been branched chain structures, as (96) contains only one hydrogen attached to a carbon atom without an $\alpha$-oxygen function, and eleven other nonhydroxylic hydrogens which would resonate at lower fleld ${ }^{79}$. Apart from the relative intensities of the low and high fleld groups of signals, the close similarity between the spectra of the tetrol ethers obtained from these reactions and those of the enantiomeric 2-deoxy-3-0-(2-hydroxyethyl)glycero-tetritols (48) and (49) previously derived from the straight chain anhydrodeoxyhexitols (44) and (45) is worthy of note. The tetrol ethers from Fractions $A$ and $B$ were characterised as the tetra-0-p-nitrobenzoyl derivatives, prepared and isolated in the usual way. The derivative of Fraction $A, m . p .150-151^{\circ},[\alpha]_{D}+23^{\circ}$, had an infrared spectrum identical with that prepared from Fraction $B$, which had m.p. $150-151^{\circ},[\alpha]_{D}-23^{\circ}$, these data providing further evidence for the enantiomeric nature of the two tetrol ethers.

Apart from the evidence thus obtained from their degradations to optically active compounds, the n.m.r. spectra of the two polyols $A$ and $B$ (Figure 3) were also indicative of the fact that both had straight chain, rather than branched chain structures. Measured in deuterium oxide solution, the spectra of both $A$ and $B$ showed separation of signals into a 

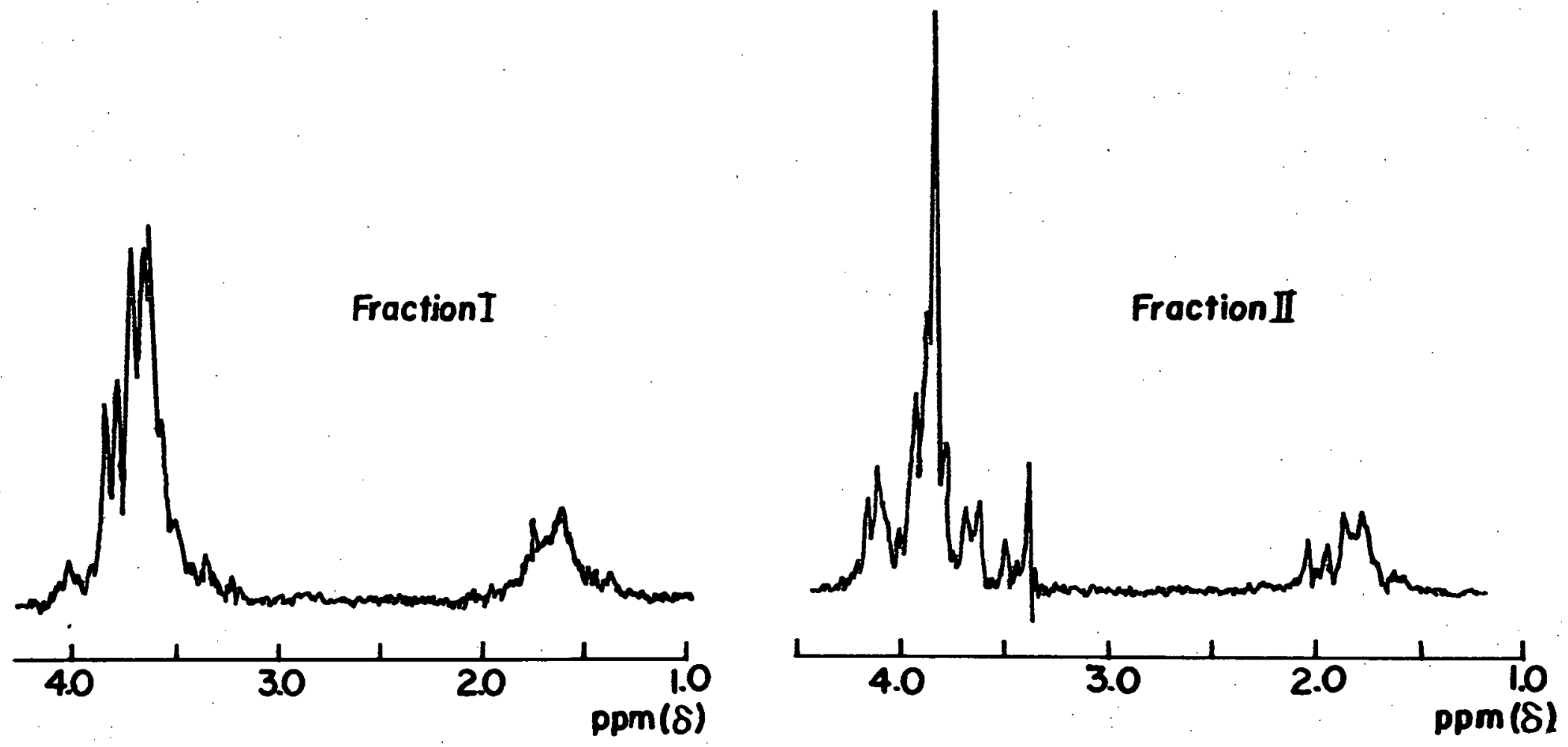

Nmr Spectra of Anhydrodeoxyheptitols

Figure 3 
higher field group within the region of $\delta=1.3-2.1 \mathrm{ppm}$ (area $=2$ ) and a complex mult1plet with a relative area of 8 around $\delta=3.2-4.3 \mathrm{ppm}$. The observed intensities were compatible only with structures (90) and (91), as in $\mathrm{D}_{2} \mathrm{O}$ solution either of the branched chain polyols (92) and (93) would have only a single hydrogen $(\mathrm{C}-\mathrm{CH}-\mathrm{C})$ resonating in the higher field region, and nine other less shielded hydrogens. Ne1ther spectrum (Figure 3) showed any spin-spin multiplicities of value in distinguishing between the two structures (90) and (91).

On this evidence it could be concluded that both Fraction $A$ and Fraction $B$ were 2,6-anhydro-3-deoxy-heptitols, and therefore differed only in configuration at $\mathrm{C}-2$. Thus one of the two fractions was 2,6-anhydro-3-deoxy-D-galactoheptitol (91), forming the tetrol ether 2-deoxy-3-0-(1,3dihydroxy-2-propyl)-I-glycero-tetritol (95) on cleavage with perlodate and reduction with sodium borohydride, and the other fraction was the corresponding D-talo-1somer (90), from which the tetrol ether (94) having the D-configuration was obtained. 


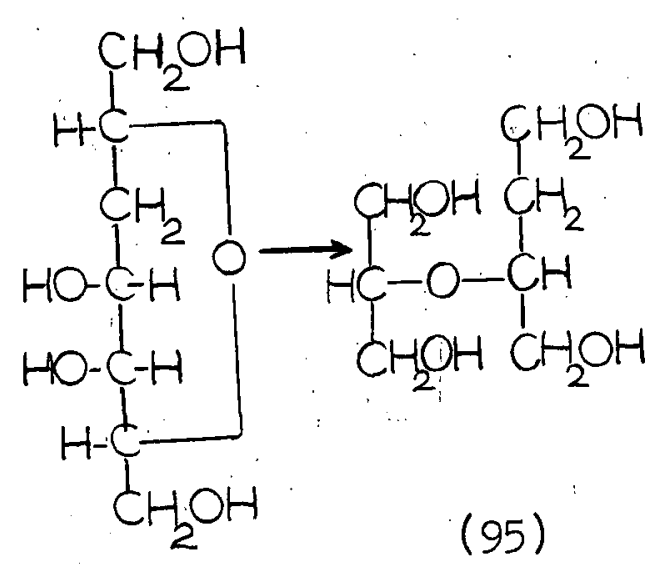

(91)

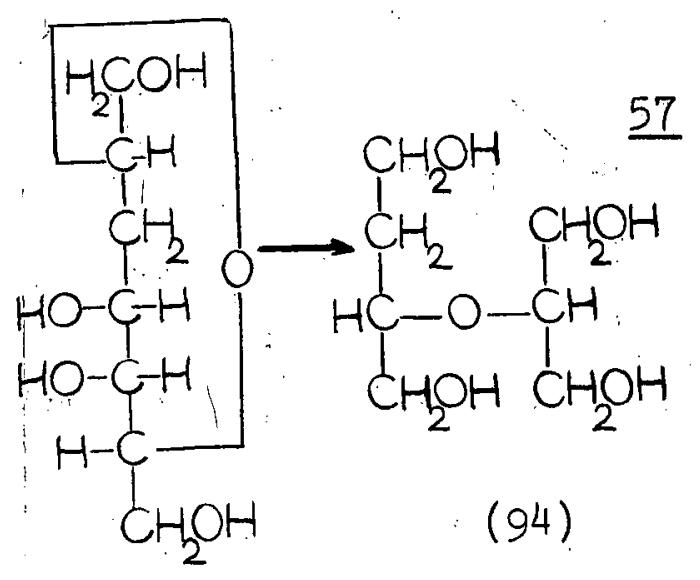

(90)

\section{(iv) Stereochemistry of Fractions A and B}

(a) Periodate Consumption and Acetalation

Determination of the amount of periodate ion consumed by both Fractions $A$ and $B$, by the previously described spectrophotometric method ${ }^{76}$, confirmed the presence in each compound of one $\alpha-$ glycol group, one molar equivalent of periodate ion reacting in each case. The rates of reaction with periodate ion relative to those previously determined for the two anhydrodeoxyhexitols (44) and (45) were of interest, the reaction being much faster for the two anhydrodeoxyheptitols $A$ and $B$ than for (44) and (45), in which a trans arrangement of secondary hydroxyl groups was present. This is clearly shown in the table on the next page, which compares the mole fraction of periodate ion consumed against time for anhydrodeoxyhexitol (45) and anhydrodeoxyheptitol $B$, 
which were reacted under identical conditions.

\begin{tabular}{|l|l|l|l|l|l|l|l|}
\hline Time & 1 min. & $47 \mathrm{~min}$. & $1.5 \mathrm{hrs}$. & $3 \mathrm{hrs}$. & $10.5 \mathrm{hrs}$. & $17 \mathrm{hrs}$ & $24 \mathrm{hrs}$. \\
\hline (a) & 0.63 & 0.97 & & & & 0.87 & \\
(b) & & & 0.40 & 0.59 & 0.95 & & 0.88 \\
\hline
\end{tabular}

Moles of Periodate Ion Consumed per Mole of Substrate for (a) 2,6-Anhydro-3-deoxy-heptitol (Fraction B); (b) 1,5-Anhydro-4-deoxy-E-xylo-hexitol (45).

The exact mechanism by which the carbon-carbon bond connecting the adjacent hydroxyl groups of an $\alpha-g l y c o l$ is broken has not been established conclusively, but a cyclic ester structure such as (97), first proposed by Criegee ${ }^{135}$, is generally considered to be an intermediate in the process $^{74}$. The well known difference in rate of periodate<smiles>CC1(C)OO[13C]([18OH])OC(C)(C)O1</smiles>

(97)

oxidation between cls and trans $\propto-g$ lycol groups attached to a six-membered ring 136 has been rationalised on conformational grounds by Honeyman and Shaw ${ }^{137}$. Variations observed in the rate of perrlodate consumption of a number of pyranoside derivatives, in which only one $\alpha$-glycol group 
of known conformation was ava1lable for oxidation, were consistent with the fact that the rate was dependent on the relative ease of formation of a cyclic intermediate such as (97). Attachment of such a 5-membered cyclic structure onto a pyranoid ring would require the $\mathrm{C}-\mathrm{O}$ bonds of the $\alpha-g l y c o l$ group to be constrained into a greater degree of coplanarity for both cis-(axial-equatorial)(98) and trans(equatorial-equatorial): (99) diols. In the latter case (99), as the two carbon atoms of the diol syste are rotated in order to bring the two attached oxygen atoms closer together, the ring becomes more puckered; consequently the axial

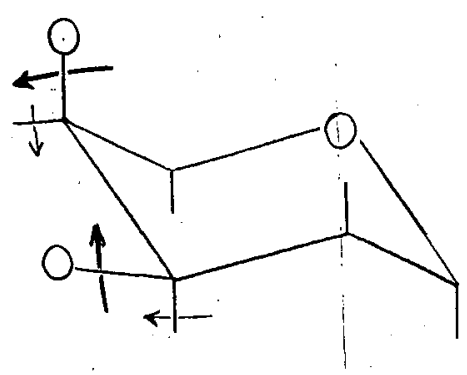

(98)

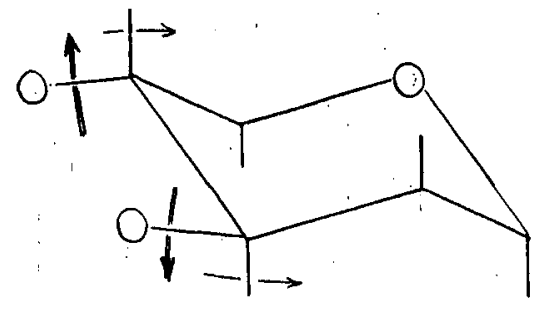

(99)

axial substituents on the ring are brought closer together and considerable energy is required to overcome the resulting repulsions. The reverse applies with cis (axial-equatorial) systems (98), where a similar operation results in the ring becoming less puckered, and interatomic repulsions are not appreciably changed. Formation of a cyclic intermediate therefore proceeds readily. The rapid rates of perlodate oxidation 
of Fractions $A$ and $B$ relative to those of anhydrodeoxyhexitols (44) and (45) thus demonstrated the c1s configuration of the secondary hydroxy 1 groups at $\mathrm{C}-4$ and $\mathrm{C}-5$ of the two anhydrodeoxyheptitols.

A similar explanation for the more ready formation of the O-isopropylidene derivatives of an $\alpha$-glycol group situated on a six-membered ring when the hydroxyl groups are cis, rather than trans, has been proposed by Angyal and Macdonald ${ }^{138}$. Here the difference in energy required to assume a greater degree of coplanarity for the $-\underset{1}{9}-C-$ groups of (98) and (99) is such that under normal conditions the O-isopropylidene derivative of a trans diol does not form. It should be noted, however, that complete coplanarity of the flve atoms of the 1,3-dioxolane type ring of O-isopropylidene derivatives is not, as was once supposed, necessary for their formation 139,140 . Additional evidence for the presence of two adjacent secondary hydroxyl groups having a c1s arrangement in one of the anhydrodeoxyheptitols was provided by its ready reaction with acetone, subsequent evidence showing that the mono-o-isopropylidene derivative so formed did not engage elther of the primary hydroxyl groups in the molecule. The results obtained could be interpreted in terms of the possible stereochemistry at $\mathrm{C}-2$ of 2,6-ahnydro-3-deoxy-heptitol B. 
The reaction of Fraction $B$ with acetone at room temperature, catalysed by a trace of sulphuric ac1d, gave an oll from which the reaction product was readily removed by extraction with boling carbon tetrachloride, in which the unreacted polyol was insoluble. The latter could then be subjected to further treatment with acidified acetone, thereby enabling the product to be obtained in good overall yield. The crystaline derivative, m.p. $104-105^{\circ},[\alpha]_{D}+12^{\circ}$, gave an elemental analysis in agreement with the emp/tycal formula $\left(\mathrm{C}_{10} \mathrm{H}_{18} \mathrm{O}_{5}\right)$ required for the mono-0-isopropylidene derivative, (100) of a 2,6-anhydro-3-deoxy-heptitol. The presence of two free hydroxyl groups was shown by conversion of (100)

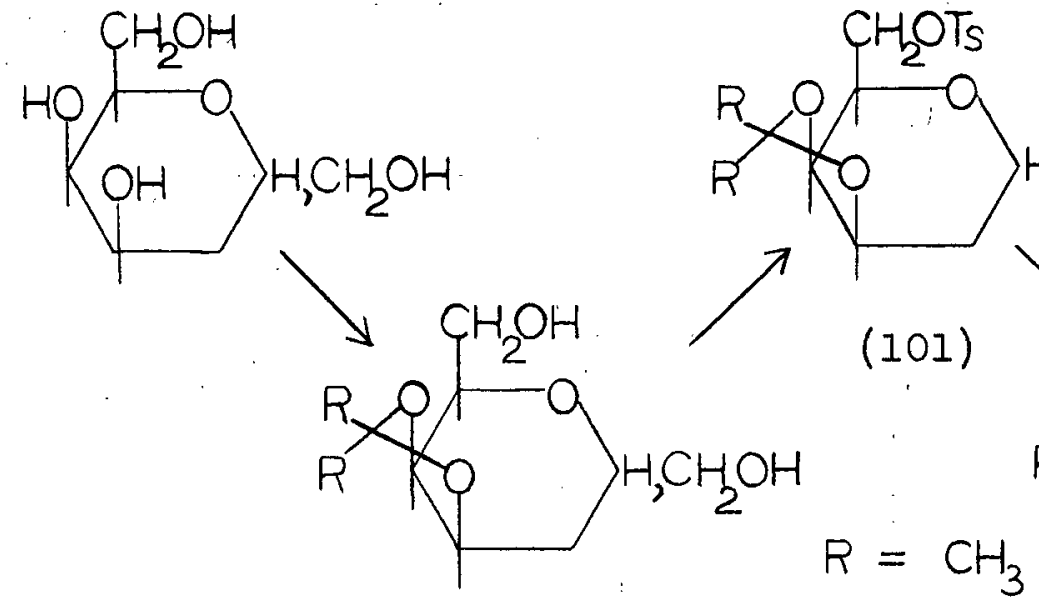

(100)

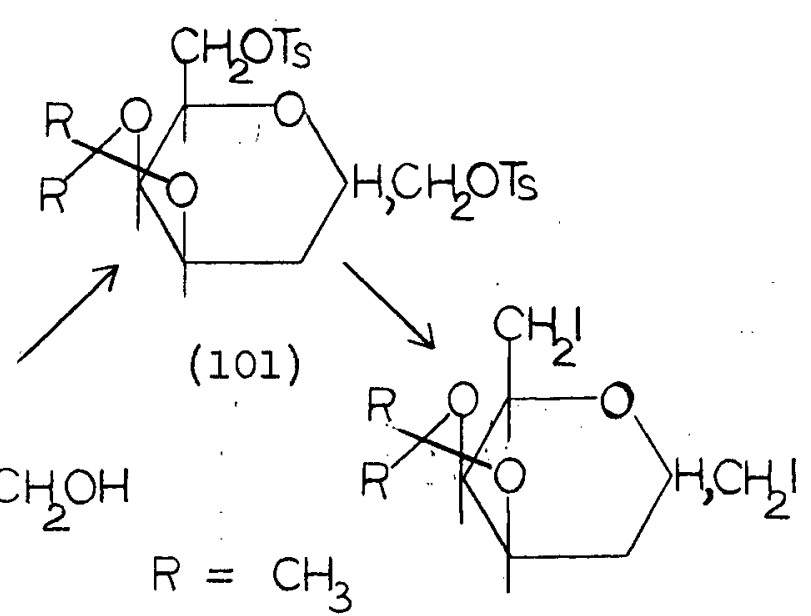

(102)

to a d1-0-p-ptolysulphonyl derivative (101), m.p. $135^{\circ},[\alpha]_{D}-42^{\circ}$. It was then possible to confirm that both hydroxyl groups of the mono-0-1sopropylidene derivative (100) not involved in acetal formation were primairy. When the di-0-p-tolysulphony 1 derivative (101) was heated in a sealed tube with sodium lodide 
In acetone solution for 26 hours at $118^{\circ}$, the amount of sodlum p-toluenesulphonate which was subsequently isolated by filtration was equivalent to the replacement of two tosyloxy groups by 1odide, to give the 1,7-dideoxy-derivative (102) (which was not obtained in sufficient amount for characterisation). It is well known that primary tosyloxy groups are replaced by lodide under these conditions, whereas those at secondary positions are generally unreactive 94 . These reactions therefore demonstrated the presence of two adjacent secondary hydroxyl groups having a cis relationship, in addition to two primary hydroxyls, in Fraction $B$.

In order to obtain the separation of an amount of sodium p-toluenesulphonate equivalent to the replacement of both primary tosyloxy groups of (101), it was necessary to employ a longer time and a higher temperature than is usual for this reaction. However, the formation of an appreciable amount of sodium p-toluenesulphonate was observed within minutes of the start of the reaction, whlle the temperature was still below $100^{\circ}$, and in a separate experiment carried out under the same conditions, but at a

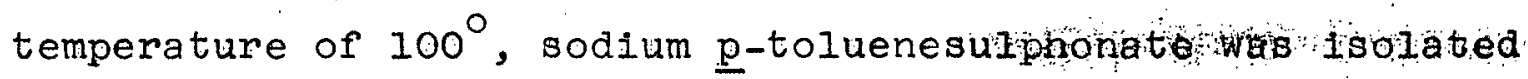
after 25 minutes equivalent to the replacement of one tosyloxy group by 1odide. It was apparent therefore, that whereas one of the primary tosyloxy groups of (101) was replaced by lodide w1th unusual ease, the other was replaced with difficulty. 
It has been shown that the proximity of an acetal ring can render an otherwise reactive primary tosyloxy group rather inert to the action of sodium lodide! Thus whereas 2,6-di-0p-tolylsulphonyl-D-galactose was readily converted to the 6-deoxy-6-iodo-derivative in good yield, the reaction of the corresponding 3,4-0-isopropylidene derivative (103) with sodium iodide under identical conditions resulted in only 18\% replacement of the primary tosyloxy group by lodide 141 . Similarly, replacement of the primary tosyloxy group in 1,2:
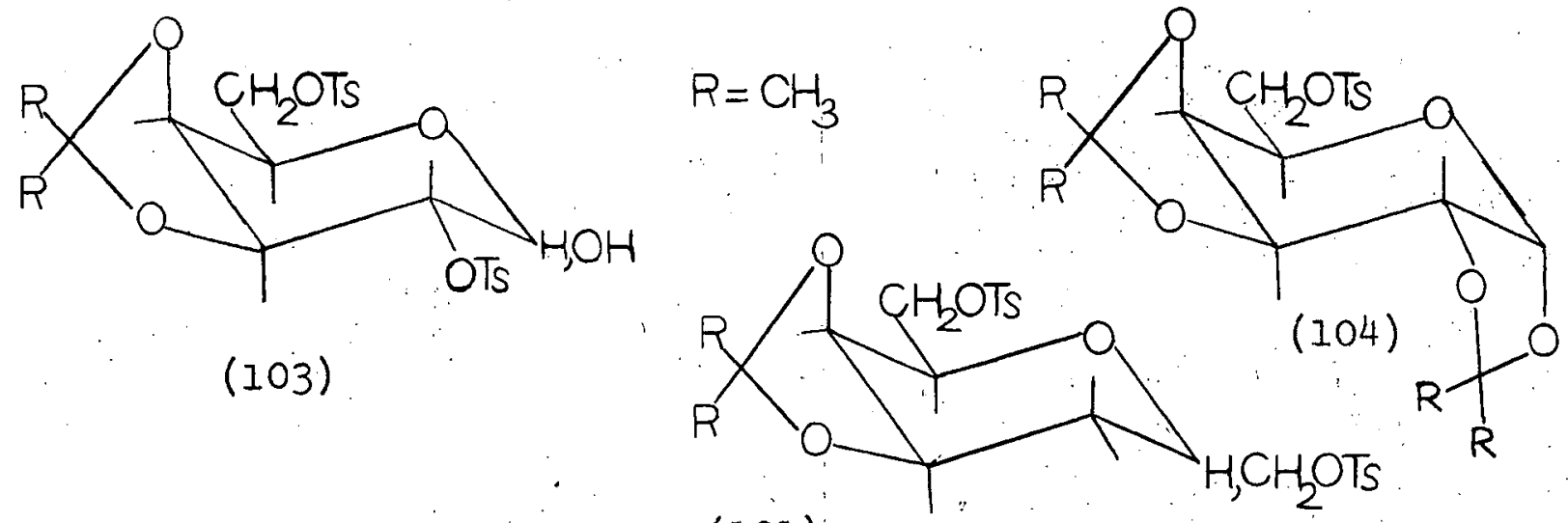

(101)

3,4-di-0-1 sopropylidene-6-0-p-tolylsulphonyl- $\propto-$ D-galactose (104) requires heating with sodium lodide in acetone for 36 hours at $125^{118}$. The structural similarity of (101) to (103) and (104) is apparent; hence, by analogy, it may be concluded that, of the two primary tosyloxy groups of (101), that at $\mathrm{C}-7$, adjacent to the acetal ring, was the one which reacted slowly with sodium lodide at $118^{\circ}$, and that the $\mathrm{C}-1$ tosyloxy group reacted very readily at $100^{\circ}$. 
It was then of interest to speculate on the likely orlentation of the reactive terminal tosyloxy group of (101), which must be ejther equatorial (in 2,6-anhydro-3-deoxy-4,5-0-1sopropylidene-1,7-d1-0-p-tolysulphony1-D-galacto-heptitol (105)) or axial (in the corresponding derlvative (106) having the talo- configuration).

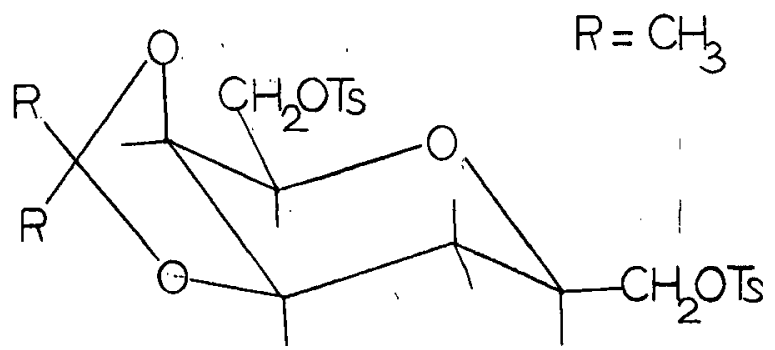

(105)

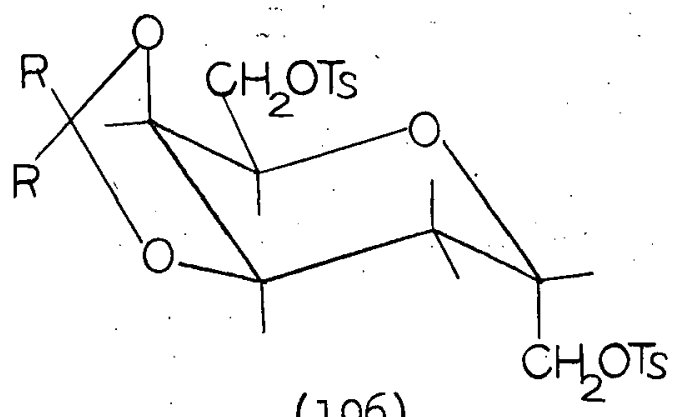

(106)

Evidence is avallable which demonstrates that an axialiy-disposed primary tosyloxy group will react with sodium Lodide with greater ease than an equatorial group, when both are in similar environments 142 . The bicyclic diacetals of hexitols, by cis fusion of two six-membered rings, give structures possessing two tehminal hydroxymethyl groups which can be axial or equatorial, depending on the conflguration of the parent hexitol. Thus 2,4:3,5-di-O-methylene-D glucitol (107), in the preferred conformation ${ }^{128}$, has the terminal C-6 group axial and the $c-1$ group equatorial. It has been shown that, on reaction of the 1,6-d1-0-p-tolylsulphonyl derlvative of (107) with sodium lodide, the axial 


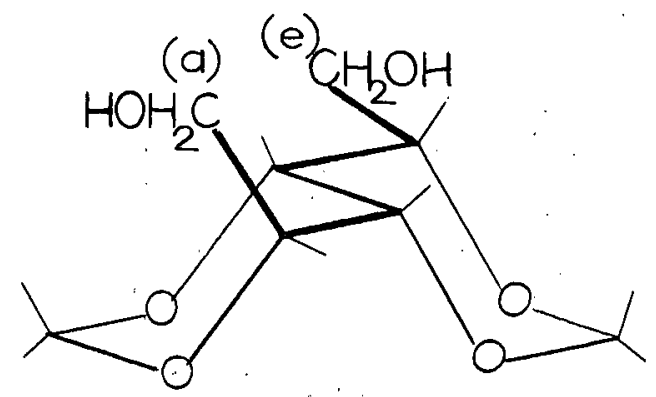

(107)

tosyloxy group at $\mathrm{C}-6$ was replaced more readily than the equatorial C-1 group ${ }^{143}$. Similarly, the replacement by lodide of the tosyloxy groups of 2,4:3,5-di-0-methylene1,6-d1-0-p-tolylsulphonyl-L-1ditol (both equatorial) was very slow at $100^{0144}$, whereas with the corresponding derlvative of D-mannitol, both (axial) tosyloxy groups reacted rapidly ${ }^{145}$. On the basis of these data, it was considered that (101) may have had the C-I tosyloxy group in an axial orientation (106), In which case Fraction B would be 2,6anhydro-3-deoxy-D-talo-heptitol (90). Subsequent evidence showed that this speculation was, in fact, correct. It would have been of interest to compare the same reactions of the corresponding derivatives of Fraction $A$; however, this compound, being sparingly soluble in acetone, did not form an 0 -isopropylidene derivative in sufficient amounts for characterisation and derivatisation. 
(b) Stereochemistry at $\mathrm{C}-2$

Attempts were made to solve the problem of the configurations of the enantiomeric tetrol ethers obtained from Fractions $A$ and $B$, (and hence the absolute stereochemistry of the two anhydrodeoxyheptitols), by the preparation of one of them from a suitable structure whose configuration at the potential asymmetric centre (c-3 of the tetrol ethers) was known (a procedure which had previously been successful in proving the structures of the anhydrodeoxyhexitols (44) and (45) by correlation with (59)). By the same approach, possible structures which would afford a 2-deoxy-3-0-(1,3-dihydroxy-2-propyl)-glycero-tetritol (109) were:

(a) 2,6-anhydro-3-deoxy-heptitols (108) other than Fractions $A$ and $B$, and

(b) 3,6-anhydro-2-deoxy-heptitols (I10), both by periodate cleavage of the $\mathrm{C}-4-\mathrm{C}-5$ bond and subsequent reduction. At the time this investigation was

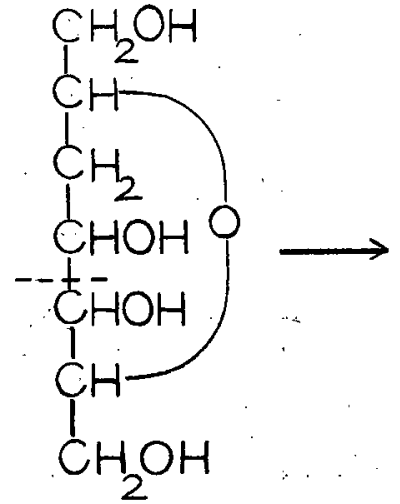

(108)<smiles>OCC(CO)OC(CO)CO</smiles>

(109)

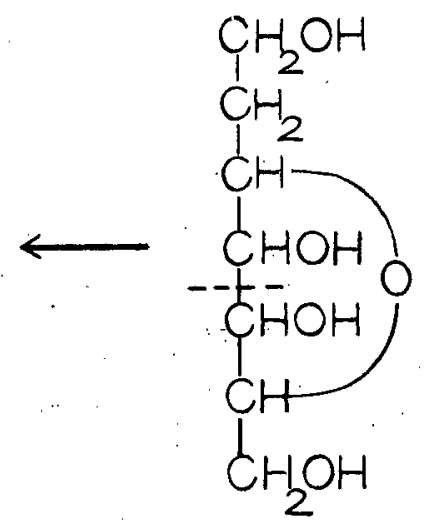

(110) 
undertaken no other compounds of the former type (108). were avallable whose stereochemistry had been established, and a search of the literature failed to reveal any known 3,6anhydro-2-deoxy-heptitols (110). However, it was considered that a possible synthetic route to the latter type of structure (110) would be the application to a 2,5-anhydrohexose (111) of a known procedure (the Fischer-Sowden nitromethane synthesis ${ }^{146}$ ) for the preparation of homologous 2-deoxy-aldoses, the product (112) on reduction then affording the required 3,6-anhydro-2-deoxy-heptitol (110), whose configuration at $\mathrm{C}-3$ would be the same as $\mathrm{C}-2$ of (111); thus

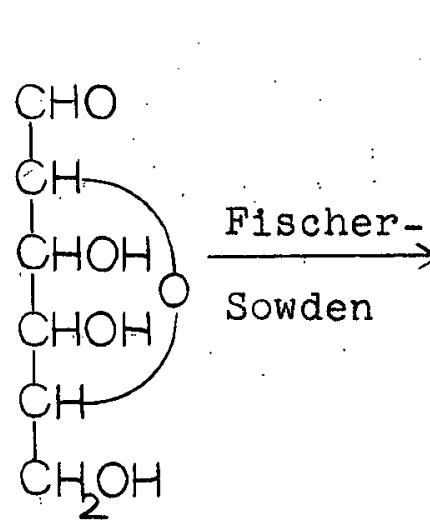

(111)

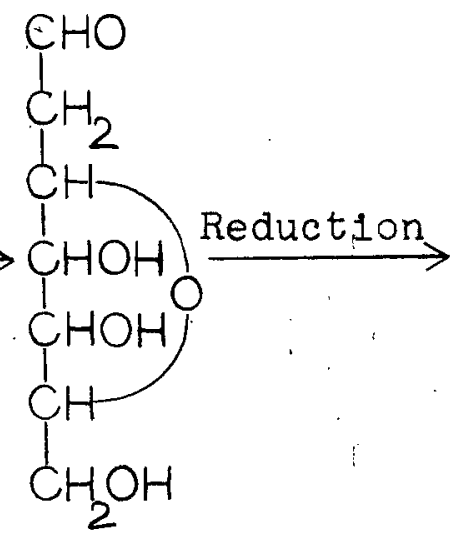

(110)

(112)

60

(c) Attempted Syntheses of 3,6-Anhdyro-2-deoxy-heptitols (110)

of the 2,5-anhydro-hexoses, the best known is 2,5anhydro-D-mannose (chitose)(115); an amorphous material, 2,5-anhydro-D-mannose has been known for many years 147 , but only comparatively recently has its structure been established 
beyond doubt ${ }^{148,149}$, confirming the previous conclusions of Levine and LaForge ${ }^{150}$. The formation of (115) by the nitrous acid deamination of 2-amino-2-deoxy-D-glucose (113) involves an inversion in configuration at $\mathrm{C}-2$, and is considered to proceed via an intermediate diazonium salt $(114)^{95}$; subsequent elimination is accompanied by rearward attack of the ring oxygen at $\mathrm{C}-2$, resulting in rearrangement of a six-to a five-membered ring. 2,5-Anhydro-D-glucose

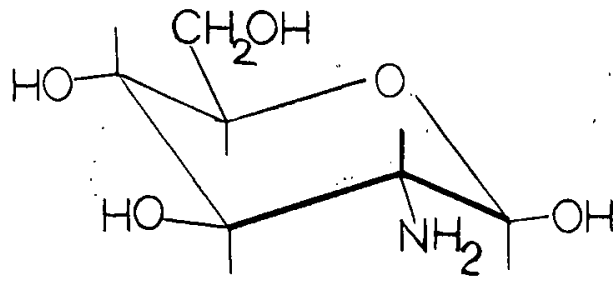

(113)

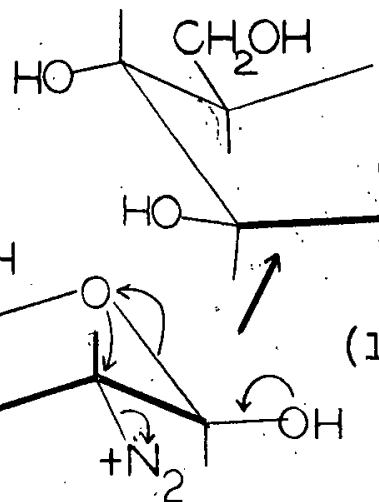

61

(114)

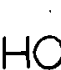

$\mathrm{HO}$

)
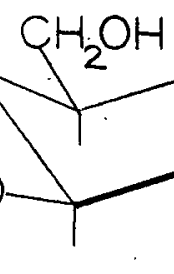

(117), the epimer of (115), is also known and has been obtained by the analogous deamination of 2-amino-2-deoxy-Dmannose hydrochloride (116) by the action of mercuric oxide ${ }^{151}$, again with inversion at $\mathrm{C}-2$

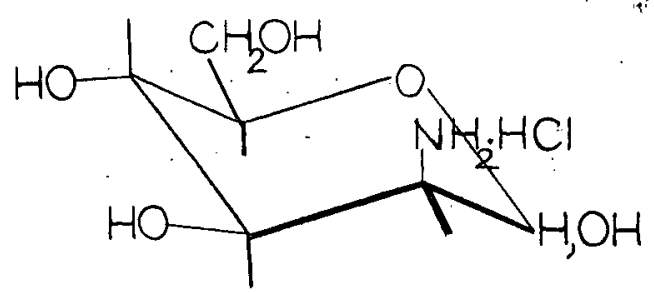

(116)

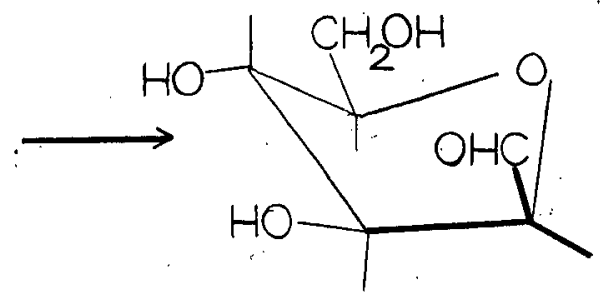

(117) 
Though less-readily available, 2,5-anhydro-D-glucose (117) was selected as being a more suitable starting material for the attempted preparation of a 3,6-anhydro-2-deoxyheptitol (110) than its epimer (115) for two reasons: (a) It is reported to be a highly-crystalline solid, whereas (115) is an 111-defined syrup, and (b) the preparation of its precursor, 2-amino-2-deoxy-D-mannose hydrochloride (116) from D-arabinose (118), according to Equation 63, would afford a series of intermedlate compounds ((119), (120) and (121)) which would provide useful models for following the subsequently planned conversion of (117) to 3,6-anhydro-2-deoxy-D-manno-hept1tol. (124), also via the nitromethane synthesis for 2-deoxy-sugars (Equation 64 ).

The preparation, described by Sowden and oftedahl 152 of 2-amino-2-deoxy-D-mannose hydrochloride (116) from $\stackrel{\text { D- }}{=}$ arabinose (118), by way of D-arabino-tetraacetoxy-1-nitro1-hexene (121) (Equation 63) proceeded smoothly, w1th formation of highly-crystalline intermediates. However, the attempted conversion of (116) to 2,5-anhydro-D-glucose (117), by the method of Levine 151 involving the action of mercuric oxide, did not give the anticipated crystalline anhydrosugar (117); an amorphous brown solid was obtalned which could not be purified. 
Attention was then turned to the use of the more accessible 2,5-anhydro-D-mannose (115); this was made by the method of Grant ${ }^{153}$, who investigated the stability of
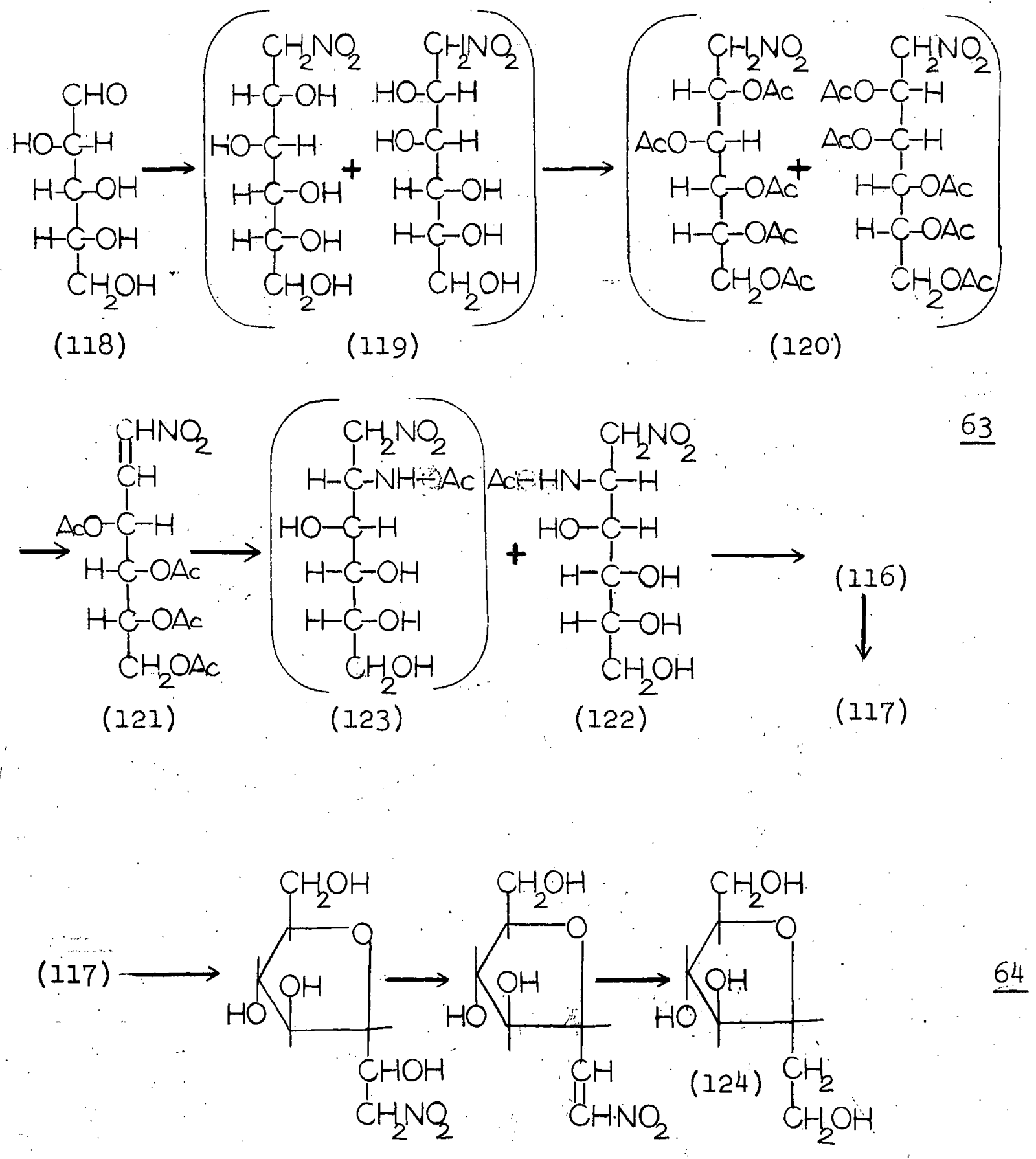
(115) under various conditions, and established optimum conditions for its preparation in a reasonably high state of purity. This was confirmed by the formation of a considerable yield of the p-nitrophenylhydrazone derivative of (115) from the syrupy product. When the anhydro-sugar (115) thus prepared was reacted with nitromethane in the presence of sodium methoxide under anhydrous conditions, according to the general procedure of Fischer and Sowden ${ }^{146}$, the immediate formation of a copious amount of white solid was observed, presumed to be the anticipated aci-sodium salts of nitroalcohols (125) and (126). Following deionisation by passage of an aqueous solution of the product

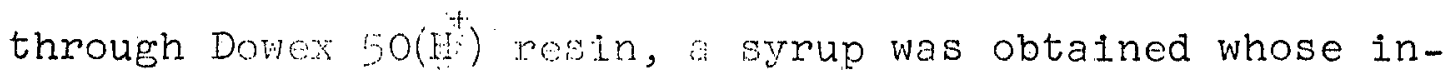
frared spectrum (Figure 4A) closely resembled that (Figure 4B) of the mixture (119) of 1-deoxy-1-nitro-D-mannitol and I-deoxy-1-nitro-D-glucitol previously obtained during the preparation of 2-amino-2-deoxy-D-mannose hydrochloride (116) (Equation 63). Infrared evidence, and the fact that thin layer chromatography of the product showed it to consist essentially of two closely ming components, indicated that the first stage of the attempted synthesis of 3,6-anhydro$(115)$

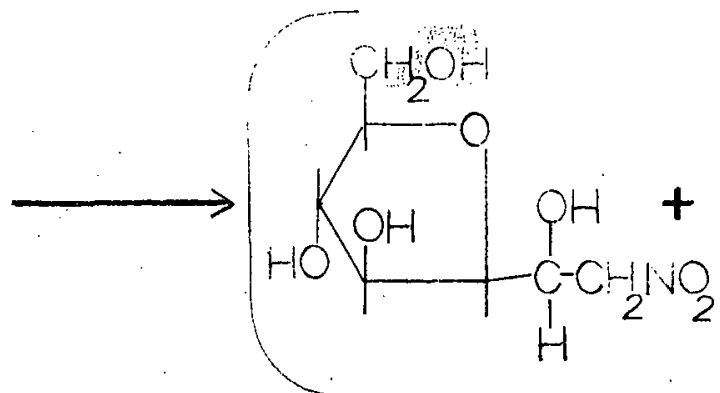

(125)

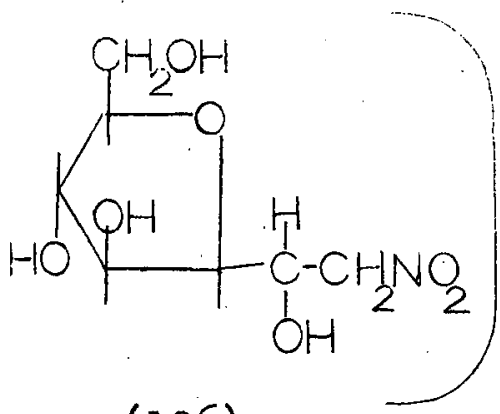

(126) 


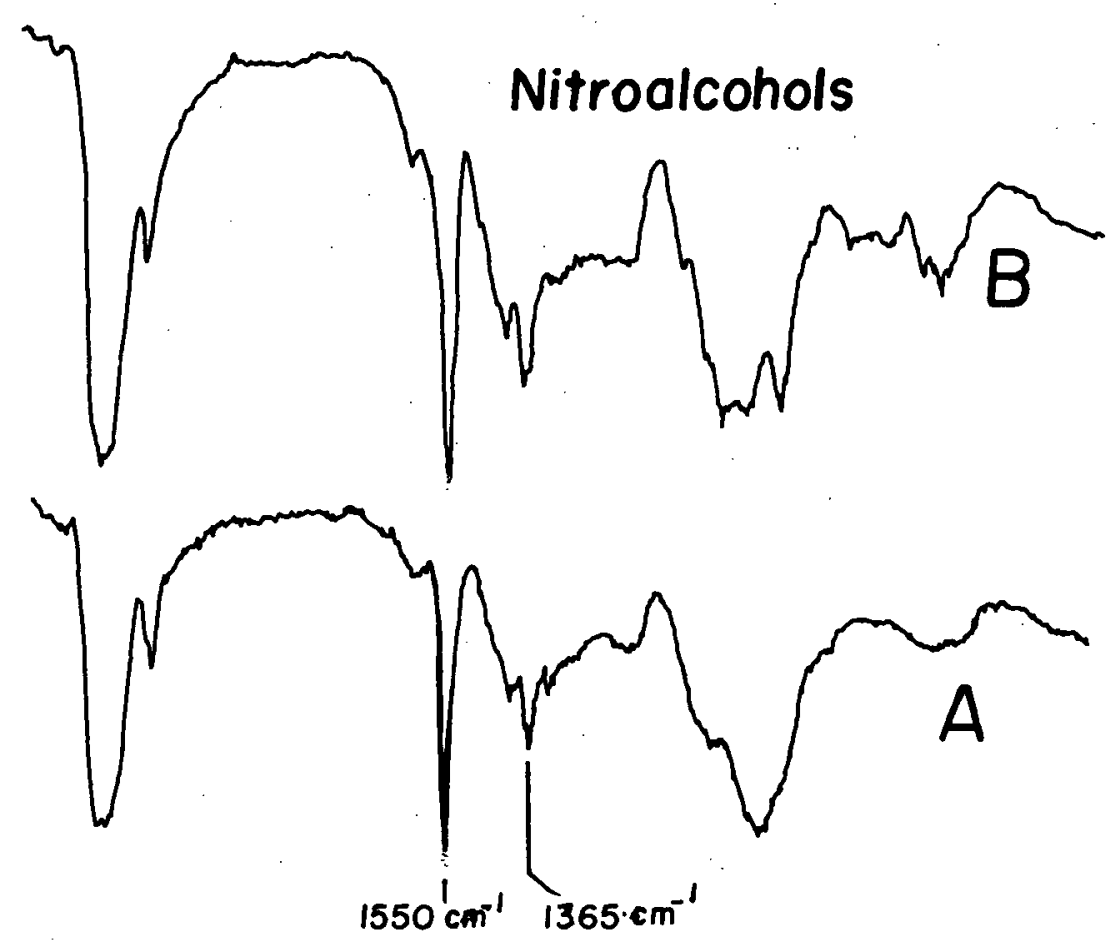

\section{Acetylated Nitroalcohols}
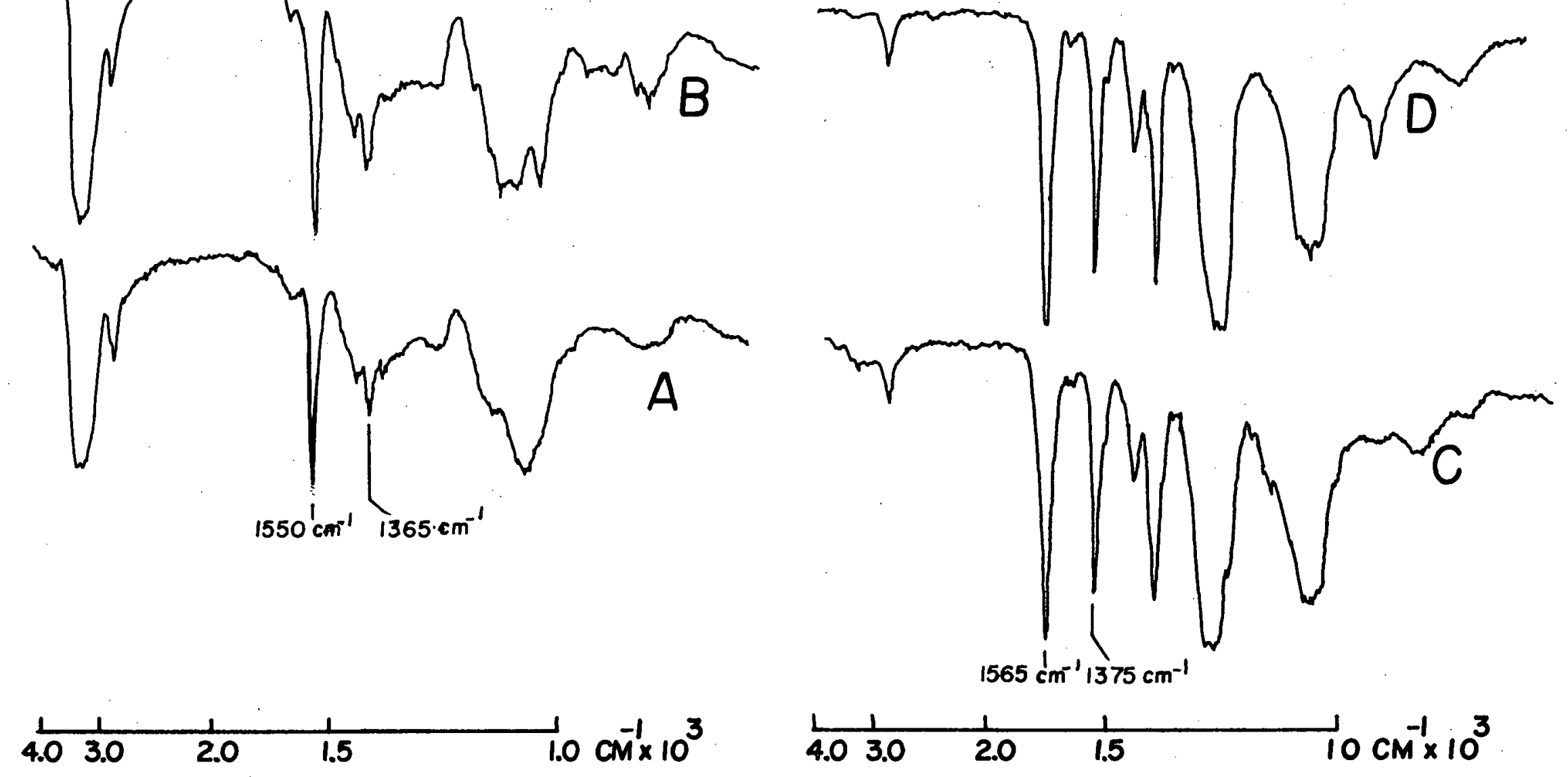

Infrared Spectra of: A and C - nitroalcohols and acetylated nitroalcohols from 2,5-anhydro-D-mannose

$B$ and D - nitroalcohols and acetylated nitroalcohols from $\stackrel{\text { D-arabinose }}{=}$

Figure 4 
2-deoxy-D-gluco-heptitol had gone as planned. Acetylation of the product with acetic anhydride containing a trace of sulphuric acid gave a mixture of acetates whose infrared spectrum (Figure 4C) also closely resembled that of the mixture (120) previously obtained by the acetylation of (119) (Figure 4D), showing absorption characteristic of $\mathrm{NO}_{2}$ stretching vibrations at 1550 and $1365 \mathrm{~cm}^{-1}$. When the acetylated material was refluxed in benzene solution with sodium hydrogen carbonate, the infrared spectrum of the product which was isolated was virtually unchanged from that of the acetylated reactant. Elimination of acetic acid, to give the required nitro-olefin, on treatment with mild base would presumably have been clearly apparent by a shift to lower frequencies of the asymmetric and symmetric stretching vibrations of the $\mathrm{NO}_{2}$ gfoup on conjugation $^{154}$, as was evidenced by the two bands at 1510 $\mathrm{cm}^{-1}$ and $1350 \mathrm{~cm}^{-1}$ in the infrared spectrum of D-arabinotetraacetoxy-1-nitro-1-hexene (121). Prolonged refluxing in benzene solution with sodium hydrogen carbonate, and similar treatment with the stronger base sodium acetate, failed to bring about the required elimination. This approach to the synthesis of a 3,6-anhydro-2-deoxy-heptitol, which apparently showed some promise in the earlier stages, was therefore abandoned as an alternative structural proof, described below, became available. 
(d) Correlation with 2,6-Anhydro-3-deoxy-D-gluco-hept1tol (130)

It was subsequently possible to establish the stereochemistry of one of the enantiomeric tetrol ethers previously described, and hence the absolute configurations of both Fractions $A$ and $B$, by correlation with the tetrol ether derived from a 2,6-anhydro-3-deoxy-heptitol (108) of known stereochemistry. Rosenthal and Koch ${ }^{155}$ have investigated the oxo reaction of the commercially-available 3,4,6-triO-acetyl-D-glucal (20 a). The products from this reaction were found to be completely a nalogous to those obtained from the reactions of 3,4,6-tri-0-acetyl-D-galactal (40) and 3,4-d1-0-acetyl-D-xylal (22). When the catalyst-free mixture of isomeric 4,5,7-tri-0-acetyl-2,6-anhydro-3-deoxyheptitols (127) and (128) resulting from the oxo reaction of (20 a) was reacted with p-bromobenzenesulphonyl bromide In pyridine, one of the isomers, (127), was readily isolated as the crystalline $\underline{0}-\underline{p}$-bromobenzenesulphonate. X-ray analysis of the crystaline derivative ${ }^{156}$ established that 1t was the 1-0-p-bromobenzenesulphonyl derivative (129) of 4,5,7-tr1-0-acety1-2,6-anhydro-3-deoxy-D-gluco-heptitol (127). Deacetylation of the catalyst-free oxo product and fractionation by paper chromatography gave two isomeric anhydrodeoxyheptitols. One of these, 2,6-anhydro-3-deoxyD-gluco-heptitol (130) was also obtalned from the 1-0-pbromobenzenesulphonyl derivative (129), of known configuration, 
by standard procedures

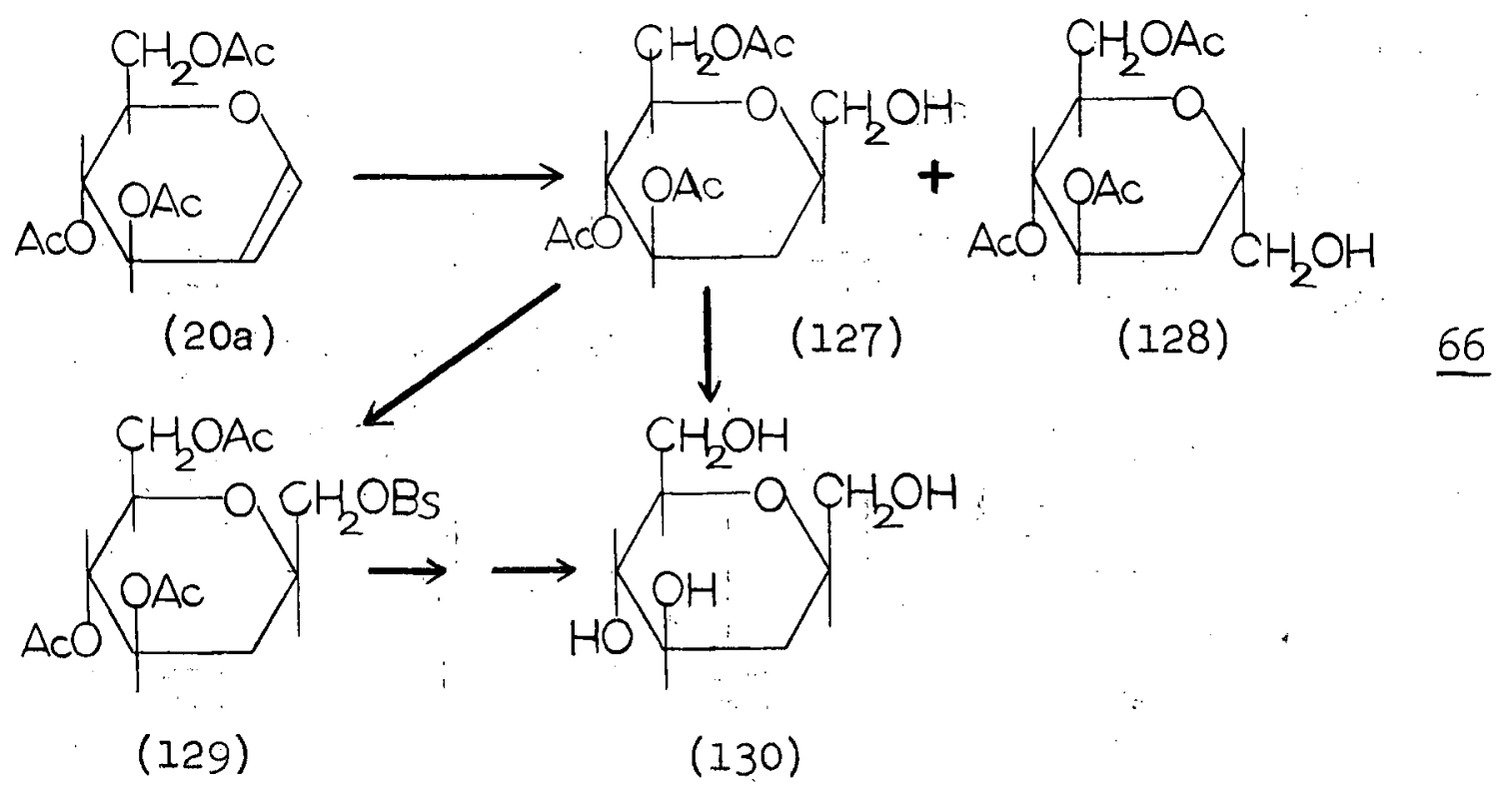

2,6-Anhdyro-3-deoxy-D-gluco-heptitol (130) was subjected to periodate oxidation and the resulting dialdehyde was reduced with sodium borohydride, using the procedure described previously, to afford 2-deoxy-3-0-(1,3dihydroxy-2-propyl)-L-glycero-tetritol (95). This was dextrorotatory, $\left([\alpha]_{D}+26^{\circ}\right)$, as was the tetrol ether

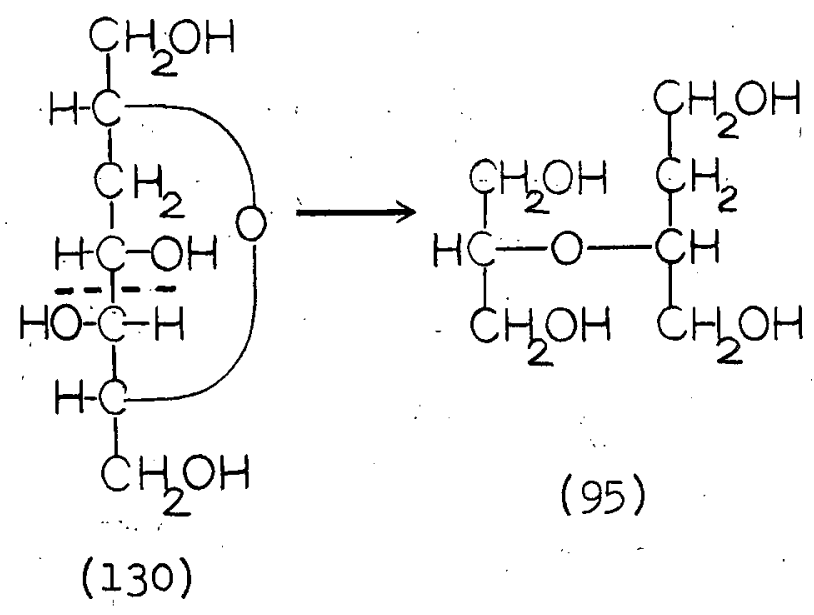




\section{- $117:$}

from Fraction A; (95) formed a tetra----nitrobenzoyl der1vative, m.p. $151-152^{\mathrm{c}}$, whose melting point was not depressed on admixture with the corresponding derivative of the tetrol ether from Fraction $A$, and the two p-nitrobenzoates had the same specific rotations and infrared spectra. Therefore Fraction A must have the D-configuration at $\mathrm{C}-2$, as had the D-gluco-isomer (130), this being the only asymmetric centre surviving in the tetrol ether (95) formed by cleavage of the $\mathrm{C}-4-\mathrm{C}-5$ bond and reduction. As 1 t could be safely assumed that the secondary hydroxyl groups at $\mathrm{C}-4$ and $\mathrm{C}-5$ of the anhydrodeoxyheptitols were unchanged in configuration from those of D-galactal, the structure of Fraction $A$ was therefore established as 2,6-anhydro-3-deoxyD-galacto-heptitol (91). As Fraction B differed from Fraction $\mathrm{A}$ only in configuratior at $\mathrm{C}-2$, this must be $2,6-$ anhydro-3-deoxy-D-talo-heptitol (90).

(v) Steric Aspects of Hydroxymethylation

The reactions of acetylated glycals with carbon monoxide and hydrogen to give, as major products, an approximately equal ratio of the two possible products resulting from addition of a hydroxymethyl group at C-1 of the glycal would appear to be general; this is supported by the fact that essentially similar results are obtained by the oxo reaction of 3,4,6-tri-0-acetyl-D-glucal (20 a $)^{155}$ and 3,4-di-0-acetyl-D-arabinal (32) 122 . That hydroxymethylation 
occurs at the carbon of the double bond $\propto$ to the ring oxygen of the glycals is consistent with the findings of other workers regarding the application of the oxo reaction to cyclic vinyl ethers such as 2,3-dihydro-4H-pyran (1) 25 and furan $(9)^{26}$. However, in view of the strong steric dependence of the oxo reaction when applied to olefins in general, and the apparent steric factors involved in add1tion reactions of glycals, both of which were discussed in the General Introduction, it is surprising that approximately equal amounts of two straight-chain products are always obtained, as a result of cis addition to both sides of the glycal double bond, rather than a preponderance of the isomer which would result from addition at the leasthindered side.

Heck and Breslow ${ }^{17}$ found that, at oxo reaction temperatures, the formation of alkylcobalt tetracarbonyls from olefin and cobalt hydrotetracarbonyl (Equations 7 - 9 ), in what is assumed to be the first stage of the oxo reaction, is rapidly reversible. These workers found that the product distribution obtained by reaction of olefins with cobalt hydrotetracarbonyl at low temperature was often not the same as that found under normal oxo conditions: for example, whereas isovaleraldehyde is the major product from the oxo reaction of isobutylene (equation 15 ), at low temperature trimethylacetaldehyde, resulting from addition to the more 
highly substituted side of the double bond, predominates. These findings were explaired by the fact that, as the Initial addition to the double bond is reversible, the product distribution at high temperature is a reflection of the relative stabilities of the adducts, rather than their initial concentrations, the terminal aldehyde being more stable yet forming less readily.

A similar explanation could account for the observed isomer distribution from the oxo reaction of glycals. Assuming that addition to the least-hindered side of the double bond is favoured, and that the first stage of the reaction leads to glycosylcobalt tetracarbonyls according to the generally accepted mechanism, it would be reasonable to suppose that the adduct so formed (e.g. (131) from 3,4d1-0-acetyl-D-xylal) would be less stable than the alternative, kinetically less-favoured adduct (132) with the $-\mathrm{Co}(\mathrm{CO})_{4}$ group in equatorial orientationfland would revert more readily to the glycal.

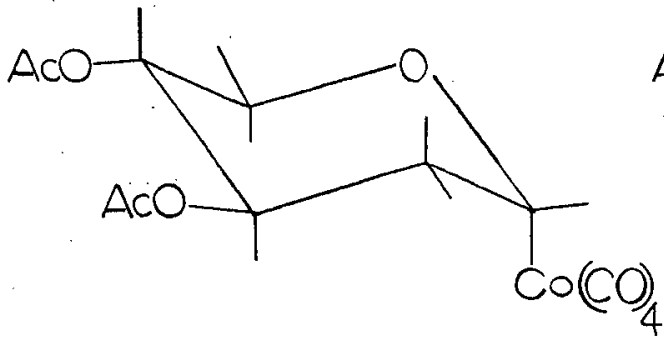

(131)

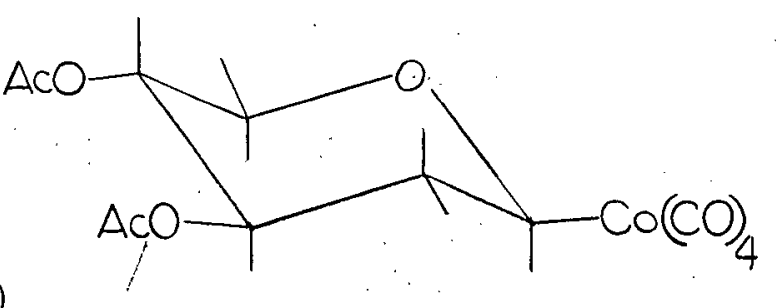

(132) 
C. Hydroformylation of 3,4-Di-O-acetyl-D-xylal

In section A it was shown that the reaction of $3,4-$ di-0-acetyl-D-xylal with 3 moles of synthesis gas gave a mixture consiting predominantiy of the 2,3-d1-0-acetyl-1,5anhydro-4-deoxy-hexitols (74) and (75), from which (44) and (45) were obtained on deacetylation. An a priori assumption regarding the oxo reaction of glycals is that the inftial products are aldehydes, resulting from the hydroformylation of the double bond, and that these subsequently undergo hydrogenation to afford the observed products. On this basis it was reasonable to assume that termination of the reaction at the point where only 2 moles of synthesis gas had reacted would give a product consisting predominantly of aldehydes; for example the anticipated products from the hydroformylation of 3,4-di-0-acetyl-Dxylal (22) would be the isomeric 4,5-di-0-acetyl-2,6anhydro-3-deoxy-aldehydo-hexoses (133) and (134).

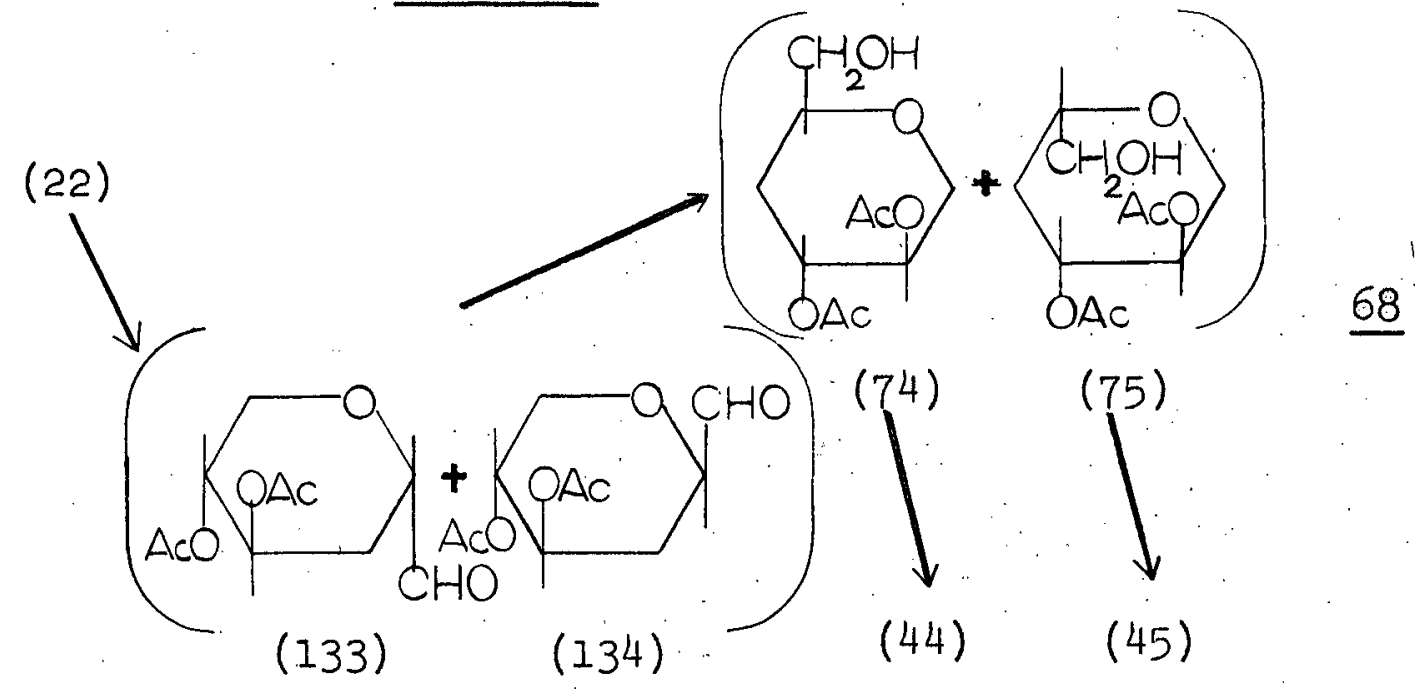


A certain amount of evidence has been obtained previously to show that aldehydic compounds are present in products obtained by the oxo reaction of glycals; for example, this has been indicated by the reducing power of reaction products to Somogy1's reagent ${ }^{157}$. From the reaction of 3,4,6-triO-acetyl-D-glucal (20 a) with carbon monoxide and hydrogen ${ }^{64}$, a single crystaline component was isolated, in addition to the acetylated alcohols (127) and (128), whteh was though to be an $\underline{0}$-acetylated-anhydrodeoxy-aldehydo-heptose, giving (130) on deacetylation and reduction. Additional evidence for the presence of an aldehydo-compound in the hydroformylation product of (20 a) has been obtained 158 on its reaction with ethyl mercaptan, as a homogeneous syrup was isolated by chromatography whose n.m.r. spectrum was consistent with an acetylated thioacetal.

\section{(1) Reaction Conditions and Product Isolation}

Conditions for the reaction of 3,4-di-0-acetyl-Dxylal with 2 moles of synthesis gas $((22) \longrightarrow(133)+(134)$, Equation 68) were similar to those previously described for its hydroxymethylation (Section A), except that a lower temperature $\left(115^{\circ}\right)$ was employed for the reaction, to favour the formation of aldehydes. Once absorption of synthesis gas commences, as evidenced by a decrease in the pressure Indicated on the guage attached to the reaction vessel, it rapidy overshoots the hydroformylation stage, and it was 
necessary to carry out a preliminary experiment under identical conditions in order to determine the point equivalent to the absorption of 2 moles of gas, when the reaction was quenched by immersion of the reaction vessel in ice.

The reaction product was isolated in a similar manner to that described previously. Catalyst was removed from the reaction mixture by elution from a Florisil column with petroleum ether. The fraction which was then eluted with benzene-1sopropyl alcohol welghed only $10.0 \mathrm{~g}$ (from $12.0 \mathrm{~g}$ of (22)) after evaporation of solvent and therefore did not represent the whole of the reaction product. A further $2.5 \mathrm{~g}$ of a mobile syrup was isolated from the petroleum ether eluate; this crystallised on standing, and was identified as 3,4-di-0-acetyl-D-xylal by comparison of its thin layer chromatogram and infrared spectrum against an authentic specimen of the glycal. It was apparent, on thin layer chromatography of the main fraction eluted from Florisil with benzene-isopropyl alcohol, that it also contained an appreciable quantity of the unreacted glycal, this being further indicated by a sharp peak at $1640 \mathrm{~cm}^{-1}$ in the infrared spectrum of this fraction. In addition to 3,4-di-o-acetyl-D-xylal, thin layer chromatography revealed the presence of a compact mixture of other components, (closely resembling in mobility and general appearance the mixture resulting from the hydroxymethylation 
of (22) (Section A)). This mixture of reaction products, belng well separated from the faster-moving glycal, was readily isolated by chromatography on a column of alumina. A portion of the main fraction thus separated gave 3,4di-O-acetyl-D-xylal and reaction products in the ratio of $2: 3$. Taking into account the additional amount of glycal eluted from Florisil along with the catalyst, the composition of the mixture obtained from the reaction was therefore approximately 50\% unreacted 3,4-d1-o-acetyl-Dxylal and an equal amount of saturated products.

Comparison of the reaction product, separated from unreacted glycal as described above, with the product previously obtained by the reaction of (22) with 3 moles of synthesis gas (Section A), showed that the two were practically indistinguishable on the basis of thin layer chromatograms and infrared spectra. However a difference was found in the low field region of the n.m.r. spectra, as only the product isolated from the above reaction showed a single peak at $\delta=9.35 \mathrm{ppm}$, characteristic of aldehydic protons 159 . On the assumption that this was due to the presence of compounds $((133)+(134))$, and that the remainder of the mixture comprised their reduction products $((74)+$ (75)), the intensity of the low field absorption relative to the combined intensities of the acetoxy-hydrogen signals In the region of $\delta=2.0-2.2 \mathrm{ppm}$ indicated that approximately $20 \%$ of aldehydo-compounds were present in the mixture 
Results of thin layer chromatography clearly precluded the possibility of effecting a separation of the mixture by chromatographic procedures.

(ii) Reaction wh 2,4 - initrophenylhydrazine

Confirmation of the presence of aldehydic components in the main fraction from the attempted hydroformylation reaction of 3,4-di-0-acetyl-D-xylal was sought by reacting a portion of the product with a test solution of 2,4-dinitrophenylhydrazine, which was preparef, in the usual way ${ }^{160}$, by dissolving the reagent in aqueous sulphuric acid and diluting with ethanol. Immediate precipitation of a yellow solid was indeed observed; this changed to an orange oil on standing. However, a control experiment in which pure 3,4-di-o-acetyl-D-xylal, known to be present in the hydro-, formylation product, was separately treated in the same way also resulted in the precipitation of an orange solid in considerable amount. Clearly, under these conditions, isolation of any derivatives resulting from the reaction with 2,4-dinitrophenylhydrazine of aldehydo-hexoses present in the mixture would have been complicated by the presence of derivatives originating from the glycal. The nature of the latter product was not investigated further, but is presumed to result from one or both of two known reactions of glycals under aqueous acid conditions, namely the addition 
of water across the double bond leading to 2-deoxy-aldoses ${ }^{39}$, and their ready isomerisation to pseudo-glycals ${ }^{161}$. Additional complications such as deacetylation would be anticipated under these strongly acid conditions, Indicated by the observed change from a solid to an oil.

Conditions were therefore established for reacting the hydroformylation product with 2,4-dinitrophenylhydrazine In the absence of strong ac1d. A solution of the reagent in ethanol, saturated at the bolling point, was added in portions to a solution of the reaction product in ethanol contalning a trace of acetic acid, also heated to the bolling point on a steam bath. Reaction was indicated by the fact that after each addition the orange colour faded to yellow; when the colour no longer faded after heating for several minutes, indicating that a slight excess of the hydrazine was present, water was added to turbidity. On standing, a pale yellow solid separated, which was readily isolated by filtration. Under the same conditions 3,4-di-o-acetylD-xylal did not react, and only unchanged 2,4-dinitrophenylhydrazine was isolated following dilution of the mixture with water.

Thin layer chromatography of the yellow solid obtalned from this reaction revealed the presence of two major components, together with traces of others. The two major products of the reaction, the faster moving of which will be 
designated Fraction $X$ and the slower, Fraction $Y$, moved very closely together on silica gel using a variety of developing solvents. The absence of colourless 1mpurities In the mixture was demonstrated on spraying thin layer chromatograms with a sulphuric-nitric acid reagent and heating. From an amount of $1.2 \mathrm{~g}$ of the main fraction from the oxo reaction, $0.4 \mathrm{~g}$ of 2,4-dinitrophenylhydrazones, consisting essentially of Fractions $X$ and $Y$, was isolated. On the assumption that $X$ and $Y$ were derivatives of isomeric d1-0-acetyl-anhydrodeoxy-aldehydo-hexoses (133) and (134), and that derivatisation was roughly quantitative, the welght of hydrazones isolated therefore indicated the presence of approximately 15\% of aldehydes in the mixture. The n.m.r. spectrum of the product obtained by reaction with 2,4-dinitrophenylhydrazine confirmed its composition as a mixture of two 2,4-dinitrophenylhydrazones of acetylated anhydrodeoxy-aldehydo-hexoses. The readily-assignable 161 low field signals due to $-\mathrm{NH}$ and protons of the dinitrophenyl group showed identical chemical shifts for both isomers except for the aromatic C -6 proton, which has a large splitting $(J=9-10 \mathrm{c} / \mathrm{s})$ by coupling with the ortho-hydrogen. A pair of overlapping doublets $(J=9 \mathrm{c} / \mathrm{s})$ at $\delta=7.87$ and $7.95 \mathrm{ppm}$ demonstrated the presence of a mixture of isomers, this also being shown by 3 acetate signals at high field. The doublet at $\delta=7.85 \mathrm{ppm}(\mathrm{J}=6 \mathrm{c} / \mathrm{s})$ was assignable to the $-\mathrm{N}=\mathrm{C}-\mathrm{H}$ group and confirmed that the 


$$
-127
$$

derivatives were of aldehydes rather than ketones?

(a) Separation of Fractions $X$ and $Y$

When the mixture of 2,4-dinitrophenyihydrazones was heated with a small volume of ethanol, partial solution of the mixture occurred, leaving a granular yellow solid which was not readily soluble in ethanol. Upon isolation and examination by thin layer chromatography, the insoluble portion showed only one zone corresponding to the slowermoving of the two components (Fraction $\mathrm{Y}$ ) present in the mixture. The material thus isolated crystallised readily from chloroform-hexane as fine yellow needles, m.p. 225$226^{\circ},[\alpha]_{D}-60^{\circ}$, and gave an elemental analysis in agreement with that required for a 2,4-dinitropheny ihydrazone of a 4,5-d1-0-acetyl-2,6-anhydro-3-deoxy-aldehydo-hexose ((133) or (134)). The n.m.r. spectrum of this fraction differed chiefly from that of the mixture in showing two acetate peaks of equal intensity $(\delta=2.13$ and $2.17 \mathrm{ppm})$; a doublet $(\mathrm{J}=9 \mathrm{c} / \mathrm{s})$ at $\delta=7.85 \mathrm{ppm}$, assignable to the C-6 proton of the 2,4-dinitrophenyl group, confirmed the homogeneity of the preduct. Thus, fractional solution of the mixture afforded a convenient procedure for the separation of Fraction $Y$ from Fraction $X$ in appreciable amounts, thereby facilitating its further examination. Treatment of a larger quantity $(6.8 \mathrm{~g})$ of the main fraction (from 
the reaction of (22) with 2 moles of synthesis gas) with 2,4-dinitrophenylhydrazone as described previously gave 2.4 $g$ of the mixed hydrazones, from which $0.9 \mathrm{~g}$ of pure Fraction $Y$ was obtained by trituration of the mixture with warm ethanol, and recrystallization of the insoluble portion.

Isolation of the other derivative, Fraction $X$, from the alcohol-soluble residue, which contained both Fractions $X$ and $Y$, presented a difficult problem because of the similar moblitties of the two fractions on chromatography. It was possible to isolate a small amount of the fastermoving component, $X$, sufficient for characterisation, by chromatography on thick plates of silica gel using the technique of multiple development, with chloroform as developing solvent. The derivative isolated in this way had m.p. $132^{\circ},[\propto]_{D}-16^{\circ}$, and also analysed satisfactorily

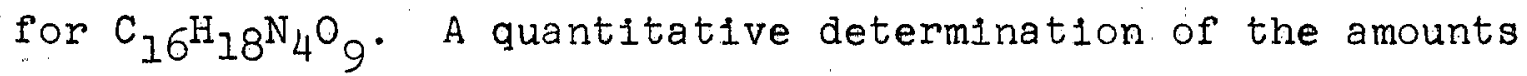
of the two isomeric 2,4-dinitrophenylhydrazones in the mixture, by chromatography on silica gel in the same way, showed that $X$ and $Y$ were present in the approximate ratio of $1: 2$.

(b) Identification of Fraction Y

An adequate amount of Fraction $Y$ being avallable, It was possible to convert this 2,4-dinitrophenylhydrazone to the free aldehydo-hexose, which was then identified by deacetylation and reduction to the corresponding anhydrodeoxyhexitol of known structure. Conversion of Fraction $Y$ 


\section{$-129$}

to the free aldehyde was effected by equilibration of the hydrazone with an excess of pyruvic acid, following a procedure applied by Mattox and Kendal1 162 to the hydrolysis of the 2,4-dinitrophenylhydrazone of a steroldal ketone. The 2,4-dinitrophenylhydrazone of pyruvic acid so formed was readily removed from the reaction mixture by filtration, and by extraction with sodium hydrogen carbonate solution.

A syrupy product was isolated from this reaction which was not completely characterised; however; the fact that it was an aldehydo-compound 159 was shown by the presence of a sharp signal at $\delta=9.35$ ppm in its n.m.r. spectrum, measured in deuterlochloroform solution. It is thought that the product probably existed as a mixture of free and hydrated aldehyde, as the intensity of this low fleld signal relative to the remainder of the spectrum was only about half that required for a 4,5-di_-abetyl-2,6-anhydro-3-deoxyaldehydo-hexose ((133) or (134)). Additional evidence for this was the presence in the infrared spectrum of bands assignable to both aldehyde ( $C=0$ stretching at $1700 \mathrm{~cm}^{-1}$ in addition to acetate absorption at $1740 \mathrm{~cm}^{-1}$ ) and hydroxyl groups. That the latter were not a result of deacetylation during and subsequent to the exchange reaction with pyruvic ac1d was shown by the fact that when a portion of the syrupy product was reacted with 2,4-dinitrophenylhydrazine, the original crystalline derivative, Fraction $Y$, was obtained. Wolfrom 
and co-workers 163,164 have observed the ready formation, by $0-$ acetyl-aldehydo-sugars, of crystalline hydrates and alcoholates, which have been shown to be aldehydrol (135) and hemlacetal. (136) derivatives; their formation accounts for the mutaro-

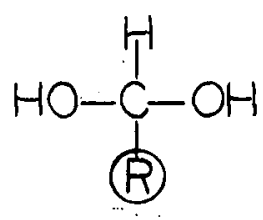

(135)
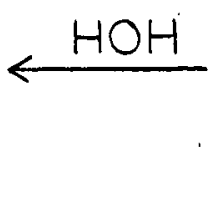

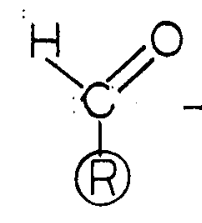

(R)

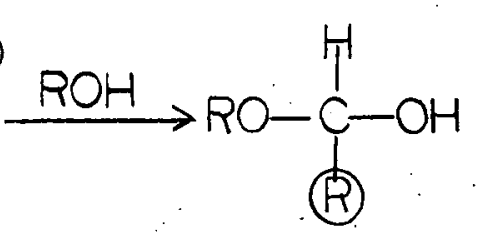

(136)

tation of aldehydo-sugars in aqueous and alcohollc solutions.

The aldehydic product thus isolated was readily 1dentified as 4,5-di-0-acetyl-2,6-a nhydro-3-deoxy-aldehydoD-1yxo-hexose (133). A portion was reacted with sodium borohydride in aqueous methanol, whereby the aldehyde group was reduced to hydroxymethyl, and simultaneous hydrolysis of the acetyl groups was effected in the basic medium. The product isolated from this reaction was subjected to chromatography on paper alongside control spots of the anhydrodeoxyhexitols (44) and (45), (Section A), and thereby identified with the faster-moving 1somer, 2,6-anhydro-3-deoxy-Darabino-hexitol (44). The aldehydic precursor therefore had the D-1yxo-configuration (133), and Fraction $Y$ was 4,5-d1-0acetyl-2,6-anhydro-3-deoxy-aldehydo-D-1yxo-hexose 2,4-dinitrophenylhydrazone. It followed that Fraction $X$ must be the corresponding derivative of 4,5-d1-0-acetyl-2,6-anhydro-3-deoxyaldehydo-D-xylo-hexose (134), as the other major component 
(45), previously 1solated from the hydroxymethylation of 3,4-d1-0-acetyl-D-xylal, had the I-xylo-configuration. Thus
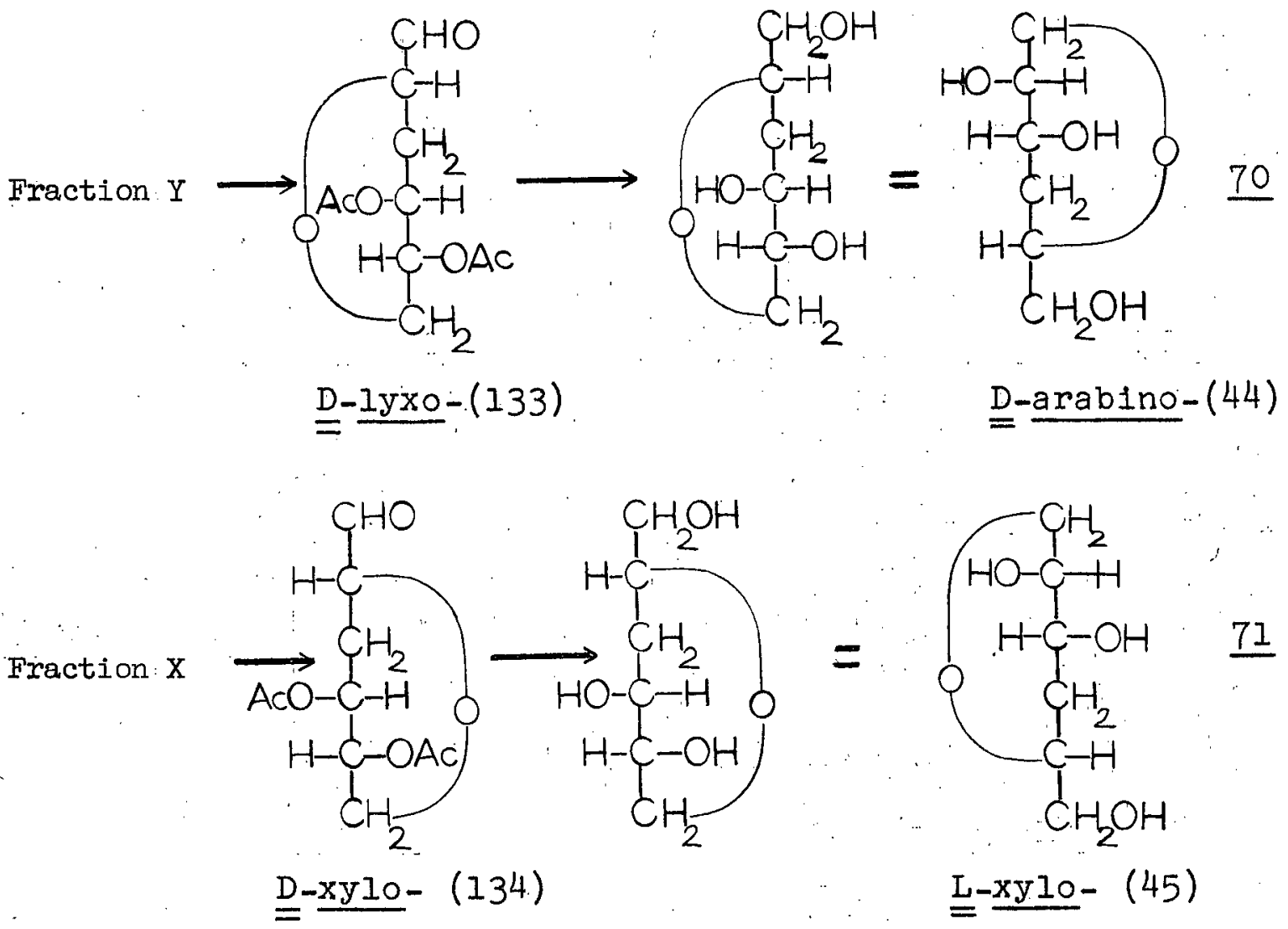

It is of interest that, whereas the anhydrodeoxyhexitols (44) and (45), previously isolated from the hydroxymethylation of 3,4-di-0-apetyl-D-xylal were present in nearly equal proportions, the amount of the D-Iyxo-hexose (133) isolated (as Fraction Y) from the intermediate stage of the reaction was considerably greater than the amount of its isomer (134). It has been shown that the rate of hydroformylation of olefins depends on the accessibility of the double bond ${ }^{16}$, and 1 it is reasonable to suppcse that a similar effect will operate in the hydrogenation stage, 
which is also considered to proceed via an intermediate $\pi$-complex with cobalt hydrotricarbonyl ${ }^{20}$. It is suggested therefore, that the carbonyl group of (134), being in equatorial orientation to the ring, is more accessible for complex formation, and (134) is more rapidly removed from the reaction mixture by hydrogenation that is (133), In which the formyl group is presumably axial.

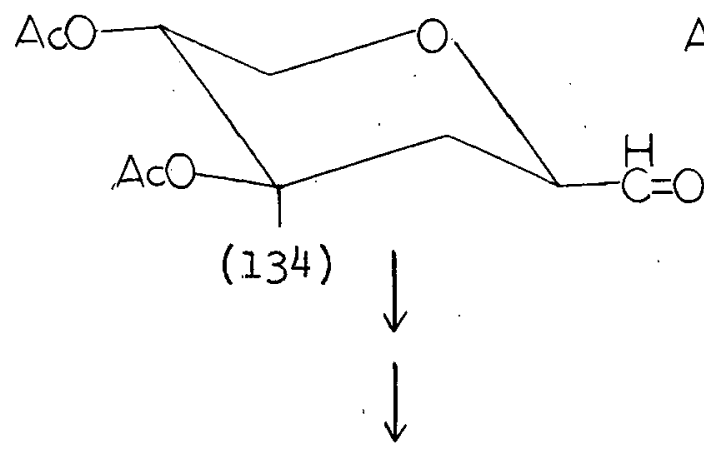

(45)

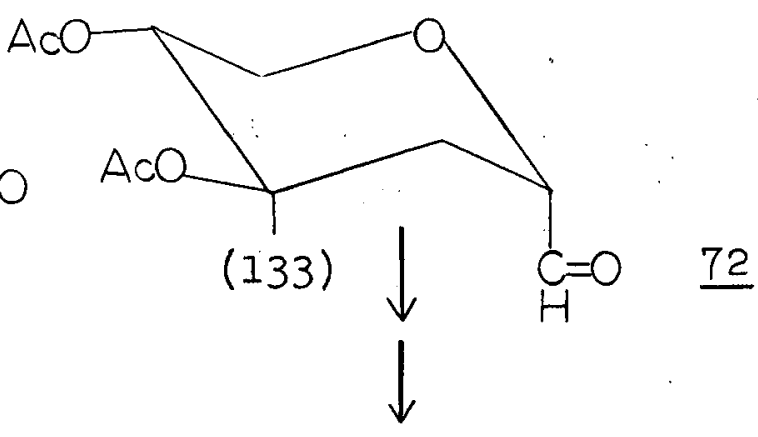

(44)

\section{(i11) Aldehydo-hexoses by Oxidation of Hexitols}

The attempted hydroformylation experiments described above clearly showed that the oxo reaction of 3,4-d1-0-acetylD-xylal was not.a satisfactory source of aldehydo-hexoses (133) and (134), as these were apparently too read1ly reduced to alcohols and did not accumulate in the reaction mixture. Some experiments were therefore carried out with a view to preparing (133) and (134) by oxidation of the more accessible mixture of d1-0-acetyl-anhydrodeoxyhexitols (74) and (75), resulting from the hydroxymethylation of (22) as described 
in Section A. Few methods are available for the oxidation of alcohols which stop short at the aldehyde stage; two recently described, and somewhat similar procedures which were investigated are those of Kornblum and co-workers and of Pfitzer and Moffatt ${ }^{166}$.

$$
\text { Kornblum and co-workers }{ }^{165} \text { have obtained good ylelds }
$$
of aldehydes by the oxidation of a variety of benzylic and straight chain aliphatic tosylates, on heating with a mixture of sodium hydrogen carbonate and dimethylsulphoxide. The limitations of the oxidation, which inftially involves displacement of the tosyloxy group by dimethylsulphoxide, with formation of an intermediate dimethylsulphoxonium salt ${ }^{167}$ were shown by the non-reaction of neopentyl tosylate. An attempt to oxidise the terminal tosyloxy group of a carbohydrate, 1,2-0-isopropyl1dene-6-0-p-tolysulphonyl-Dglucose, did not give the anticipated aldehydo-compound but the 5,6-carbonate 168 The mixture of d1-0-acetyl-1,5anhydro-4-deoxy-hexitols (74) and (75) comprising the hydrof ormylation product from (22) (Section A) was tosylated with p-toluenesulphonyl chloride in pyrldine to give the previously described crude mixture of isomeric 6-0- $-\underline{p}-$ tolylsulphonyl derivatives (76) and (77) (equation 52). This was then reacted for 5 minutes at $150^{\circ}$, in an atmosphere of nitrogen, with a mixture of sodium hydrogen carbonate and dimethylsulphoxide, according to the procedure 
of Kornblum and co-workers. From this reaction a dark coloured syrup was isolated whose infrared spectrum was virtually unchanged from that of the mixture of 6-0-ptolylsulphonyl derivatives $(76)$ and $(77)$.

Results of a more positive nature were obtained by the procedure of Pfitzer and Moffatt 166 which also involves the reaction of dimethylsulphoxide. These workers found that complex alcohols (nucleoside derivatives, steroids) were rapidly and selectively oxidised to the corresponding aldehyde (or ketone) on treatment with $\mathrm{N}_{,} \mathrm{N}^{\prime}$-dicyclohexylcarbodilmide (DCC) and dimethylsulphoxide at room temperature, in the presence of certain acids. A solution was prepared of the mixture of (74) and (75) (from the hydroxymethylation of 3,4-di-0-acetyl-D-xylal) in dimethylsulphoxide containing a trace of anhydrous phosphoric acid and an excess of DCC. After standing for one day at room temperature, during which time a crystalline precipitate of N,N'-dicyclohexylurea formed and dimethylsulphide was evolved, the mixture was filtered, diluted with ethanol and treated with a hot ethanolic solution of 2,4-dinitrophenylhydrazine, as described previously. On diluting with water a yeliow solid precipitated. This was collected by filtration and separated from co-precipitated $N, N^{\prime}$-dicyclohexylurea by solution in a small volume of chloroform, in which the urea was insoluble. Examination of the yellow product by thin layer chromatography 
revealed the presence of two major components, which were 1solated by chromatography on thick plates of silica gel, using multiple development with chloroform, and identified with Fractions $X$ and $Y$, the 2,4-dinitrophenylhydrazones of the D-xylo- and D-1yxo- isomers of 4,5-d1-0-acetyl-2,6anhydro-3-deoxy-aldehydo-hexose respectively, by melting point and mixture melting point, and thin layer chromatography .

The amount of mixed 2,4-dinitrophenylhydrazones obtained by this reaction was equivalent to the oxidation of approximately 35\% of the d1-0-acetyl-anhydrodeoxyhexitols (74) and (75), and comprised about $60 \%$ of the D-1yxo- ( $Y$ ) and about $30 \%$ of the D-xylo-derivative $(\mathrm{X})$. That the aldehydo-hexoses thus isolated were products of the oxidation reaction was confirmed by running separate control experiments under the same conditions, in one of which the hydroxymethylation product, and in the other the carbodilmide, were omitted. Ne1ther of these gave derlvatives on subsequent treatment with 2,4 -dinitrophenylhydrazine. 
EXPERIMENTAL

\section{General Considerations}

High pressure reactions were carried out using an Aminco $29 / 16^{\prime \prime} 0$. . Micro Series reaction vessel of manganese steel (Americal Instrument Co. Inc., Silver Spring, Md.). Infrared (I.R.) spectra were measured on PerkinElmer Model 21 and Model 137 (Sodium Chloride) spectrophotometers. Nuclear magnetic resonance (n.m.r.) spectra were recorded at $60 \mathrm{Mc} / \mathrm{s}$ on a Varian A60 spectrometer; resonance positions are recorded in ppm from tetramethylsilane as reference, set at zero (external with $D_{2} O$, internal with other solvents). Double resonance spectra were measured on a Varian D. P. spectrometer, also at 60 $\mathrm{Mc} / \mathrm{s}$, using a Heteronuclear Decoupler (Nuclear Magnet1c Resonance Specialties); modules operating at ca $9.2 \mathrm{Mc} / \mathrm{s}$ were used to provide the deuterium frequency. Gas-Iiquid partition chromatography (GLPC) separations were effected using an Aerograph "Autoprep" Model A-700 (W1ikens Instrument Co. Inc.), employing a column (10, $\mathrm{x} 1 / 4$ ") of $20 \%$ Silicone GE-SF-96 on firebrick, at a temperature of $180^{\circ}$, with helium as the carrier gas at a flow rate of $40 \mathrm{ml} /$ minute. Samples were infected directly onto the column using a 6" needle. Water-saturated 1-butanol containing 5\% ethanol at room temperature was employed as solvent for 
paper partition chromatography; $R_{F}$ values recorded are with reference to this solvent system. Polyols were detectedwith sodium periodate-Schiff reagent ${ }^{73}$. Thin layer chromatography (TLC) was on plates of Silica Gel G (acc. to Stahl), and zones were located by spraying with the general purpose reagent of sulphuric acid containing 5\% fuming nitric acid, and heating at $130^{\circ}$. Melting points were determined on a Kofler block and are uncorrected. Analyses were performed in the laboratories of Dr. A. Bernhardt, Mulheim (Ruhr), West Germany, and by the Microanalytical Laboratory, University of British Columbia. 


\section{Experimental Section A \\ 3,4-Di-0-acety1-D-xyla1 (22)}

In a $500 \mathrm{ml}, 3$-necked flask equipped with an efficient stirrer and a thermometer, acetic anhydride (200 ml) was cooled to $0^{\circ}$ and $70 \%$ perchloric acid $(1.2 \mathrm{ml})$ was added dropwise. The solution was then warmed to room temperature and $\underline{D}-x y l o s e ~(50 \mathrm{~g})$ was added in portions during the course of 1 hour to the stirred mixture at such a rate that the temperature did not rise above $30^{\circ}$. Red phosphorus ( $15 \mathrm{~g}$ ) was added after cooling the reaction mixture to below $20^{\circ}$ In an 1ce-water bath, followed by the dropwise addition of bromine $(30 \mathrm{ml})$ and then of water $(15 \mathrm{ml})$ to the continuously stirred mixture with control of temperature at or below $20^{\circ}$. After standing at room temperature for 3 hours the reaction mixture was filtered, and the filter paper was washed with a little glacial acetic acid. The deep yellow filtrate, containing 2,3,4-tri-0-acetyl- $\alpha-D-$ xylopyranosyl bromide, was immediately added to a reduction mixture, previously prepared as follows. A solution of socium acetate trihydrate $(200 \mathrm{~g})$ in water $(290 \mathrm{ml})$ and glaclal acetic acld (200 $\mathrm{ml})$ was cooled to $-10^{\circ}$, and zinc dust $(110 \mathrm{~g})$ and cupric sulphate pentahydrate (11 g) dissolved In water ( $40 \mathrm{ml}$ ) were added. When the blue colour had disappeared, the above solution of $2,3,4-t r i-0-a c e t y l-\alpha-D-$ 
xylopyranosyl bromide was gradually added over a period of $I$ hour to the mixture, which was maintained at $-10^{\circ}$ by cooling in an acetone-solid $\mathrm{CO}_{2}$ bath, and which was vigorously stirred by an efficient Hirschberg-type stirrer. Stirring was continued for a further 3 hours with the temperature at $-10^{\circ}$, Celite was added, and the mixture was filtered through a layer of celite into a suction flask containing ca $500 \mathrm{~g}$ of crushed ice, fragments of solid carbon dioxide being added to the mixture during filtration to prevent undue rise in temperature. After washing the filter with cold 50\% aqueous acetic acid (ca $150 \mathrm{ml}$ ), the filtrate was extracted with flve $100 \mathrm{ml}$ portions of cold chloroform. The combined chloroform extracts were washed with five $100 \mathrm{ml}$ portions of ice-cold water, cold aqueous sodium carbonate until free of acid, three $100 \mathrm{ml}$ portions of cold water, then dried over anhydrous calcium chloride, filtered and evaporated under reduced pressure to a syrup, which was immediately distilled under vacuum. The fraction b.p. 115-120\%/1.4 mm $(26 \mathrm{~g})$ crystallised overnight in the refrigerator; $[\infty]_{D}^{22}$ $-312^{\circ}$ (c, 2.3 in chloroform). One spot by TLC (benzenemethanoI, $96: 4 \mathrm{v} / \mathrm{v}$ ); I.R., strong band at $1640 \mathrm{~cm}^{-1}$ (C=C stretching).

\section{Dicobalt Octacarbonyl}

A slurry of cobalt (II) carbonate (15 g) in anhydrous benzene $(60 \mathrm{ml})$, contained in the Pyrex glass liner of a 
high pressure reaction vessel was shaken with carbon monoxide ( $1600 \mathrm{psi}$ ) and hydrogen ( $1600 \mathrm{psi}$ ) at $180^{\circ}$ for 2 hours. After cooling to room temperature the unreacted gases (combined pressure about 2400 psi) were vented and the dark brown solution was filtered to remove unreacted cobalt (II) carbonate. The filtrate was diluted with an equal volume of petroleum ether (b.p. $30-60^{\circ}$ ), and on standing at $-10^{\circ}$ In a well stoppered flask, orange crystals of dicobalt octacarbonyl were precipitated, yield about $10 \mathrm{~g}$. The crystalline product could be stored under the mother liquors at $-10^{\circ}$ for several weeks without undue decomposition.

\section{Hydroxymethylation of $3,4-d i-0-a \operatorname{cetyl-D-xyla1}$}

Typical experimental conditions for the absorption of 3 moles of synthesis gas were as follows. To a solution of 3,4-d1-0-acetyl-D-xylal (5.6 g) in anhydrous benzerie (25 $\mathrm{ml})$, contalned in the glass liner of a high pressure reaction vessel, was added dicobalt octacarbonyl $(1.5 \mathrm{~g})$. The stoppered liner was then inserted into the autoclave, which was flushed with carbon monoxide. Carbon monoxide was then added to a pressure of 500 psi, followed by hydrogen to a total pressure of 3000 ps1, and the reactants were heated, with rocking, at $125-130^{\circ}$ for about 2 hours. After cooling to room temperature overnight, unreacted synthesis gas pressure was released and the dark coloured solution was transferred 
to a short $(8 \times 8 \mathrm{~cm}$ diam.) column of Florisil, previously prepared as a slurry in anhydrous benzene. Elution with petroleum ether (b.p. $30-60^{\circ}$ ) was continued until catalyst was completely removed and the eluate was colourless, and the reaction product was then eluted with benzene-ethanol $(10: I, v / v)$. Evaporation of solvent under reduced pressure gave a syrup. $(6.0 \mathrm{~g})$ lconsifting principally of a mixture of 2,3-d1-0-acetyl-1,5-anhydro-4-deoxy-hex1tols.

To the syrupy product $(4.4 \mathrm{~g})$ dissolved in anhydrous methanol (50 ml) was added, with cooling, a solution of sodium $(0.2 \mathrm{~g})$ in anhydrous methanol ( $50 \mathrm{ml}$ ), and the mixture was set aside at about $5^{\circ}$ for 1 day. Fragments of solid carbon dioxide were then added until the solution was neutral to pH indicator paper, and solvent was removed under reduced pressure. The resulting solid residue was dissolved in water $(50 \mathrm{ml})$ and sodium ions were removed by passage of the solution through a column of Amberlite IR-120 $\left(\mathrm{H}^{+}\right)$ cation exchange resin, which was then washed with distilled water. The combined effluent and washings (total volume about $250 \mathrm{ml}$ ) were then reduced to a syrup $(2.75 \mathrm{~g})$ by evaporation under reduced pressure at about $45^{\circ}$. The product, which exhibited a strong band at around $3400 \mathrm{~cm}^{-1}$ (O-H stretching) In the infrared, showed two main components on paper chromatography. A portion $(0.40 \mathrm{~g})$ of the deacetylated mixture, dissolved in a small volume of methanol, was 
applied equally to si sheets $(57 \times 46 \mathrm{~cm})$ of Whatman No. I filter paper, prepared for descending chromatography. After equilibration and development for 40 hours, the two zones were located by spraying tests strips, cut from each sheet, with aqueous sodium periodate-Schiff reagent, and separately extracted with hot aqueous methanol $(I: I, v / v)$ to afford Fraction $I(0.15 \mathrm{~g}), \mathrm{R}_{\mathrm{F}} 0.47$, and Fraction II $(0.18 \mathrm{~g}), \mathrm{R}_{\mathrm{F}}$ 0.41 .

\section{Characterisation of Fractions I and II}

Fraction I (1,5-anhydro-4-deoxy-D-arabino-hexitol (44))

Fraction $I, R_{F} 0.47$, 1solated by paper chromatography, was dissolved in methanol, decolourised by filtration through a layer of Celite-Darco 60, and crystallised by the addition of 1sopropyl ether to incipient turbidity and ccoling in the refrigerator. Recrystalisation from the same solvents gave a product, m.p. $102^{\circ},[\alpha]_{D}^{20}-13^{\circ}$ (c, 6.3 in water). N.m.r. signals $\left(\mathrm{D}_{2} \mathrm{O}\right)$ : multiplet $3.2-4.2(7)$; multiplet $1.35-2.2 \mathrm{ppm}$ (2). Calc. for $\mathrm{C}_{6} \mathrm{H}_{12} \mathrm{O}_{4}: \mathrm{C}, 48.64 ; \mathrm{H}, 8.16$. Found: $\mathrm{C}, 48.35 ; \mathrm{H}, 8.10$.

$\frac{1,5 \text {-Anhydro-4-deoxy-2,3,6-tr1-0-p-n1trobenzoyl-D- }}{\text { arabino-hexitol }}$

Fraction I (3I mg) and p-nitrobenzoyl chloride (freshly distilled, $330 \mathrm{mg}$ ) were dissolved in anhydrous pyridine 
( $1 \mathrm{ml}$ ) and the solution was heated at $80-90^{\circ}$ for 1 hour. After cooling, the mixture was stirred with a saturated aqueous solution of sodium hydrogen carbonate ( $5 \mathrm{ml}$ ) for 30 minutes. The light coloured solid which separated was extracted with chloroform, and the extract was washed with saturated sodium hydrogen carbonate solution and with water, and dried over magnesium sulphate. Removal of solvent gave a syrup which crystallized from ethyl acetate-petroleum ether (b.p. $30-60^{\circ}$ ); m.p. $215^{\circ} ;[\alpha]_{D}^{20}-50^{\circ}$ (c, 1.0 in chloroform). Calc, for $\mathrm{C}_{27^{\mathrm{H}}} 21^{\mathrm{O}} 13^{\mathrm{N}}{ }_{3}$ : C, $54.60 ; \mathrm{H}, 3.60$. Found: C, $54.56 ; \mathrm{H}, 3.86$.

Fraction II (1,5-anhydro-4-deoxy-L-xylo-hexitol (45))

The component, $R_{F} 0.41$, was obtained as a syrup, after filtration through charcoal, which could not be crystallised. Purification by formation of a crystalline tri-O-acetyl derivative, as described below, followed by deacetylation with methanolic sodium methoxide, afforded a colourless syrup; $[\alpha]_{D}^{20}-44^{\circ}$ (c, 6.4 in water). N.m.r. signals $\left(\mathrm{D}_{2} \mathrm{O}\right)$ : multiplet $2.9-4.15(7)$; pair of quartets $1.75-2.2$ (1): multiplet $1.15-2.2 \mathrm{ppm}$ (1). Calc. for $\mathrm{C}_{6} \mathrm{H}_{12} \mathrm{O}_{4}: \mathrm{C}, 48.64 ; \mathrm{H}, 8.16$. Found: $\mathrm{c}, 48.36 ; \mathrm{H}, 8.39$. 2,3,6-Tri-0-acetyl-1,5-anhydro-4-deoxy-L-xylo-hexitel

Fraction II (70 mg) in pyridine $(1 \mathrm{ml})$ and acetic 
anhydride ( $1 \mathrm{ml}$ ) was heated for 20 minutes on a steam bath with exclusion of molsture. The solution was kept at room temperature overnight and then poured into ice-water (about $50 \mathrm{ml}$ ). After 1 hour the aqueous solution was extracted with chloroform, which was successively washed with $5 \%$ aqueous potassium hydrogen sulphate solution, saturated sodium hydrogen carbonate solution and water, and dried over magnesium sulphate. Removal of solvent gave a syrup (110 mg) which soon crystali1sod. Recrystalislation from ether-petroleum ether (b.p. $30-60^{\circ}$ ) afforded the pure triacetate; m.p. $80-81^{\circ} ;[\alpha]_{D}^{22}-41^{\circ}$ (c, 0.8 in chloroform). Calc. for $\mathrm{C}_{12} \mathrm{H}_{18} \mathrm{O}_{7}$ : C, 52.55; $\mathrm{H}, 6.62$. Found: C, 52.82; $\mathrm{H}, 6.58$.

Consumption of Periodate Ion ${ }^{76}$

\section{Fraction I}

Absorbance readings at $223 \mathrm{~m} \mu$ were measured at intervals on a Beckmann Model DU Spectrophotometer $(1 \mathrm{~cm}$ silica ceils) of an aqueous solution containing $0.439 \times 10^{-4} \mathrm{M}$ of Fraction I and $0.942 \times 10^{-4} \mathrm{M}$ of sodium periodate (Reading A). Simultaneous readings were made of a solution containing $0.439 \times 10^{-4} \mathrm{M}$ of Fraction I only (B), and of a solution containing $0.942 \times 10^{-4} \mathrm{M}$ of sodium periodate (C). The following values were obtained: 


$$
-145
$$

\begin{tabular}{l|c|c|c} 
T1me (hrs) & (B C) -A (a) & (B C) $-A^{(b)}$ & $\begin{array}{c}\text { Moles periodate/ } \\
\text { molell substrate }\end{array}$ \\
\hline 1.5 & 0.142 & 0.150 & 0.32 \\
3.5 & 0.207 & 0.218 & 0.47 \\
11.5 & 0.399 & 0.420 & 0.90 \\
22 & 0.381 & 0.401 & 0.86
\end{tabular}

(a): decrease in absorbance due to consumption of periodate (b): fraction of known amount of periodate consumed

\section{Fraction II}

In a similar manner, absorbances of solutions $0.453 \times 10^{-4} \mathrm{M}$ with respect to Fraction II and $0.942 \times 10^{-4} \mathrm{M}$ with respect to periodate ion were measured, to give the following values:

\begin{tabular}{l|l|l|l} 
Time (hrs) & $(\mathrm{B} C)-\mathrm{A}(\mathrm{a})$ & $\frac{(\mathrm{B} C)-\mathrm{A}(\mathrm{b})}{\mathrm{C}_{0}}$ & $\begin{array}{l}\text { Moles periodate/ } \\
\text { mole substrate }\end{array}$ \\
\hline 1.5 & 0.177 & 0.192 & 0.40 \\
3.0 & 0.263 & 0.284 & 0.59 \\
10.5 & 0.423 & 0.457 & 0.95 \\
24 & 0.392 & 0.424 & 0.88
\end{tabular}


Enantiomeric 2-deoxy-3-0-(2-hydroxyethyl)-glycero-tetritols

Fraction $I, R_{F} 0.47$ (47 mg) was dissolved in an aqueous solution $(5 \mathrm{ml})$ containing periodic acid ( $150 \mathrm{mg}, 50 \%$ excess) and 1mmediately transferred to a polarimeter tube which was protected from light. The optical rotation of the solution rapidiy assumed a constant value. After 6 hours the solution was removed from the tube, neutralised by the addition of excess barium carbonate, and filtered into a solution of sodium borohydride ( $50 \mathrm{mg}$ ) in water ( 3 $\mathrm{ml}$ ). After standing at room temperature for $11 / 2$ hours the solution was made slightly acid to $\mathrm{pH}$ indicator paper by the dropwise addition of acetic acid, Amberlite IR-120 $\left(\mathrm{H}^{+}\right)$resin $(5 \mathrm{ml})$ was then added, and the mixture was stirred for 1 hour. Filtration and evaporation under reduced pressure gave a solid residue, which was repeatedly evaporated with methanol to remove borate ion as the methyl ester. The resulting triol ether (III) (44 mg) was a syrup; $[\alpha]_{D}^{23}-19^{\circ}$ (c,2.0 in water); n.m.r. signals $\left(D_{2} O\right)$ : multiplet 3.5 - 3.9, with sharp signal at 3.72 (9); multiplet (apparent quartet) $165-2.0 \mathrm{ppm}$ (2).

The triol ether (III) was characterised as the trisp-nitrobenzoyl derivative: a portion (18 mg) was heated at $90^{\circ}$ with p-nitrobenzoyl chloride (160 mg) in anhydrous pyridine $(0.6 \mathrm{ml})$ for 1 , hour. Removal of excess p-nitro- 
benzoyl chloride and pyridine by stirring with saturated sodium hydrogen carbonate solution, extraction with chloroform, washing with sodium hydrogen sulphate solution, sodium hydrogen carbonate solution and water, drying over magnesium sulphate and removal of solvent gave a syrup ( $80 \mathrm{mg}$ ) which was crystalis ed from ethyl acetate-petroleum ether (b.p.

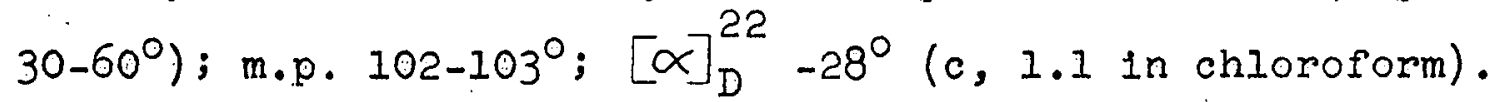
Calc. for $\mathrm{C}_{27} \mathrm{H}_{23} \mathrm{O}_{13} \mathrm{~N}_{3}: \mathrm{C}, 54.30 ; \mathrm{H}, 3.99$. Found: $\mathrm{C}$, 54.57; H, 3.95. The melting point of this derlvative was undepressed on admixture with the corresponding derivative of the triol ether obtained, by a similar procedure to the above, from the polyols isolated from the hydrogenolysis products of methyl $\propto-$ - $=$-glucopyranoside 82 .

Fraction $I I, R_{F} 0.41$, (30 mg) was converted by periodate oxidation and sodium borohydride reduction as described above to a triol ether (IV) (27 mg), which had $[\propto]_{D}^{25}+17^{\circ}$ (c, 1.7 in water); n.m.r. spectrum identical with that described above for triol ether (III) from Fraction I. A portion ( $13 \mathrm{mg}$ ) of triol ether (IV) from Fraction II was converted to a tris-p-nitrobenzoyl derivative on heating with p-nitrobenzoyl chloride $(0.13 \mathrm{~g})$ in pyridine $(0.5 \mathrm{ml})$, and Isolated in the usual way; the product (55 mg) on crystallisation from ethyl acetate-petroleum ether (b.p. 30$60^{\circ}$ ) had m.p. $102-103^{\circ} ;[\alpha]_{D}^{24}+26^{\circ}$ (c, 1.4 in chloroform); 
Infrared spectrum 1dentical with that of the tris-p-nitrobenzoate of triol ether (III). Caic. for $\mathrm{C}_{27} \mathrm{H}_{23} \mathrm{O}_{13} \mathrm{H}_{3}$ : C, 54.30; H, 3.99. Found: C, 54.63; H, 4.09.

$$
\frac{\text { 2-Deoxy -3-0-(2-hydroxyethy I) -I-glycero-tetritol (49) }}{\text { D-GalactaI (19) }}
$$

3,4,6-Tr1-O-acety1-D-galacta1 (40)(7 g) was dissolved In a solution (approximately $0.01 \mathrm{~N}$ ) of sodium methoxide In anhydrous methanol, and the solution was kept at room temperature for 48 hours. Removal of solvent under reduced pressure gave a syrup, from which D-galactal was isolated as white crystals on extraction with boiling ethyl acetate. Recrystallisation from the same solvent gave (19) (2.5 g), m.p. $100-102^{\circ}$.

Methyl 2-deoxy- $\propto$-D-galactopyranoside (55)

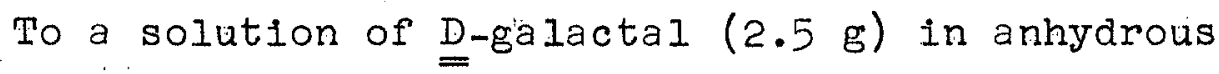
methanol $(22.5 \mathrm{ml})$ was added a $2 \%(\mathrm{w} / \mathrm{v})$ solution of hydrogen chloride in methanol $(2.5 \mathrm{ml})$. A portion of the solution was transferred to a $2 \mathrm{dm}$ polarimeter tube and the change in optical rotation was observed at intervals; no further change was observed after 90 minutes from the addition of hydrogen chloride. After 2 hours the recomblned solutions were neutralised by the addition of silver carbonate, filtered and evaporated to a syrup $(2.7 \mathrm{~g})$. Addition of a small volume 
of acetone resulted in the separation of crystals which were isolated and recrystallis ed from ethyl acetate; $1.0 \mathrm{~g} ; \mathrm{m} . \mathrm{p}$. $112-114^{\circ}$

Methyl 3,6-anhydro-2-deoxy- $\propto$-D-galactopyranoside (57)

To an 1ce-cold, stirred solution of methyl 2-deoxy$\alpha$-D-galactopyranoside $(1.0 \mathrm{~g})$ in anhydrous pyridine, a cooled solution of $\underline{p}$-toluenesulphonyl chloride $(1.0 \mathrm{~g})$ in anhydrous pyridine $(4 \mathrm{ml})$ was added over a perlod of 50 minutes, and the mixture was set aside at $0^{\circ}$ for 20 hours. Water $(0.5 \mathrm{ml})$ was added to the stirred solution at $0^{\circ}$, which after 30 minutes was then poured into 1ce-water (100 ml). The mixture was extracted with chloroform, and the combined extracts were washed with potassium hydrogen sulphate solution, sodium hydrogen carbonate solution and water, dried over magnesium sulphate, filtered and evaporated to a syrup (56) ( $1.46 \mathrm{~g})$. This was dissolved in ethanol (53 $\mathrm{mI})$; measurement of the optical rotation of this solution gave a value of $[\propto]_{D}^{23}+93^{\circ}$ (c, 2.6 in ethanol) for the 6-0-p-tolylsulphonyl derivative (56). To the recombined ethanolic solution was added IN sodium hydroxide solution $(4.3 \mathrm{ml})$, and the solution was heated at $60^{\circ}$ for 1 hour, neutralised with solid carbon dioxide and evaporated to dryness. The product was repeatedly extracted with boling acetone and after removal of solvent under reduced pressure 
the residue was redissolved in ethyl acetate. Filtration and evaporation of solvent gave a syrup $(0.65 \mathrm{~g})$, which distilled at $130^{\circ}$ (bath temperature)/0.1 $\mathrm{mm}$ as a colourless liquid which soon crystallised; yield $0.47 \mathrm{~g}$ of methyl 3,6anhydro-2-deoxy- $\alpha$-D-galactopyranoside (57), wh1ch was recrystallis ad from ether; m.p. $76-77^{\circ} ;[\alpha]_{D}^{24}+94^{\circ}$ (c, 5.7 in water).

$$
\text { 3,6-Anhydro-2-deoxy-D-1yxo-hexose (58) }
$$

To a solution of the methyl glycoside $(57)(0.28 \mathrm{~g})$ in water $(6.0 \mathrm{ml})$ was added $2 \mathrm{~N}$ sulphuric acid solution $(0.6$ $\mathrm{ml})$, and the mixture was left stand at room temperature for 110 minutes. Neutralisation with barium carbonate, filtration and removal of water by freeze-drying gave a colourless syrup, which was redissolved in water, filtered, and again evaporated by freeze-drying. The residue was dissolved in warm acetone, filtered and evaporated under reduced pressure to a clear, colourless syrup. Yield of 3,6-anhydro-2-deoxy-D-Iyxo-hexose, $0.26 \mathrm{~g} ;[\alpha]_{\mathrm{D}}^{25}+25^{\circ}$ (c, 5.1 in water). I.R. spectrum (Ilquid film): sharp peak at $1715 \mathrm{~cm}^{-1}$ (aldehyde $\mathrm{C}=0$ stretching).

1,4-Anhydro-5-deoxy-D-arabino-hexitol (59)

To a solution of the anhydro-sugar (58) (95 $\mathrm{mg}$ ) in water $(1.0 \mathrm{ml})$ was added dropwise a solution of sodium 
borohydride $(50 \mathrm{mg})$ in water $(1.0 \mathrm{ml})$. After 1 hour, Amberlite $I R-120\left(\mathrm{H}^{+}\right)$resin was added in small portions until Ihydrogen was no longer evolved; more resin (ca $3 \mathrm{ml}$ ) was then added and the mixture was stirred for 30 minutes. Filtration and evaporation under reduced pressure gave a solid residue which was repeatedly evaporated with methanol to afford 1,4-anhydro-5-deoxy-D-arabino-hexitol (59) as a colourless syrup (86 mg); $[\alpha]^{25}+21^{\circ}$ (c, 1.7 in ethanol). The anhydrodeoxyhexitol was characterised as the tris-pnitrobenzoyl derivative.

$$
\begin{aligned}
& \text { 1,4-Anhydro-5-deoxy-2,3,6-tri-0-p-n1trobenzoyl-D- } \\
& \underline{\text { arabino-hexitol }}
\end{aligned}
$$

A portion (25 mg) of (59) was heated with p-nitrobenzoyl chloride $(0.25 \mathrm{~g})$ in pyridine $(0.7 \mathrm{ml})$ and the product was 1solated in the usual way. The resulting syrup (114 mg) crystallised from chloroform-petroleum ether (b.p. $\left.30-60^{\circ}\right)$; m.p. $159-160^{\circ} ;[\alpha]_{\mathrm{D}}^{22}-96^{\circ}$ (c, 0.7 in chloroform). Calc. for $\mathrm{C}_{27} \mathrm{H}_{21} \mathrm{O}_{13} \mathrm{~N}_{3}$ : C, 54.46; H, 3.56. Found: C, 54.61 ; $\mathrm{H}, 3.86$.

2-Deoxy-3-0-(2-hydroxyethyl) - E-glycero-tetritol (49)

1,4-Anhydro-5-deoxy-D-arabino-hexitol (59) (55 mg) was dissolved in a solution of periodic acid ( $96 \mathrm{mg}, 1.3$ moles) 
in water $(5.0 \mathrm{ml})$. The solution was transferred to a polarimeter tube protected from light and its rotation was observed at Intervals and found to be constant after $31 / 2$ hours. After standing in the dark overnight the solution was neutralised with barium carbonate and then filtered into a solution of sodium borohydride $(70 \mathrm{mg})$ in water $(4 \mathrm{ml})$. After 2 hours the solution was neutralised with acetic acid, deionised by stirring with Amberlite $\mathrm{IR}-120\left(\mathrm{H}^{+}\right)$resin, filtered and evaporated under reduced pressure to a residue which was repeatedly evaporated with methanol to afford a syrup (5I mg), $[\propto]_{D}^{24}+17^{\circ}$ (c, 2.0 in water), whose n.m.r. spectrum $\left(\mathrm{D}_{2} \mathrm{O}\right)$ was identical with that described for triol ether (III), and with that of triol ether (IV).

A portion (30 mg) of (49) was converted to a trisp-nitrobenzoyl derivative in the usual way. Crystalisation from ethyl acetate-petroleum ether (b.p. 30-60\%) gave a product, $[\alpha]_{\mathrm{D}}^{24}+27^{\circ}$ (c, 1.1 in chloroform), whose melting point of $101-102^{\circ}$ was undepressed on admixture with the trisp-nitrobenzoate of the triol ether (IV) derived from Fraction II. The infrared spectra of both nitrobenzoates were identical. Calc. for $\mathrm{C}_{27} \mathrm{H}_{23} \mathrm{O}_{13^{\mathrm{N}} 3}$ : C, $54.30 ; \mathrm{H}, 3.99$. Found: C, $54.61 ; \mathrm{H}, 4.00$. 
Attempted preparations of 2-deoxy-3,4-d1-0-acety1-

D-xylopyranosyl cyanide

(a) By addition of HCN to 3,4-di-0-acetyl-D-xylal (22).

Hydrogen cyanide was generated by addition of a saturated solution of sodium cyanide to $50 \%$ sulphuric acid solution ${ }^{100}$; the gas was dried by passage through a series of calclum chloride tubes surrounded by water at $30-40^{\circ}$, and then condensed into a $50 \mathrm{ml}$ flask, protected from atmospheric molsture, containing 3,4-d1-0-acetyl-D-xylal $(0.52 \mathrm{~g})$ and sodium cyanide (10-15 mg), by passage through a coil surrounded by an ice-salt mixture. After the addition of about $5 \mathrm{ml}$ of $\mathrm{HCN}$, the flask was sealed by a calcium chlorlde tube, the solution was stirred at $0^{\circ}$ for 5 hours, and the hydrogen cyanide was then allowed to evaporate overnight. The I.R. spectrum of the residual syrup was unchanged from that of 3,4-di-0acetyl-D-Xylal.

(b) By reaction of 3,4-d1-0-acetyl-D-xylopyranosyl chloride with $\mathrm{Hg}(\mathrm{CN})_{2}$. 3,4-Di-0-acetyl-D-xylopyranosyl chloride (67): a cooled solution of 3,4-d1-0-acetyl-D-xylal $(2.5 \mathrm{~g})$ in anhydrous benzene ( $20 \mathrm{ml}$ ) was saturated with dry hydrogen chlorlde. After standing for 1 hour, the solvent was removed under reduced pressure at $30^{\circ}$ to give a colourless oil. 
I.R. spectrum (IIquid $f 1 I m$ ): disappearance of absorption at $1640 \mathrm{~cm}^{-1}$ (C=C stretching).

To a solution of $(67)(1.5 \mathrm{~g})$ in anhydrous nitromethane $(5 \mathrm{ml})$ was added mercuric cyanide $(1.7 \mathrm{~g})$, and the mixture was stirred at room temperature for 24 hours with exclusion of molsture. The dark coloured solution was reduced in volume under vacuum, methanol ( $15 \mathrm{ml}$ ) was added and the mixture was poured into water $(40 \mathrm{ml})$ and $1 \mathrm{ce}(30 \mathrm{~g})$ containing sodium chloride $(2.5 \mathrm{~g})$. Extraction with chloroform, washing with water, drying over sodium sulphate and evaporation of solvent gave a dark brown syrup, which was chromatographed through a column of neutral alumina using benzene-ethermethanol $(70: 30: 1, \mathrm{v} / \mathrm{v})$ as developing solvent to afford a nearly colourless syrup. T.L.C. (benzene-methanol, $96: 4$ $\mathrm{V} / \mathrm{v}$ ); complex mixture: I.R. spectrum (Iiquid film); no absorption in 2000-2300 $\mathrm{cm}^{-1}$ region $(\mathrm{C} \equiv \mathrm{N}): \mathrm{N}$, approximately $2.6 \%$.

In a simliar experiment, a solution of (67) ( $1.1 \mathrm{~g})$ in anhydrous benzene $(7 \mathrm{ml})$ was added dropwise to a refluxing suspension of silver cyanide $(0.7 \mathrm{~g})$ in anhydrous ether $(15 \mathrm{ml})$. After 5 hours the mixture was cooled, filtered and evaporated to a syrup which consisted of a mixture of unreacted (67) and 3,4-di-o-acetyI-D-xylal (identified by I.R. band at $1640 \mathrm{~cm}^{-1}$, and T.L.C.). 


2,3-D1-0-acetyl-1,5-anhydro-4,6-dideoxy-D-arabino-
hexitol (70) and 2,3-d1-0-acetyl-1,5-anhydro-4,6-
dideoxy-L-xylo-hexitol (71):

(a) By reaction of (67) w1th methyl magnesium bromide.

To a stirred solution of methyl magnesium bromide in dry ether, prepared by the slow addition of methyl bromide gas to a stirred suspension of magnesium $(3.2 \mathrm{~g})$ in dry ether $(50 \mathrm{ml})$, under an atmosphere of nitrogen, was added dropwise over 45 minutes a solution of 3,4-di-0-acetyl-2-deoxyD-xylopyranosyl chloride (prepared by the addition of hydrogen chloride to 3,4-d1-0-acetyl-D-xyla1 $(2.5 \mathrm{~g})$ as described above) In dry ether $(30 \mathrm{ml})$. After refluxing for 5 hours, the solution was cooled and added slowly to a mixture of ice and water (approximately $750 \mathrm{ml}$ ) containing acetic acid $(5 \mathrm{ml})$. The aqueous phase was filtered through Celite, neutralised with $2 \mathrm{~N} \mathrm{NaOH}$ solution, and evaporated to a white solid. The reaction product was separated from inorganic material by repeated extraction, filtration and evaporation with ethanol, and subsequently with acetone, to yleld a yellow syrup $(0.95 \mathrm{~g})$. Paper chromatography showed one zone $\left(R_{F} 0.69\right)$ on spraying with periodate-Schiff reagent. A portion of the product $(0.33 \mathrm{~g})$ was purified by chromatography on paper to give a mixture $(0.22 \mathrm{~g})$ of anhydrodideoxyhexitols (70) and (71). N.m.r. signals $\left(\mathrm{D}_{2} \mathrm{O}\right)$ : complex multiplet 


$$
-156-
$$

$3.0-4.2$ (5); multiplet up-field from 2.2 (2), $\mathrm{C}-\mathrm{CH}_{2}-\mathrm{C}$; pair of overlapping doublets $(\mathrm{J}=6 \mathrm{c} / \mathrm{s})$ at 1.14 and 1.17 ppm (3), $\mathrm{C}-\mathrm{CH}_{3}$.

A portion of the purified mixture of (70) and (71) $(100 \mathrm{mg})$ was acetylated with acetic anhydride ( $1 \mathrm{mI}$ ) and pyridine $(1 \mathrm{ml})$ at room temperature overnight. Isolation of the product in the usual way gave a syrup. (110 mg) which was fractionated by GLPC to yield two pure components (72) and (73), the latter being 1dentified as 2,3-d1-0acetyl-1,5-anhydro-4,6-dideoxy-L-xylo-hex1tol by independent synthesis from 1,5-anhydro-4-deoxy-L-xylo-hexitol (45) as described below.

$$
\text { 2,3-Di-o-acetyl-1,5-anhydro-4,6-aideoxy-D-arabino- }
$$

hexitol (72): relative retention time 1.10; colourless liquid; $[\alpha]_{D}^{24}-82^{\circ}$ (c, 1.0 in chloroform). Calc. for $\mathrm{C}_{10} \mathrm{H}_{16} \mathrm{O}_{5}: \mathrm{C}, 55.54 ; \mathrm{H}, 7.46$. Found: $\mathrm{C}, 55.61 ; \mathrm{H}, 7.55$. N.m.r. signals $\left(\mathrm{CCl}_{4}\right)$ : multiplets at $4.75-5.0(2), 4.4$ 4.65 (1), 3.35 - 3.9 (3); two sharp signals at 2.02 (3) and 2.06 (3), $-\mathrm{OCOCH}_{3}$; multiplet $1.5-1.85$ (2), $\mathrm{C}-\mathrm{CH}_{2}-\mathrm{C}$; doublet $(J=6 \mathrm{c} / \mathrm{s}) 1.13 \mathrm{ppm}(3), \mathrm{C}_{-} \mathrm{CH}_{3} \cdot$ 2,3-Di-0-acetyl-1,5-anhydro-4,6-dideoxy-I-xy10-hexitol

(73): relative retention time 1.00; colourless liquid; $[\alpha]_{D}^{24}-21$ (c, 0.9 in chloroform). Calc. for $\mathrm{C}_{10} \mathrm{H}_{16} \mathrm{O}_{5}: \mathrm{c}$, 
55.54; H, 7.46. Found: C, 55.47; H, 7.42. N.m.r. signa is $\left(\mathrm{CCI}_{4}\right)$ : multiplets at $4.65-5.0(2), 3.8-4.2$ (1) and $3.0-$ 3.65 (3); sharp signal at $1.97(6),-\mathrm{OCOCH}_{3}$, superimposed on multiplet (2), $\mathrm{C}-\mathrm{CH}_{2}-\mathrm{C}$; doublet $(\mathrm{J}=6 \mathrm{c} / \mathrm{s}) 1.20 \mathrm{ppm}$ (3), $\mathrm{C}-\mathrm{CH}_{3}$.

(73) from 1,5-anhydro-4-deoxy-L-xylo-hexitol (45)

To an ice-cold solution of (45) (70 mg) in anhydrous pyridine $(0.5 \mathrm{ml})$ was added over 20 minutes a solution of p-toluenesulphonyl chloride ( $105 \mathrm{mg}, 1.1$ moles) in anhydrous pyridine $(1.0 \mathrm{ml})$. After 18 hours at $5^{\circ}$, acetic anhydride $(1.0 \mathrm{ml})$ was added and the mixture was stood for a further 12 hours at room temperature. Isolation of the product in the usual manner gave a syrux (140 mg) which contained approximately $80 \%$ of 2,3-di-0-acetyl-1,5-anhydro-4-deoxy6-0-p-tolylsulphonyl-L-xylo-hexitol (77), as judged by the amount of sodium p-toluenesulphonate liberated therefrom. The crude product in acetone $(2 \mathrm{ml}$ ) was heated with sodium lodide ( $140 \mathrm{mg}$ ) in a sealed tube at $100^{\circ}$ for 3 hours. After cooling, sodium p-toluenesulphonate $(58 \mathrm{mg})$ was removed by filtration and the solution was evaporated under reduced pressure to a solid residue Extraction with refluxing ether and evaporation of solvent gave a syrup (125 mg). The crude 6-deoxy-6-iodo-derivative (79) was dissolved in methanol (10 $\mathrm{ml}$ ) containing $5 \mathrm{~N}$ sodium hydroxide solution $(0.5 \mathrm{ml})$, and 
shaken with hydrogen at atmospheric pressure and temperature in the presence of Raney nickel (15 mg) for 30 minutes. Filtration, neutralisation with solid carbon dioxide and evaporation under reduced pressure gave a white solid, which was acetylated by heating at $100^{\circ}$ for 3 hours with acetic anhydride $(2 \mathrm{ml})$ and anhydrous sodium acetate $(0.4 \mathrm{~g})$. Isolation of the product in the usual way gave 2,3-di-o-acetyl1,5-anhydro-4,6-dideoxy-L-xylo-hexitol (73) as a syrup (50 $\mathrm{mg}$ ), which showed one zone on GLPC whose retention time was identical with the faster-moving component of the mixture of acetates prepared as described above. The Infrared spectrum of a portion of the product thus purified by GLPC was identical with that of the latter zone, which was thus identified as the L-xylo isomer (73).

(b) From (74) and (75) obtained by reaction of 3,4-di-oacetyl-D-xylai with carbon monoxide and hydrogen

3,4-Di-o-acetyl-D-xylal $(5.6 \mathrm{~g})$ in anhydrous benzene $(25 \mathrm{ml})$ was reacted with carbon monoxide (1100 psi) and hydrogen (2200 psi) at $130^{\circ}$ for 3 hours in the presence of dicobalt octacarbonyl $(1.5 \mathrm{~g})$, and the product was separated from catalyst as previously described to give a syrup (6.I g) consisting chiefly of two isomeric 2,3-di-0-acetyl-1,5-anhydro4-deoxy-hexitols (74) and (75). A portion of the product $(1.5 \mathrm{~g})$ was deacetylated with methanolic sodium methoxide. 
Isolation in the usual way and fractionation by preparative paper chromatography gave 1,5-anhydro-4-deoxy-D-arabinohexitol (44) and 1,5-anhydro-4-deoxy-L-xylo-hexitol (45), identical with the fractions obtained in a previous experiment.

To a further portion of the oxo product $(1.5 \mathrm{~g})$ in anhydrous pyridine $(6 \mathrm{ml})$ was added p-toluenesulphonyl chloride $(1.8 \mathrm{~g})$. After standing at room temperature for 18 hours the product was isolated in the usual manner to yield a syrup $(1.6 \mathrm{~g})$ whose n.m.r. spectrum indicated approximately $75 \%$ of $2,3-d i-\underline{0}-a c e t y 1-1,5$-anhydro-4-deoxy-6-0- - (ㅁtolylsulphonyl)-hexitols $((76)+(77))$ to be present. The product $(1.5 \mathrm{~g})$ in acetone $(12 \mathrm{ml})$ was heated with sodium lodide $(1.5 \mathrm{~g})$ at $100^{\circ}$ for 3 hours in a sealed tube. After cooling, sodium p-toluenesulphonate $(0.52 \mathrm{~g})$ was removed by filtration, the filtrate was evaporated to dryness and repeatedly extracted with boiling ether. The crude mixture of 2,3-d1-0-acetyl-1,5-anhydro-4,6-dideoxy-6-1odo-hexitols $((78)+(79))(1.0 \mathrm{~g})$ was dissolved in methanol (40 ml) containing $5 \mathrm{~N}$ sodium hydroxide solution $(2 \mathrm{ml})$, and shaken with hydrogen at atmospheric pressure and temperature in the presence of Raney nickel. (100 mg) for 30 minutes, when $a b-$ sorption of hydrogen $(46 \mathrm{ml})$ was complete. After standing at room temperature for 2 hours the solution was filtered, neutralised with solid carbon dioxide and evaporated to a white solid. Repeated extraction, filtration and evaporation with 
acetone gave a pale yellow syrup, which was further purified by preparative paper chromatography to yield a colourless syrup $(0.20 \mathrm{~g})$; one zone on paper chromatography of 1dentical $R_{\mathrm{F}}$ value $(0.69)$ to the mixture $((70)+(71))$ previously obtained by reaction of (67) with methyl magnesium bromide. N.m.r. spectrum $\left(\mathrm{D}_{2} \mathrm{O}\right)$ : essentially identical with that described above for the mixture of anhydrodideoxyhexitols (70) and (71).

A portion of the purified product (110 mg) was acetylated with acet1c anhydride-sodium acetate to yleld a syrup (140 mg) which was fractionated by GLPC into (74) and (75), identical with the two fractions isolated as described above on the basis of retention times, specific rotations $\left([\alpha]_{D}^{24}\right.$ $-81^{\circ}$ and $-23^{\circ}$ respectively (c, 1.2 in chloroform)), and n.m.r. and infrared spectra.

Identification of "Polyol $Y$ " from Hydrogenolysis of Methyl $\alpha$-D-glucopyranoside 80,82

The tris-p-nitrobenzoate of this compound (170 mg), kindly supplied by Dr. P. A. J. Gorin, was debenzoylated by refluxing with $0.1 \mathrm{~N}$ methanolic sodium methoxide (30 ml) for 1 hour. Filtration to remove methyl p-nitrobenzoate, and isolation of the product in the usual way gave a colourless syrup (38 mg); $[\alpha]_{D}^{22}+40^{\circ}$ (c, 2.2 in water), (previously reported value $e^{82}$ was $\left.+19^{\circ}\right)$. N.m.r. signals $\left(b_{2} 0\right)$ : multiplet $2.9-4.15(7)$; pair of quartets centered around 2.0 
(1); multiplet ca $35 \mathrm{c} / \mathrm{s}$ wide centered around $1.5 \mathrm{ppm}$ (1). The spectrum was identical with that of 1,5-anhydro-4deoxy-L-xylo-hexitol (45).

2,3,6-Tr1-0-acetyl-1,5-anhydro-4-deoxy-D-xylo-hexitol

The anhydrodeoxyhexitol obtained on debenzoylation as described above was acetylated at room temperature for 18 hours with acetic anhydride ( $1 \mathrm{mI}$ ) and pyridine ( $1 \mathrm{ml}$ ). Isolation of the product gave a syrup which crystallised

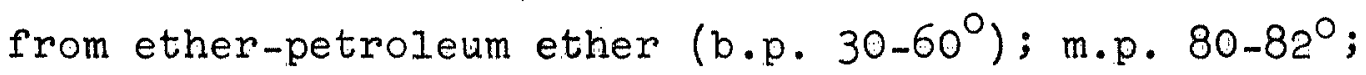
$[\propto]_{D}^{22}+40^{\circ}$ (c, 2.0 in chloroform). The infrared spectrum of the product was 1dentical with that of the tri-0-acetyl derivative of the levorotatory L-1somer (45).

Reaction of 3,4-Di-o-acetyl-D-xylal with Carbon Monoxide and Deuterium

3,4-Di-O-acetyI-D-xylaI ( $1.8 \mathrm{~g})$ in anhydrous benzene $(8 \mathrm{ml})$ was reacted with carbon monoxide (1000 psi) and deuterium ( $800 \mathrm{ps}$ ) at $130^{\circ}$ for $3^{1 / 2}$ hours in the presence of dicobalt octacarbonyl $(0.4 \mathrm{~g})$. After cooling overnight, unreacted gases were vented, and catalyst was removed by filtration through a short column of Florisil as described previously, to afford a $\operatorname{syrup}(1.8 \mathrm{~g})$ on removal of eluting solvent under reduced pressure. A portion $(0.8 \mathrm{~g})$ of the product was deacetylated with methanolic sodium methoxide 
and on working up in the usual way a $\operatorname{syrup}(0.56 \mathrm{~g})$ was isolated whose infrared spectrum showed the presence of deuterium (C-D stretching in $2200-2300 \mathrm{~cm}^{-1}$ region). Paper chromatography showed 2 main components of simllar mobilities to (44) and (45), chromatographed alongside as controls. Fractionation of a portion (0.48 g) of the $\mathrm{mlx}-$ ture of deuterated hexitols by paper chromatography gave the two components, which were separately purified by further chromatography on paper. The two pure fractions were subjected to n.m.r. analysis as described below.

$$
\text { 1,5-Anhydro-4-deoxy-D-arabino-hexitol-4,6,6- } \mathrm{H}_{3}^{2}(83)
$$$$
R_{F} 0.46 ;[\alpha]_{D}^{23}-11^{\circ} \text { (c,3.I in water). N.m.r. }
$$
signals $\left(D_{2} O\right)$ : multiplet $3.3-4.07(5)$; unresolved signal centered at $1.53 \mathrm{ppm}$ (1), C-CDH-C. On removal of coupling between hydrogen and deuterium the latter one proton signal showed as an unresolved triplet.

$$
\text { 1,5-Anhydro-4-deoxy-L-xy10-hexitol-4,6,6- } \mathrm{H}_{3}^{2} \text { (c1s) (84) }
$$

$$
R_{F} 0.40 ;[\alpha]_{D}^{23}-45^{\circ} \text { (c, } 2.4 \text { in water). N.m.r. }
$$

signals $\left(\mathrm{D}_{2} \mathrm{O}\right)$; multiplet $3.0-4.15(5)$; unresolved signal. centered at $1.96 \mathrm{ppm}$ (1), C-CDH-C. On removal of coupling between hydrogen and deuterium the latter one proton signal was resolved into a quartet whose spacing was consistent with a pair of Iines separated by $5.1 \mathrm{c} / \mathrm{s}$ which 
were each split into two further lines $2.3 \mathrm{c} / \mathrm{s}$ apart.

$$
\frac{2,3,6-\operatorname{Tr} 1-0-\text { acety } 1-1,5 \text {-anhydro-4-deoxy-L-xylo- }}{\text { hexitol-4,6,6-H } 3 \text { (cis) }}
$$

The deuterated anhydrodeoxyhexitol (84) (37 mg) was. acetylated with acetic anhydride $(1 \mathrm{ml}$ ) and anhydrous pyridine ( $I \mathrm{mI})$. The product was isolated in the usual way and crystallised from ether-petroleum ether (b.p. 30-60 ), m.p. $82^{\circ} ;[\alpha]_{D}^{24}-43^{\circ}$ (c, 1.3 in chloroform). 


\section{Experimental Section B \\ 3,4,6-Tri-o-acetyl-D-galactal (40)}

(a) D-galactose (55 g) was added portionwise over 45 minutes, with control of temperature between $35-40^{\circ}$, to a stirred solution of acetic anhydride (200 ml) containing $70 \%$ perchloric acid $(1.2 \mathrm{ml})$, and the solution was stood overnight at room temperature, or at $40^{\circ}$ for 2 hours. Red phosphorus ( $15 \mathrm{~g}$ ) was added to the stirred solution, followed by the dropwise addition of bromine $(29 \mathrm{ml})$ and then of water ( $15 \mathrm{ml}$ ), the temperature being kept throughout at or below $20^{\circ}$ by cooling in an lce-water bath. The mixture was then warmed to room temperature and allowed to stand for 3 hours, then filtered with suction, the residue being washed with a little glacial acetic acid. The amber coloured solution containing 2,3,4,6-tetra-0-acetyl$\alpha$-D-galactopyranosyl bromide was immediately added dropwise to a zinc-acetic acid reduction mixture containing sodium acetate and copper sulphate, maintained at -5 to $-10^{\circ}$, prepared as described under 3,4-di-0-acetyl-D-xylal; subsequent reaction and isolation of crude 3,4,6-tri-o-acetylD-galactal was also carried out as described for the isolation of the pental. The crude product was purified by fractional distillation under high vacuum. The fraction b.p. $135-139 \% .5 \mathrm{~mm}(46 \mathrm{~g})$ crystallised slowly on standing in 
the refrigerator; $[\alpha]_{D}^{22}-15^{\circ}$ (c, 7.0 in chloroform): $n_{D}^{20}$ 1.4671; one zone by T.L.C. (benzene-methanol; $96: 4 \mathrm{v} / \mathrm{v}$ ). (b) $2,3,4,6$-Tetra-0-acetyl- $\alpha$-D-galactopyranosyl bromide (89) was 1solated during the preparation of 3,4,6-tri-0acetyl-D-galactal on one occasion. Chloroform (150 ml) was added to the hydrobromination reaction mixture after standing at room temperature for 2 hours, and the mixture was filtered through a layer of glass wocl, the reaction flask and fliter funnel belng washed with an additional $30 \mathrm{ml}$ of chloroform, and vigorously extracted with two portions ( $400 \mathrm{ml}$ and $150 \mathrm{ml}$ ) of 1ce-cold water. The chloroform layer was added to a stirred saturated aqueous solution of sodium hydrogen carbonate in a $I l$ beaker, and the mixture was transferred to a separatory funnel and thoroughly shaken. The separated chloroform layer was stirred for 10 minutes with dry silicic acid $(5 \mathrm{~g})$, filtered and evaporated under reduced pressure to a pale yellow syrup. This was dissolved in ether (300 $\mathrm{ml}$ ) and shaken with charcoal $(5 \mathrm{~g})$, calcium chloride $(5 \mathrm{~g})$ and sodium hydrogen earbonate $(0.5$ g), filtered, and the solvent removed under reduced pressure until about $75 \mathrm{ml}$ of ether remained. On standing in the refrigerator the product crystalized, and was recrystallis ed from ether-petroleum ether (b.p. 30-60 ). Yield $89 \mathrm{~g}$ of (89), m.p. $82-83^{\circ}$. 
In cases where attempted distillation of crude $3,4,6$ tri-O-acetyl-D-galactal under reduced pressure resulted in decomposition, purification was effected by chromatography on a column of Florisil, prewashed with anhydrous benzene, using benzene-methanol (100:2, v/v) as developing solvent. The first zone to be eluted (one spot by T.L.C.) was identified as 3,4,6-tri-0-acetyl-D-galactal by comparison of its infrared and n.m.r. spectra with those of an authentic sample.

Reaction of 3,4,6-Tri-o-acetyl-D-galactal with Carbon Monoxide and Hydrogen

Reaction conditions and product isolation procedures were in general similar to those employed previously with 3,4-di-o-acetyI-D-xylal. A solution of 3,4,6-tri-0-acetylD-galactal (15 g) and dicobalt octacarbonyl $(3.5 \mathrm{~g})$ in anhydrous benzene (50 $\mathrm{ml}$ ) was shaken with carbon monoxide (1300 psi) and hydrogen (1900 psi) in a high pressure reaction vessel at $130-135^{\circ}$ for $21 / 2$ hours. On cooling to room temperature the decrease in pressure was approximately equivalent to the absorption of 3 moles of gas $\left(\mathrm{CO}+2 \mathrm{H}_{2}\right)$. After pressure was released, the reaction mixture was transferred to a column (14 8 $8 \mathrm{~cm}$ diam.) of Florisil and catalyst was eluted with petroleum ether (b.p. 30-60\%). Elution with benzene-ethanol $(9: I, v / v)$ and evaporation of solvent gave a syrup $(13.5 \mathrm{~g})$. 
Deacetylation with $0.1 \mathrm{~N}$ sodium methoxide in methanol at room temperature for 18 hours, neutralisation with solid carbon dioxide and evaporation of solvent gave a watersoluble product which, after deionisation with Amberlite IR-120 $\left(\mathrm{H}^{+}\right)$resin and freeze-drying, afforded a partially crystalline product consisting principally of a mixture of two polyols in approximately equal amounts. Isolation of the two main components of the mixture was carried out by preparative paper chromatography to give Fraction $A\left(R_{F} 0.24\right)$ and Fraction $B\left(R_{F} 0.21\right)$. From an amount of $0.43 \mathrm{~g}$ of crude mixture, $0.16 \mathrm{~g}$ of $\mathrm{A}$ and $0.14 \mathrm{~g}$ of $\mathrm{B}$ were isolated.

\section{Characterisation of Fractions $A$ and $B$}

Fraction A (2,6-anhydro-3-deoxy-D-galacto-heptitol (91))

Fraction $A, R_{F} 0.24$, was dissolved in methanol, clarified and decolourised by filtration through a layer of Celite-Darco 60, and crystallised by the addition of isopropyl ether to turbidity; m.p. 158-159 ; $[\propto]_{\mathrm{D}}^{27}+24^{\circ}$ (c, 0.8 in water). Calc. for $\mathrm{C}_{7} \mathrm{H}_{14} \mathrm{O}_{5}: \mathrm{C}, 47.18, \mathrm{H}, 7.92$. Found: $\mathrm{C}, 47.50 ; \mathrm{H}, 8.07$. N.m.r. signals $\left(\mathrm{D}_{2} \mathrm{O}\right)$ : multiplet $3.25-4.13(8)$; multiplet $1.32-1.87 \mathrm{ppm}$ (2). 
2,6-Anhydro-3-deoxy-1, 4,5,7-tetra-0-p-nitrobenzoyl-

D-galacto-heptitol

Fraction A (35 mg) was heated with p-nitrobenzoyl chloride $(0.30 \mathrm{~g})$ in anhydrous pyridine $(1.5 \mathrm{ml})$ at $90^{\circ}$ for 1 hour. Isolation of the product in the usual way gave a sclid ( $95 \mathrm{mg}$ ) which was recrystallised from ethyl acetatepetroleum ether (b.p. $\left.30-60^{\circ}\right) ;$ m.p. 210-211 $1^{\circ}[\alpha]_{D}^{23}-12^{\circ}$

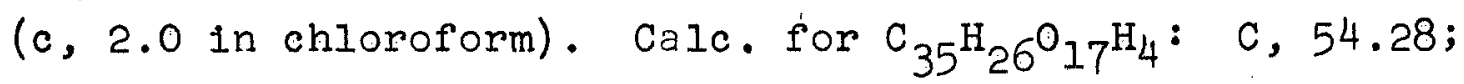
$\mathrm{H}, 3.38 ; \mathrm{N}, 7.23$. Found: C, $54.61 ; \mathrm{H}, 3.50 ; \mathrm{N}, 7.47$.

\section{Fraction B $(2,6$-anhydro-3-deoxy-D-talo-heptitol (90))}

The slower-moving component, $R_{F} 0.21$, was crystallised In a similar way from methanol-isopropyl ether; m.p. 168 ; $[\alpha]_{D}^{27}+68^{\circ}$ (c, 1.1 in water). Calc. for $\mathrm{C}_{7} \mathrm{H}_{14} \mathrm{O}_{5}: \mathrm{c}$, 47.18; H, 7.92. Found: C, 46.94; H, 8.22. N.m.r. signals $\left(D_{2} 0\right)$ : multiplet $3.36-4.33$ (8); multiplet $1.53-2.13$ ppm (2) .

$$
\begin{aligned}
& \text { 2,6-Anhydro-3-deoxy-1,4,5,7-tetra-0- }- \text {-n1trobenzoyl- } \\
& \stackrel{\text { D-talo-hept1t } \odot 1}{\underline{\underline{-}}}
\end{aligned}
$$

A portion of the crystalline Fraction $B$ was converted to a tetra-0-p-nitrobenzoyl derivative in the usual way to give a solid which was recrystallis ed from chloroform-

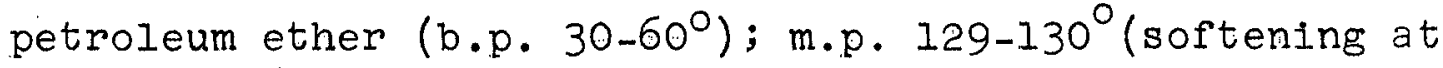


$\left.113-115^{\circ}\right) ; \quad[\alpha]_{D}^{23}+23^{\circ}$ (c, 0.8 in chloroform). Calc. for $\mathrm{C}_{35} \mathrm{H}_{26} \mathrm{O}_{17^{\mathrm{N}}}: \mathrm{C}, 54.28 ; \mathrm{H}, 3.38 ; \mathrm{N}, 7.23$. Found: C, 54.59; $\mathrm{H}, 3.45 ; \mathrm{N}, 7.70$.

2,6-Anhydro-3-deoxy-4,5-0-isopropylidene-D-talo-
heptitol (100)

Fraction $B(55 \mathrm{mg})$ in anhydrous acetone $(2 \mathrm{ml})$ containing $4 \%$ sulphuric acid was stirred at room temperature for 20 hours with exclusion of molsture. Neutralisation with $5 \mathrm{~N}$ sodium hydroxide solution, filtration to remove sodium sulphate and evaporation gave an oil. This was exhaustively extracted with bolling carbon tetrachloride, which on evaporation under reduced pressure gave a crystalIine residue of the mono-isopropylidene derivative (100) (23 $\mathrm{mg})$. Further treatment of the CCl with acidified acetone as described above afforded an additional amount (2l $\mathrm{mg}$ ) of the derivative. The combined products were recrystallis ad from carbon tetrachloride; m.p. $104-105^{\circ} ;[\propto]_{\mathrm{D}}^{21}+12^{\circ}$ (c, 1.6 in chloroform). Calc. for $\mathrm{C}_{10} \mathrm{H}_{18} \mathrm{O}_{5}: \mathrm{C}, 55.03 ; \mathrm{H}, 8.31$. Found: $\mathrm{C}, 54.82 ; \mathrm{H}$, 7.97 . 
2,6-Anhydro-3-deoxy-4,5-0-isopropylidene-1,7-di-0-

(p-tolylsulphonyl) -D-talo-heptitol (106)

To a solution of the mono-1sopropylidene derivative (100) (33 mg) in dry pyridine $(1.0 \mathrm{ml}$ ) was added p-toluenesulphonyl chlcride $(66 \mathrm{mg})$. After standing for 18 hours at room temperature in a stoppered flask the solution was stirred with water $(0.1 \mathrm{ml})$ for 20 minutes. More water was added until the solution became turbid, and on standing crystals $(50 \mathrm{mg})$ of the di----tolylsulphonyl derivative formed. The product was recrystallis ed from methanol; m.p. $135^{\circ} ; \quad[\alpha]_{D}^{23}-42^{\circ}$ (c, 1.7 in chloroform). Calc. for $\mathrm{C}_{24} \mathrm{H}_{30} \mathrm{O}_{9} \mathrm{~S}_{2}: \mathrm{C}, 54.78 ; \mathrm{H}, 5.74$. Found: $\mathrm{c}, 55.08 ; \mathrm{H}, 6.01$.

\section{Reaction of (106) with Sodium Iodide}

(a) When a solution of the di-0-p-tolylsulphonyl derivative (106) (8.4 mg) and sodium lodide (25 $\mathrm{mg})$ in acetone $(0.4 \mathrm{ml})$ was heated in a sealed tube at $118^{\circ}$ for 26 hours, sodium $\underline{p}$-toluenesulphonate was precipitated in an amount $(6.4 \mathrm{mg})$ equivalent to the replacement of both tosyloxy groups of (106) by 1odide.

(b) When the sealed tube reaction $(8.0 \mathrm{mg}$ of (106) in acetore $(0.5 \mathrm{ml})$ containing sodium iodide $(23 \mathrm{mg})$ ) was carried out at $100^{\circ}$, an appreciable quantity of sodium $\underline{p}-$ toluenesulphonate was precipitated within minutes. After 25 minutes the amount isolated $(2.8 \mathrm{mg}$ ) was approximately 
equivalent to the replacement of only one tosyloxy group of $(106)$.

\section{Consumption of Periodate Ion}

\section{Fraction $A$}

Absorbance readings at $223 \mathrm{~m} \mu$ were measured at intervals (Beckmann DU, $1 \mathrm{~cm}$ cells) of an aqueous solution containing $0.457 \times 10^{-4} \mathrm{M}$ of Fraction $\mathrm{A}$ and $0.917 \times 10^{-4} \mathrm{M}$ of sodium periodate (Reading A). Simultaneous readings were taken of a solution containing $0.457 \times 10^{-4} \mathrm{M}$ of Fraction $A(B)$, and of a solution containing $0.917 \times 10^{-4} \mathrm{M}$ of sodium periodate (C).

\begin{tabular}{l|c|c|c} 
TIme & $(B+C)-A(a)$ & $\frac{(B+C)-A}{C}(b)$ & $\begin{array}{l}\text { Moles periodate/ } \\
\text { mole substrate }\end{array}$ \\
\hline $1 \min$ & 0.289 & 0.312 & 0.63 \\
$47 \min$ & 0.448 & 0.483 & 0.97 \\
17 hours & 0.401 & 0.433 & 0.87
\end{tabular}

(a): decrease in absorbance due to consumption of periodate

(b): fraction of known amount of periodate consumed

\section{Fraction B}

By a similar procedure, but at a 15-fold dilution, corresponding values obta1ned with Fraction B were 


\begin{tabular}{l|c|c|c} 
Time (hrs) & $(B+C)-A$ & $\frac{(B+C)-A}{C_{0}}$ & $\begin{array}{c}\text { Moles periodate/ } \\
\text { mole substrate }\end{array}$ \\
\hline 1 & 0.463 & 0.473 & 0.88 \\
2 & 0.477 & 0.487 & 0.91 \\
9 & 0.478 & 0.490 & 0.92 \\
23 & 0.503 & 0.511 & 0.95
\end{tabular}

Enantiomeric 2-Deoxy-3--o-(1,3-d1hydroxy-2-propyl)glycero-tetritols (94) and (95)

Fraction A (53 mg) was dissolved in a solution (5 $\mathrm{ml}$ ) containing periodic acid ( $80 \mathrm{mg}, 50 \%$ excess), and the course of the oxidation was followed by observing the change in optical rotation of the solution, contained in a $2 \mathrm{dm}$ polarimeter tube protected from light. After 2 hours the solution was neutralised with barium carbonate and flitered into a solution of sodium borohydride (50 $\mathrm{mg}$ ) In water $(3 \mathrm{ml})$. After 90 minutes at room temperature the solution was neutralised with acetic acid, delonised by stirring with Amberlite IR-120 $\left(\mathrm{H}^{+}\right)$resin, filtered and evaporated to a solid, which was repeatedly evaporated with methanol to afford a syrup $(52 \mathrm{mg}) ;[\alpha]_{D}^{25}+21^{\circ}(c, 3.7$ in water): N.m.r. signals $\left(\mathrm{D}_{2} \mathrm{O}\right)$ : multiplet $3.50-3.85$, with sharp signal at 3.68 (10); multiplet (apparent quartet) $1.47-1.92 \mathrm{ppm}(2)$. 
The dextrorotatory tetrol ether was characterised as the tetra-0-p-nitrobenzoyl derivative; a portion (2I mg) was heated with $\underline{p}$-nitrobenzoyl chloride $(0.2 \mathrm{~g})$ in pyridine ( $1 \mathrm{ml}$ ) and the product was isolated in the usual manner as a syrup (85 mg) which solidifled slowly on standIng under methanol at room temperature, and was recrystallised from ethyl acetate; m.p. $150-151^{\circ} ; \quad[\alpha]_{D}^{24}+23^{\circ}$ (c, $0.9^{\prime}$ in chloroform). Calc. for $\mathrm{C}_{35} \mathrm{H}_{28} \mathrm{O}_{17} \mathrm{~N}_{4}$ : C, 54.13; H, 3.63; $\mathrm{N}, 7.21$. Found: C, 54.46; H, 3.55; N, 7.29.

Fraction B (49 mg) was similarly converted by periodate oxidation followed by sodium borohydride reduction, with isolation of the product as described above, to a levorotatory tetrol ether (49 mg); $[\alpha]_{D}^{25}-23^{\circ}$ (c, 3.2 in water), whose n.m.r. spectrum $\left(\mathrm{D}_{2} \mathrm{O}\right)$ was identical with that described above for the dextrorotatory enantiomer. A portion (2I mg) of the product was converted in the usual manner to a tetra-0-p-nitrobenzoyl derivative which was crystallized as described above; m.p. 150-1510; $[\alpha]_{D}^{24}-23^{\circ}$ (c, 1.1 in chloroform). Calc. for $\mathrm{C}_{35} \mathrm{H}_{28} \mathrm{O}_{17} \mathrm{~N}_{4}:$ C, 54.13 ; $\mathrm{H}, 3.63$. Found: C, 54.55; H, 3.71. The 1nfrared spectra of the two tetra-p-nitrobenzoates were identical. 
Attempted Synthese's of Optically Pure Tetrol Ethers

(a) Attempted Synthesis of L-1somer (95) from 2,5-Anhydro-

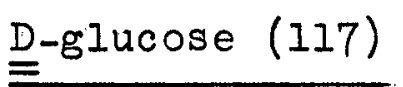

D-Arabino-tetraacetoxy-1-nitro-1-hexene (121)

To a suspension of D-arabinose $(25 \mathrm{~g})$ in anhydrous methanol (50 ml) and anhydrous nitromethane $(90 \mathrm{ml})$ in a $500 \mathrm{ml}, 3$-necked flask fitted with an efficient mechanical stirrer was added a solution of sodium $(5.25 \mathrm{~g})$ in anhydrous methanol (175 ml). The mixture was stirred at room temperature for 20 hours with exclusion of moisture, and the resulting solid mass of sodium ac1-nitroalcohols was collected by filtration and washed with a small volume of cold methanol and with petroleum ether (b.p. 30-60\%). The solid residue was immediately dissolved in ice-cold water (200 ml) and deionised by passage through a column containing Dowex$50\left(\mathrm{H}^{+}\right)$resin $(200 \mathrm{ml})$. The effluent and washings (total $500 \mathrm{ml}$ ) were concentrated under reduced pressure to a brown syrup, which was twice evaporated to dryness with absolute ethanol. On standing over $\mathrm{P}_{2} \mathrm{O}_{5}$ the residue solidified to a crystalline mass which was washed with cold ethanol to give a mixture of nitroalcohols (119) (16.5 g). I.R. (I1quid film): $1550 \mathrm{~cm}^{-1}$ (s), $1365 \mathrm{~cm}^{-1}$ (m) ( $\mathrm{NO}_{2}$ stretching). 
The mixture of nitroalcohols $(16.5 \mathrm{~g})$ was dissolved In acetic anhydride (200 ml) contalning 2 drops of concentrated sulphurle ac1d, and the solution was heated for 1 hour on a steam bath. Isolation of the product in the usual way gave a syrupy mixture of acetylated nitroalcohols (120). I.R. (I1quid f1Im): $1565 \mathrm{~cm}^{-1}$ (s), $1375 \mathrm{~cm}^{-1}$ (s) (NO 2 stretching). The product was dissolved in benzene $(600 \mathrm{ml})$, sodium hydrogen carbonate $(50 \mathrm{~g})$ was added and the mixture was refluxed for 2 hours. Filtration of the cooled mixture and removal of solvent under reduced pressure gave a pale yellow crystalline mass. Recrystallisation from ethanol gave D-arabino-tetraacetoxy-1-nitro-1-hexene (121), (16.5 g); m.p. 113-115 . I.R. (Nujol): $1510 \mathrm{~cm}^{-1}$ (m), $1350 \mathrm{~cm}^{-1}$ (m) (NO 2 in conjugation with $\mathrm{C}=\mathrm{C}$ ) $\because$

$$
\text { 2-Acetamido-1,2-d1deoxy-1-nitro-D-mann1tol (122) }
$$

To D-arabino-tetraacetoxy-1-nitro-1-hexene (15 g) in a $500 \mathrm{ml}$ filter flask was added methanol ( $150 \mathrm{ml}$ ); the mixture was cooled in ice and saturated with anhydrous ammonia, when the nitroolefin dissolved. After warming to room temperature over 8 hours with protection from molsture, the solvent was evaporated in a stream of dry nitrogen. The residue was filtered with the aid of cold absolute ethanol and recrystallised from ethanol to afford 2acetamido-1,2-dideoxy-1-nitro-D-mannitol (122) $(4.4 \mathrm{~g})$; 
m.p. $171-172^{\circ}$. A mixture of (122) and its epimer (123) was obtained from the mother liquors.

$$
\text { 2-Amino-2-deoxy-D-mannose hydrochloride (116) }
$$

A solution of (122) (4.4 g) in $2 \mathrm{~N}$ sodium hydroxide solution ( $10 \mathrm{ml}$ ) was added dropwise at room temperature to concentrated hydrochloric acid $(9 \mathrm{ml})$ with vigorous stirring. The resulting solution was brought briefly to boiling point, cooled to $0^{\circ}$, saturated with hydrogen chloride, and filtered to remove sodium chloride. After dilution with water (10 $\mathrm{ml})$ the solution was filtered through a layer of CeliteDarco 60 and concentrated under reduced pressure to a syrup, which was stood overnight under vacuum, over KOH, to remove residual hydrogen chloride. The product was crystallided by dissolving in methanol (10 ml) containing 2-3 drops of water and adding acetone to turbidity. Scratching, cooling and periodic additions of acetone afforded 2-amino-2-deoxy-D-mannose hydrochloride (3.2 g); $[\alpha]_{\mathrm{D}}^{22}-3.5^{\circ}(c, 4.3$ in water $)$.

Attempted Preparation of 2,5-Anhydrc-D-glucose (117)

To a solution of 2-amino-2-deoxy-D-mannose hydrochloride $(3 \mathrm{~g})$ in water $(50 \mathrm{ml})$ was added mercuric oxide $(16 \mathrm{~g})$ and the mixture was heated on a boling water bath for 30 minutes. The cooled mixture was filtered, saturated with hydrogen 
sulphide, refiltered through Celite-charcoal and evaporated under reduced pressure to a syrup. This did not crystalise on dissolving in a small volume of methanol; considerable darkenlng of the product ensued on standing and only a brown amorphous solid was obtained.

(b) Attempted Synthes1s of D-1somer (94) from 2,5-AnhydroD-mannose (115). 2,5-Anhydro-D-mannose (115)

A solution of 2-amino-2-deoxy-D-glucose hydrochloride $(15 \mathrm{~g})$ in water $(100 \mathrm{ml})$ was cooled in an 1ce-salt bath until a quantity of ice had formed in the solution. To this was added sodium nitrite $(6.0 \mathrm{~g})$ dissolved In ice-cold water (35 ml) containing glacial acetic acid ( $1 \mathrm{ml}) \because$ The resulting solution was kept near its freezing point for $2^{1 / 2}$ hours and then stood in the refrigerator for 24 hours. Glacial acetic acid $(1.5 \mathrm{ml})$ was added and the solution was vigorously aerated at room temperature for 30 minutes to remove nitrous acid, and then evaporated to a moblle syrup under reduced pressure at $25^{\circ}$. The product was dehydrated by several extractions w1th 10-15 ml portions of anhydrous acetone, dissolved in methanol, filtered and evaporated to a syrup, wh1ch was tw1ce evaporated to dryness under reduced pressure at $25^{\circ}$ with anhydrous benzene. The resulting 2,5-anhydro-D-mannose $(10.5 \mathrm{~g})$ was characterized as the p-nitrophenylhydrazone, m.p. 179-1810. 
Attempted Preparation of Acetylated Nitroolefin

To a stirred solution of 2,5-anhydro-D-mannose (10 g) in anhydrous methanol $(30 \mathrm{ml})$ and anhydrous nitromethane (40 ml) in a $250 \mathrm{ml}, 3$-necked flask was added a solution of sodium $(2.5 \mathrm{~g})$ in anhydrous methanol (70 ml), with immediate formation of a white solid. After stirring at room temperature for 3 hours with exclusion of moisture the solid was collected by filtration and washed with a small volume of cold methanol and withlopetroleum ether (b.p. $\left.30-60^{\circ}\right)$. The solid was dissolved in ice-cold water $(100 \mathrm{ml})$ and deionised by passage through a column of Dowex $-50\left(\mathrm{H}^{+}\right)$resin. Evaporation of the combined effluent and washings under reduced pressure gave a syrup which was dried by azeotroping with benzene-ethanol. The product $(7.5 \mathrm{~g})$ did not crystalise on standing over $\mathrm{P}_{2} \mathrm{O}_{5}$. I.R. (Iiquid film): $1550 \mathrm{~cm}^{-1}$ (s), $1365 \mathrm{~cm}^{-1}$ (m) (NO $\mathrm{N}_{2}$ stretchIng): T.L.C. (water-saturated ethyl methyl ketone); two closely-moving zones.

The mixture of nitro-alcohols $(7.5 \mathrm{~g})$ was dissolved In acetic anhydride (100 $\mathrm{ml}$ ) containing 2 drops of sulphuric acid and the solution was heated on a steam bath for $11 / 2$ hours and stood overnight at room temperature. Isolation of the product in the usual way gave a $\operatorname{syrup}(10.0 \mathrm{~g})$; I.R. (Ilquid film): $1565 \mathrm{~cm}^{-1}$ (s), $1375 \mathrm{~cm}^{-1}$ (s) (NO stretching). This was dissolved in benzene $(200 \mathrm{ml})$ and 


\section{$-179-$}

the solution was refluxed with sodium hydrogen carbonate $(20 \mathrm{~g})$ for 11 hours. The infrared spectrum of the syrupy product isolated by filtration and evaporation of solvent was unchanged from that of the mixture of acetylated nitroalcohols. No change in the infrared spectrum was observed on further refluxing in benzene solution with anhydrous sodium acetate.

2-Deoxy-3-0-(1,3-dihydroxy-2-propyl)-L-glycero-tetritol

(95) from 2,6-Anhydro-3-deoxy-D-gluco-heptitol (130) (a)

Oxidation of 2,6-anhydro-3-deoxy-D-gluco-heptitol (130) w1th an excess of periodic acid, followed by reduction of the resulting dialdehyde with an aqueous solution of sodium borohydride in water, and isolation of the product as described previously gave 2-deoxy-3-0-(1,3-dihydroxy-2propyl) -L-glycero-tetritol (95); $[\propto]_{D}^{22}+26^{\circ}$ (c, 2.9 in water). The tetrol ether (95) formed a tetra-o-p-nitrobenzoyl derivative, m.p. $151-152^{\circ} ;[\alpha]_{\mathbb{D}}^{21}+22^{\circ}$ (c, 1.2 in chloroform). The melting point of this derivative was undepressed on admixture with the corresponding derivative of the dextrorotatory tetrol ether prepared from Fraction $A$, $\left([\alpha]_{D}+23^{\circ}\right)$, and the infrared spectra of the two $\underline{p}-$ nitrobenzoates were 1dentical.

(a)

The structure of 2,6-anhydro-3-deoxy-D-gluco-heptitol(130) had been established by correlation with $=4,5,7-t r i-0-a c e t y 1-2,6-$

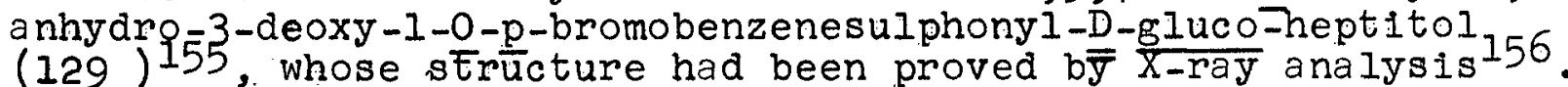




\section{Experimenta1 Section C}

Hydroformylation of $3,4-$ di-0-acetyl-D-xylal

3,4-Di-0-acetyI-D-xylaI (12.0 g) and dicobalt octacarbonyl ( $3 \mathrm{~g}$ ) were dissolved in anhydrous benzene; the volume of the solution $(60 \mathrm{ml})$, contained in the glass liner of a high pressure reaction vessel, gave a net void of $200 \mathrm{ml}$. After flushing with carbon monoxide, additional. carbon monoxide was added to a pressure of $600 \mathrm{psi}$, followed by hydrogen (2400 psi); on equilibration the initial gas pressure was 2930 psi at room temperature. The bomb was then heated with shaking to a temperature of $115^{\circ}$. The pressure increased to a maximum of 3730 psi after 35 minutes from the attainment of a constant temperature, and then began to fall. After an additional 30 minutes the reaction vessel and contents were rapidly cooled to room temperature by immersion in ice, when the pressure had decreased by 220 psi, to 2710 psi, equivalent to the absorption of 2 moles of synthesis gas $\left(\mathrm{H}_{2}+\mathrm{CO}\right)$. Unreacted gas pressure was released, the reaction mixture was transferred to a column of Florisil and catalyst was eluted with petroleum ether (b.p. $30-60^{\circ}$ ). Elution with benzene-isopropyl alcohol $(9: 1, v / v)$ and removal of solvent then gave a syrup $(10.0 \mathrm{~g})$. An additional quantity of a mobile syrup $(2.5 \mathrm{~g})$ was subsequently recovered by filtration and 
evaporation: of the petroleum ether fraction, after decomposition of catalyst on standing at room temperature. The latter fraction, identified as 3,4-di-0-acetyl-D-xylai (I.R. spectrum and T.L.C. (benzene-methanol, 96:4, v/v) alongside an authentic specimen) crystalilded on standing in the refrigerator.

T.I.C. of the main fraction (benzene-methanol, 95:5. $\mathrm{v} / \mathrm{v}$ ) alongside 3,4-di-0-acetyl-D-xylal and the mixture of di-O-acetyl-anhydrodeoxy-hexitols previously obtained by the hydroxymethylation of (22), showed two well separated zones corresponding to both controls. Chromatography of a portion $(1.5 \mathrm{~g})$ of the main fraction on a column of neutral alumina, using benzene-methanol $(95: 5, \mathrm{v} / \mathrm{v})$ as developing solvent, gave 3,4-di-0-acetyl-D-xylal (0.55.g) and a mixture of reaction products $(0.93 \mathrm{~g})$. The latter fraction showed a n.m.r. signal $\left(\mathrm{CCl}_{4}\right)$ at $\delta=9.35 \mathrm{ppm}$ (CHO) (intensity relative to acetate absorption around $\delta=2$ ppm consistent with approximately $15 \%$ di-0-acetyldeoxyantydro-aldehydo-hexoses (133) and (134)).

\section{Reaction with 2,4-Dinitrophenylhydrazine}

A portion $(1.2 \mathrm{~g})$ of the reaction mixture eluted from Florisil with benzene-isopropyl alcohol was dissolved in ethanol (20 ml) containing $2-3$ drops of acetic acid, and the solution was heated to bolling point on a steam 
bath. To this was added portionwise a hot, saturated solution of 2,4-dinitrophenylhydrazine tin ethanol, until the orange colour imparted to the solution no longer faded to yellow: On diluting with water to turbidity, and standing In the refrigerator, a yellow solid $(0.39 \mathrm{~g})$ separated, which was collected by filtration and dried over calcium chloride. T.I.C. (benzene-methanol, 95:5, v/v); 2 closely moving components, Fraction $X$ (faster-moving) and Fraction $Y$ (slower-moving), plus traces of others; no additional zones revealed with sulphuric-nitric acid spray reagent. N.m.r. signals $\left(\mathrm{CDCI}_{3}\right)$ : singlet 11.07 (1), N-NH-; doublet $(\mathrm{J}=3 \mathrm{c} / \mathrm{s}) 9.04(\mathrm{I})$, aromatic $\mathrm{H}_{3} ;$ quartet 8.30 (I), aromatic $\mathrm{H}_{5}$; pair of overlapping $\operatorname{doublets}(\mathrm{J}=9 \mathrm{c} / \mathrm{s}$ ), 7.87 and 7.95 ( 1 ), aromatic $\mathrm{H}_{6}$; doublet $(\mathrm{J}=6 \mathrm{c} / \mathrm{s}) 7.58$ (1), $\mathrm{N} \mathrm{CH}-$; multiplet $3.15-5.35$ (5); 3 sharp signals at $2.07,2.12$ and $2.16 \mathrm{ppm}(6), \mathrm{OCOCH}_{3}$, superimposed on multiplet (2), $\mathrm{C}-\mathrm{CH}_{2}-\mathrm{C}$.

Fraction $Y(4,5-D 1-0$-acetyl-2,6-anhydro-3-deoxy-
aldehydo-D-1yxo-hexose 2,4 -dinitrophenylhydrazone)

When the mixture of derivatives from the reaction with 2,4-dinitrophenylhydrazine was triturated with warm ethanol (2-3 ml), partial dissolution occurred and a granular yellow solid separated. This was isolated by filtration, washed with a little cold ethanol, and 
recrystallized as fine yellow needles from chloroformhexane; m.p. 225-226 ; $[\propto]_{\mathbb{D}}^{22}-60^{\circ}$ (c, 2.5 in chloroform). One spot by T.I.C. (benzene-methanol, 95:5, v/v) corresponding to slower-moving of two components present in mixture. N.m.r. $\left(\mathrm{CDCl}_{3}\right)$ : differed from mixture of $\mathrm{X}$ and $Y$ described above in showing doublet $(J=9 d / s)$ at 7.85 (1), aromatic C-6 proton, and two sharp signals 2.13 and $2.17 \mathrm{ppm}(6), \mathrm{OCOCH}_{3}$. Calc. for $\mathrm{C}_{16} \mathrm{H}_{18} \mathrm{~N}_{4} \mathrm{O}_{9}: \mathrm{C}, 46.83$; $\mathrm{H}, 4.42 ; \mathrm{N}, 13.66$. Found: C, $46.60 ; \mathrm{H}, 4.64 ; \mathrm{N}, 13.77$.

\section{1,5-Anhydro-4-deoxy-D-arabino-hexitol (44) from Fraction Y}

An additional amount $(0.9 \mathrm{~g})$ of Fraction. Y was prepared by reaction of the main fraction from the hydroformylation of (22) (6.8 g) with 2,4-dinitrophenylhydrazine, and isolation as described above. A portion $(0.4 \mathrm{~g})$ in chloroform ( $3 \mathrm{ml}$ ) was added to a mixture consisting of freshly distilled pyruvic acid $(4 \mathrm{ml})$ and a $10 \%$ solution of hydrogen bromide in acetic acid $(0.2 \mathrm{ml})$. The solution was heated at $40-50^{\circ}$ for $I$ hour and then stood overnight at rom temperature. A yellow crystalline solid (0.2 g) was isolated by filtration, washed with a small volume of cold chloroform, and identified as pyruvic acid 2,4-dinitrophenylhydrazone (m.p., I.R. spectrum). The filtrate was extracted with two $10 \mathrm{ml}$ portions of chloroform, and the extract was washed with three $10 \mathrm{ml}$ portions of saturated sodium hydrogen carbonate solution to remove dissolved pyruvic acid 2,4-dinitro- 
phenylhydrazone, and then with water. After drying over magnestum sulphate and filtering, solvent was removed under reduced pressure to afford a syrup (110 $\mathrm{mg}$ t recovery not quantitative). N.m.r. $\left(\mathrm{CCl}_{4}\right)$ : singlet at $9.35 \mathrm{ppm}(\mathrm{CHO})$. I.R. (Ilquid film): carbonyl bands at $1740 \mathrm{~cm}^{-1}$ (ester) and $1700 \mathrm{~cm}^{-1}$ (aldehyde); band at $3400 \mathrm{~cm}^{-1}$ (OH).

When a portion $(20 \mathrm{mg})$ of the aldehydo-compound was reacted in ethanol solution with 2,4-dinitrophenylhydrazine as described previously, a crystalline product separated on cooling which was identified as Fraction $Y$ (m.p., T.I.C., n.m.r.).

A portion ( $35 \mathrm{mg}$ ) of the aldehydo-product from the exchange reaction with pyruvic acid was dissolved in methanol ( $2 \mathrm{ml}$ ) and added dropwise to a solution of sodium borohydride $(30 \mathrm{mg})$ in water $(2 \mathrm{ml})$. After standing overnight at room temperature the solution was neutralised by the addition of acetic acid and deionised by passage through a small column of Amberlite $\mathrm{IR}-120\left(\mathrm{H}^{+}\right)$resin. The combined effluent and washings were evaporated to a solid residue which was repeatedly evaporated to dryness with methanol to afford a syrup ( $8 \mathrm{mg}$ ). One spot on paper. chromatography, alore and when superimposed on 1,5-anhydro4-deoxy-D-arabino-hexitol (44), two spots when superimposed on the slower-moving L-xylo-isomer (45). 
Fraction X (4,5-Di-O-acetyl-2,6-anhydro-3-deoxy-aldehydo-

D-xylo-hexose 2,4-dinitrophenylhydrazone)

The alcohol-soluble portion of the mixture of hydrazones remaining after 1solation of Fraction $Y$ as described above was evaporated to a syrup. A portion ( $45 \mathrm{mg}$ ) was applied in chloroform solution as a narrow streak near the lower edge of a plate $(20 \mathrm{~cm}$ wide $\times 45 \mathrm{~cm}$ long $\times 0.8 \mathrm{~mm}$ thick) of silica gel G, and fractionated by multiple ascending development, using chloroform as developing solvent, with air drying between successive developments. The faster-moving of the two major components was cut out, eluted with chloroformethanol $(1: 1, v / v)$, filtered and evaporated to a syrup which was crystallised from chloroform-hexane as fine needles; m.p. $132^{\circ} ;[\alpha]_{D}-16^{\circ}$ (c, 0.4 in chloroform). N.m.r. $\left(\mathrm{CDCl}_{3}\right)$ : one acetoxy signal at $2.05 \mathrm{ppm}$. Calc. for $\mathrm{C}_{16}{ }^{\mathrm{H}} 18^{\mathrm{N}} 4_{9}^{\mathrm{O}}: \mathrm{C}$, $46.83 ; \mathrm{H}, 4.42$. Found: C, 46.90; H, 4.10.

$\frac{\text { Di-0-acetyl-anhydrodeoxy-aldehydo-hexoses (133) and }}{(134) \text { by oxidation of Di-0-acetyl-anhydrodeoxyhexitols }}$
$(74)$ and $(75)$

(a) To a portion $(0.88, g)$ of the syrupy product from the hydroxymethylation of 3,4-di-o-acetyl-D-xylal (section A), in anhydrous pyridine $(5 \mathrm{ml})$ was added p-toluenesulphonyl chlorlde $(1.0 \mathrm{~g})$, and the mixture was stood at room temperature 
for 20 hours. Isolation of the product in the usual way gave a syrup ( $1.0 \mathrm{~g}$ ) containing the 6-0-p-tolysulphonyl derivatives (76) and (77). I.R. (Iiquid film); bands at $1180 \mathrm{~cm}^{-1}$ ( $S=0$ stretching) and $1600 \mathrm{~cm}^{-1}$ (aromatic); no hydroxyl absorption. The product $(1.0 \mathrm{~g})$ in anhydrous dimethylsulphoxide $(5 \mathrm{ml})$, was added dropwise to a mixture of dimethylsulphoxide $(20 \mathrm{ml})$ and sodium hydrogen carbonate $(3 \mathrm{~g})$, through which was passing a stream of nitrogen, and which was maintalned at $150^{\circ}$ in an oil bath. After 5 minutes the mixture was cooled, filtered, and solvent was removed under reduced pressure to afford a brown syrup, whose infrared spectrum was unchanged from that described above.

(b) To a solution of the hydroxymetrylation product comprising di-O-acetyl-anhydrodeoxyhexitols (74) and (75) $(0.37 \mathrm{~g})$ in anhydrous dimethylsulphoxide $(6 \mathrm{ml})$, was added anhydrous phosphorlc acid $(0.1 \mathrm{ml})$ and $N, N^{\prime}$-dicyclohexylcarbodilmide $(1.8 \mathrm{~g})$. After standing at room temperature under anhydrous conditions for 24 hours the mixture was filtered, and the crystalline residue of $N_{,} N^{\prime}$-dicyclohexylurea was washed with absolute ethanol to give a total volume (filtrate + washings) of $30 \mathrm{ml}$. To a portion $(15 \mathrm{ml}$ ) of this solution, heated on a steam bath, was added a hot, saturated solution of 2,4-dinitrophenylhydrazine in ethanol, until an orange colour persisted on boiling. Dilution with water to 
turbldity and cooling gave a yellow solld which was collected by filtration and dried. Separation of 2,4-dinitrophenylhydrazones from co-precipitated $\mathrm{N}_{2} \mathrm{~N}^{+}$-dicyclohexylurea was effected by extraction of the former with a small volume of cold chloroform, filtration and evaporation to a yellow solid (165 mg). Preparative scale TLC of a portion (30 mg) of the mixture of hydrazones afforded two pure components; 4,5-di-o-acety1-2,6-anhydro-3-deoxy-aldehydo-D-1yxo-hexose 2,4-dinitrophenylhydrazone (17 mg), m.p."225-226 , and the 2,4-dinitrophenylhydrazone of the corresponding D-xyloisomer, (9 mg), m.p. $132^{\circ}$, both identified with the two fractions ( $Y$ and $X$ respectively) described previousiy.

When the same experiment was carried out with omitssion of the mixture of acetylated anhydrodeoxyhexitols, no precipitate was isolated following dilution of the reaction mixture with water. Similarly, a control experiment in which $N_{,} N^{\prime}$-dicyclohexylcarbodilmide was not present did not result in the isolation of any reaction product following treatment with 2,4-dinitrophenylhydrazine. 


\section{REFERENCES}

1. H. Akins and G. Krsek, J. Am. Chem. Soc., 70, 383 (1948).

2. I. Wender, M. Orchin and H. H. Storch, J. Am. Chem. Soc. 72, 4842 (1950).

3. F. Flscher and H. Tropsch, Brennstoff-Chem., 4, 276 (1923).

4. D. F. Smith, C. O. Hawk and P. L. Golden, J. Am. Chem. Soc., 52, 3221 (1930).

5. 0. Roelen, Ger. Patent 103,362 (f1led 1938): U.S. Patent 2,327,066 (1943): Chem. Abstracts, 38, 550 (1944).

6. I. Wender, H. W. Sternberg and M. Orchin, "Catalysis", Vol. 5, ed. by P. H. Emmett, New York, Reinhold Publishing Corporation, 1957, p. 73.

7. H. W. Sternberg and I. Wender, Proceedings of International Conference on Coordination Chemlstry, Chem. Soc. Spec. Publn. No. 13, (1959), p. 35 .

8. C.W. Bird, Chem. Rev., 62, 283 (1962).

9. H. Adkins and G. Krsek, J. Am. Chem. Soc., 7.1, 3051 (1949).

10. J. Halpern, Quart. Rev., 10, 463 (1956).

11. M. Orchin, L. Kirch and I. Goldfarb, J.Am. Chem. Soc., 78, 5450 (1956).

12. A. R. Martin, Chem. and Ind., 1536 (1954).

13. G. Natta, R. Ercol1, S. Castellano and P. H. Barbleri, J. Am. Chem. Soc., 7:6, 4049 (1954).

14. G. L. Karapinka and M. Orchin, J. Org. Chem., 26, 4187 (1961).

15. I. Kirch and M. Orchin, J.Am. Chem. Soc., 80, 4428 (1958).

16. I. Wender, S. Metlin, S. Ergun, H. W. Sternberg and H. Greenfield, J. Am. Chem. Soc., 78, 5401 (1956).

17. R. F. Heck and D. S. Breslow, J.Am. Chem. Soc., 83, 
18. D. S. Breslow and R. F. Heck, Chem. and Ind., 467 (1960).

19. T. H. Coffleld, J. Kozikowski and R. D. Closson, J. Org. Chem., 22, 598 (1957).

20. L. Marko, Proc. Chem. Soc., 67 (1962).

21. C. L. Aldridge and H. B. Jonassen, Nature, 188, 404 (1960).

22. C. L. Aldridge and H. B. Jonassen, J. Am. Chem. Soc., 85,

23. J. G. Traynham, J. Am. Chem. Soc., 78, 4042 (1956).

24. I. Wender, J. Feldman, S. Metlin, B. H. Gwynn and M. Orchin, J. Am. Chem. Soc., 77, 5760 (1955).

25. J. Falbe and F. Korte, Ber., 97, 1104 (1964).

26. I. Wender, R. Levin and M. Orchin, J. Am. Chem. Soc., 72,
$4375(1950)$.

27. E. I. du Pont de Nemours and Co., Brit. Patent 614,010; Chem. Abstracts, 43, 4685 (1949).

28. C.W. Bird, R. C. Cookson, J. Hudec and R. O. Williams, J. Chem. Soc., 410 (1963).

29. P. F. Beal, M. A. Rebenstorf and J. E. Pike, J. Am. Chem. Soc., 81, 1231 (1959).

30. A. I. Nussbaum, T. I. Popper, E. P. Oliveto, S. Friedman and I. Wender, J. Am. Chem. Soc., 81, 1228 (1959).

31. E. FIscher and K. Zach, Sitz. ber. kgl. preuss. Akad. W1ss., 16, 311 (1913); Chem. Abstracts, 8, 73 (1914).

32. R. K. Ness and H. G. Fletcher, J. Org. Chem., 28, 435

33. E. F1scher, Eer., 47, 196 (1914).

34. E. Fischer, M. Bergmann and H. Schotte, Ber., 53, 509
$(1920)$.

35. L. D. HaII and L. F. Johnson, Tetrahedron Letters, 20 ,

36. M. Karplus, J. Chem. Phys., 30, 11 (1959). 
37. B. Helferich, Advances in Carbohydrate Chem., 7, 209 (1952).

38. 38. D. A. Prins and R. W. Jeanloz, Ann. Rev. Blochem., 17,

39. W. G. Overend and M. Stacey, Advances in Carbohydrate Chem., 45 (1953).

40. M. Barczai-Martos and F. Körösky, Nature, 165, 369 (1950).

41. R. E. Deriaz, W. G. Overend, M. Stacey, E. G. Teece and L. F. Wiggins, J. Chem. Soc., 1879 (1949).

42. W. G. Overend, F. Shafizadeh and M. Stacey, J. Chem. Soc., 671 (1950).

43. B. M. Iselin and T. Reichstein, Helv. Chim. Acta, 27, 1146,1200 (1944).

44. B. Helferich, E. N. Mulcahy and H. Ziegler, Ber., 87 ,

45. B. Helferich and M. Gindy, Ber., 87, 1488 (1954).

46. L. F. Fleser and M. Fiser, Advanced Organic Chemistry, Reinhold Publishing Corp., New York, N.Y., 1961, p. 310.

47. H. S. Isbell and W.W. P1gman, J. Research NatI. Bur. Standards, 22, 397 (1939).

48. W. G. Overend, F. Shafizadeh and M. Stacey, J. Chem. Soc., 992 (1951).

49. F. B. Cramer, J. Franklin Inst., 253, 277 (1952).

50. C. K. Ingold, Structure and Mechanism in Organic Chemistry, Cornell University Press, Ithaca, N.Y., 1953, p. 669.

51. J. Melnwald, Y. C. Melnwald and T. N. Baker, J. Am. Chem. Soc., 85, 2513 (1963).

52. W. J. Serfontein, J.H. Jordaan and J. White, Tetrahedron Letters, 18, 1069 (1964).

53. A. Schonberg and A. Mustafa, Chem. Rev., 40, 181 (1947).

54. B. Helferich and E. von Gross, Ber., 85, 531 (1952).

55. R. C. Hockett, A. C. Sapp and S. R. Millman, J. Am. Chem. Soc., 63, 2051 (1941). 


\section{$-\min 4$}

56. D. H. R. Barton and R. C. Cookson, Quart. Rev., 10, 44 (1956).

57. J. Chatt, Chem. Rev., 48, 15 (1951).

58. P. T. Manolopoulos, M. Mednick and N. N. Lichtin, J. Am. Chem. Soc., 84, 2203 (1962). 59. P. A. Levene and A. L. Raymond, J. B10l. Chem., 88, 513
(1930).

60. R. U. Lemieux and B. Fraser-Reid, Can. J. Chem., 42,

61. R. U. Lemieux and S. Levine, Can. J. Chem., 40, 1926
(1962).

62. A. Rosentha 1, D. Read and C. Cameron, Sclence, 123, 1177 (1956).

63. A. Rosenthal and D. Read, Can. J. Chem., 35, 788 (1957).

64. A. Rosenthal and D. Read, Methods in Carbohydrate Chemistry, Vol. II, ed. by R. L. Whistler and M. L. Wolfrom, New York, Academic Press, 1963, p. 457.

65. P. A. Levene and T. Mori, J. B1ol. Chem., 83, 803 (1929).

66. F. Weygand, Methods in Carbohydrate Chemistry, Vol. I, ed. by R. L. Whistler and M. L. Wolfrom, New York, Academic Press, 1962, p. 182.

67. W. G. Overend, F. Shaf1zadeh and M Stacey, J. Chem. Soc., 1027 (1950).

68. A. M. Gakhokidze, Zhur. Obsche1 Kh1m., 15, 530 (1945); Chem. Abstracts, $\stackrel{40}{=} 4673$ (1946).

69. D. W. Read, B.A. Thesis, University of British Columbia, 1954.

70. I. Wender, Ph.D. Thesis, University of P1ttsburgh, 1950.

71. C. T. Bishop, Methods Biochem. Anal. 10, I (1962).

72. G. Zemp1en, A. Gerecs and I. Hadacsy, Ber., 69, 1827

73. J. Baddiley, J. G. Buchanan, R. E. Handschumacher and J. F. Prescott, J. Chem. Soc., 2818 (1956). 
74.: J. M. Bobbit, Advances in Carbohydrate Chem., 1.1, I (1956).

75. R. D. Guthrie, Methods in Carbohydrate Chem1stry, Vol. I, ed." by R. L. Whistler and M. L. WoIfrom, New York, Academic Press, 1962, p. 435.

76. J. S. Dixon and D. Lipkin, Anal. Chem., 26, 1092 (1954).

77. C. E. Crouthamel, H. V. Meek, D. S. Martin and C. V. Banks, J. Am. Chem. Soc., 71, 3031 (1949). 78. Rules of Carbohydrate Nomenclature, J. Org. Chem., 28,
281 (1963).

79. I. M. Jackman, Applications of Nuclear Magnetic Resonance Spectroscopy in Organic ChemIstry, Pergamon Press Ltd., London, 1959, p. 55 .

80. E. von Rudloff, D. E. Stuetz and H. F. Bauer, Can. J. Chem., 35, 315 (1957).

81. M. L. Wolfrom and A. Thompson, Methods in Carbohydrate Chemistry, Vol. II, ed. by R. L. Whistler and M. L. Wolfram, New York, Academ1c Press, 1963, p. 65.

82. P. A. J. Gorin, Can. J. Chem., 38, 641 (1960).

83. F. L. Benton and T. E. Dilion, J.Am. Chem. Soc., 64, 1128 (1942).

84. Our thanks are due to Dr. E. von Rudloff, National Research Council Prairie Regipnal Laboratory, Saskatoon, Sask., for supplying a sample of this compound.

85: P. A. J. Gorin, J. Org. Chem., 24, 49 (1959).

86. A. S. Perlin, E. von Rudloff and A. P. Tulloch, Can. J. Chem., 35, 1504 (1957).

87. A. B. Foster, W. G. Overend, M. Stacey and G. Vaughan, J. Chem. Soc., 3367 (1954).

88. F. Shafizadeh, Methods in Carbohydrate Chemistry, Vol. II, ed. by R. I. Whistier and M. Wolfrom, New York, Academic Press, 1963, p. 409.

89. F. Shafizadeh and M. Stacey, J. Chem. Soc., 3608 (1952).

90. C. Tamm and T. Reichstein, Helv. Chim. Acta, 31, 1630 (1948). 
91. A. B. Foster, W. G. Overend and M. Stacey, J. Chem. Soc.,
974 (195I).

92. W. N. Haworth, L. N. Owen and F. Smith, J. Chem. Socz, 88 (1941).

93. P. A. Levene and A. I. Raymond, J. B101. Chem., 102, 317 (1933).

94. J. W. H. Oldham and J. K. Rutherford, J. Am. Chem. Soc., 54, 366 (1932).

95. S. Peat, Advances in Carbohydrate Chem., 2, 38 (1946).

96. R. Criegee, E. Buchner and W. Walther, Ber., 73, 571 (1940).

97. C. S. Hudson, J. Am. Chem. Soc., 31., 66 (1909).

98. B. Coxon and H. G. Fletcher, J. Am. Chem. Soc., 85, 2641 (1963).

99. C.W. Smith (to Shell Development Co.), U.S. Patent 2,489,729 (1949); Chem. Abstracts, 44, 30366 (1950).

100. K. Zlegler, Organ1c Synth., Col. Vo1. I, 314 (1941).

101. V. Migrdichian, The Chemistry of Organic Cyanogen Compounds, Reinhold Publishing Corp., New York, 1947, p. 219.

102. B. A. Nelson, E. J. Hodges and J. I. Simon, J. Org.Chem., 21, 798 (1956).

103. R. Zelinski and K. Yorka, J. Org. Chem., 23, 640 (1958).

104. I. J. Bellamy, The Infrared Spectra of Complex Molecules, Methuen and Co. Ltd., London, 1958, p. 225.

105. J. Davoll, J. Chem. Soc., 2526 (1949).

106. R. Paul, Compt. rend., 198, 375 (1934).

107. R. P. Zelinsk1, N. G. Peterson and H. R. Wallner, J. Am. Chem. Soc., 74, 1504 (1952).

108. W. A. Bonner, Advances in Carbohydrate Chem., 6, 251 (1951): Methods in Carbohydrate Chemistry, VVi. II, ed. by R. L. Whistler and M. L. Wolfrom, New York, Academic Press, 1963, p. 465.

109. J. Houben and K. Fuhrer, Ber., 40, 4990 (1907). 
110. C. Paal and F. Hornste1n, Ber., 39, 1361 (1906).

111. E. F1scher and K. Hess, Ber., 45, 912 (1912).

112. C. G. Jeremias and C. A. Mackenzie, J. Am. Chem. Soc., 70, 3920 (1948).

113. C. D. Hurd and W. A. Bonner, J. Am. Chem. Soc., 67,
ig72 (1945).

114. W. A. Bonner, J. Am. Chem. Soc., 68, 1711 (1946).

115. C. D: Hurd and W. A. Bonner, J. Am. Chem. Soc., 67,
i664

116. J. Yosh1mura, N. Maramatsu and T. Sato, Nippon Kagaka Zassh1, 79, 1503 (1958). 117. E. V1scher and T. Reichstein, Helv. Chim. Acta, 27,
i332 (1944).

118. K. Freudenberg and K. Rasch18, Ber., 60, 1633 (1927).

119. R. C. Hockett and M. I. Downing, J. Am. Chem. Soc., 64. 2463 (1942).

120. H. Ohle and E. Dickhauser, Ber., 58, 2593 (1925).

121. K. Gatzi and T. Reichstein, Helv. Ch1m. Acta, 21, 914 (1938).

122. A. Rosenthal and H. J. Koch, Can. J. Chem., 42, 2025 (1964).

123. R. U. Lemieux, R. K. Kullnig, H. J. Bernstein and W. G. Schneider, J. Am. Chem. Soc., 80, 6098 (1958).

124. M. Karplus, J. Am. Chem. Soc., 85, 2870 (1963).

125. J. A. Pople, W. G. Schelder and H. J. Bernstein, High Resolution Magnet1c Resonance, McGraw-H1li Book Company, Inc., New York, 1959, p. 132.

126. P. K. W. Woo, H. W. Dion and L. F. Johnson, J. Am. Chem. Soc., 84, 1066 (1962).

127. L. D. Hall, Advances in Carbohydrate Chem., 19, in press.

128. R. U. Lemieux and J. Howard, Can. J. Chem., 41, 393 (1963).

129. R. U. Lemieux and S. Levine, Can. J. Chem., 42, 1473 (1964). 
130. R. U. Lemseux and J.W. Lown, Can. J. Chem., 42, 893 (1964).

131. R. J. Abraham, R. Freeman, L. D. Hall and K. A. McLauchlan, J. Chem. Soc., 2080 (1962).

132. R. A. Edington, E. L. Hirst and E. E. Percival, J. Chem. Soc., 2281 (1955). 133. P.A. Levene and R. S. Tipson, J. Biol. Chem., 93, 631
(1931).

134. F. J. Bates and Associates, "Polarimetry, Sacchar metry and the Sugars", Nat. Bur. Standard C1rcular C440 (1942), p. 532 .

135. R. Criegee, L. Kraft and B. Rank, Ann., 507, 159 (1933).

136. J. W. Pratt; N: K. R1chtmyer and C. S. Hudson, J.Am. Chem. Soc., 74, 2200 (1952).

137. J. Honeyman and C. J. G. Shaw, J. Chem. Soc., 2454 (1959).

138. S. J. Angyal and C. G. Macdonald, J. Chem. Soc., 686 (1952).

139. R. J. Abraham, L. D. Hall, L. Hough and K. A. McLauchlan, Chem. and Ind., 213 (1962); J. Chem. Soc., 3699 (1962).

140. R. U. Lemièux, J. D. Stevens and R. R. Fraser, Can. J. Chem., $\stackrel{40}{=}, 1955$ (1962).

141. A. B. Foster, W. G. Overend, M. Stacey and L. F. Wiggins, J. Chem. Soc., 2542 (1949).

142. J. A. Mills, Advances in Carbohydrate Chem., 10, I (1955).

143. A. T. Ness, R. M. Hann and C. S. Hudson, J. Am. Chem. Soc., 66, 1901 (1944):

144. R. M. Hann and C. S. Hudson, J. Am. Chem. Soc., 67, 602

145. W. T. Haskins, R. M. Hann and C. S. Hudson, J. Am. Chem. Soc.: 65, 67 (1943).

146. J. C. Sowden, Advances in Carbohydrate Chem., 6, 291
(1951).

147. H. Ledderhose, Z. phys1ol. Chem., $\stackrel{4}{=} 139$ (1880). 


$$
-196-
$$

148. B. C. Bera, A. B. Foster and M. Stacey, J. Chem. Soc., 4531 (1956).

149. S. Akiza and T. Osawa, J. Pharm. Soc. Japan, 74, 1259

150. P. A. Levene and F. B. LaForge, J. B101. Chem., 2I,
345,351 (1915).

151. P. A. Levene, J. Biol. Chem., 39, 69, (1919).

152. J. C. Sowden and M. T. Oftedahl, Methods in Carbohydrate Chemistry, Vol. I, ed. by R. L. Whistler and M. L. Wolfrom, New York, Academic Press, 1962, p. 235.

153. A. B. Grant, New Zealand J. Sc1. Technol., 37, 509 (1956).

154. Reference 104, p. 299.

155. A. Rosenthal and H. J. Koch, unpublished results.

156. A. Camerman and J. Trotter, Acta Cryst., in press.

157. M. Somogy1, J. B101. Chem., 160, 69 (1945).

158. R. E. J. Mitchel, B.Sc. Thesis, University of British Columbia (1963).

159. Reference 79, p. 62 .

160. R. L. Shriner, R. C. Fuson and D. Y. Curting Systematic Identification of Organic Compounds, 4 th Ed. New York, John Wiley and Sons, 1956, p. 98.

161. D. Y. Curtin, J. A. Gourse; W. A. Richardson and K. I. Rinehart, J. Org. Chem., 24, 93 (1959).

162. V. R. Mattox and E. C. Kendall, J.Am. Chem. Soc., 70, 882 (1948); J. Biol. Chem., 188, 287 (1951).

163. M. L. Wolfrom, J. Am. Chem. Soc., 53, 2275 (1931).

164. M. I. Wolfrom and W. M. Morgan, J. Am. Chem. Soc., 54, 3390 (1932).

165. N. Kornblum; W. J. Jones and G. J. Anderson, J. Am. Chem. Soc., 81, 4113 (1959).

166. K. E. Pfitzner and J. G. Moffatt, J. Am. Chem. Soc., 85, (1963). 


$$
-157-
$$

167. D. H. R. Barton, B. J.Garner and R. H. Wightman, J. Chem. Soc., 1855 (1964).

168. G. Henseke and G. Hanisch, Angew. Chemie, 75, 420 (1963). 